GEAP- 10542

AEC RESEARCH AND

DEVELOPMENT REPORT

MARCH 1972

\title{
FINAL SUMMARY REPORT HIGH POWER DENSITY DEVELOPMENT PROJECT BIG ROCK POINT REACTOR DEVELOPMENTAL FUEL TESTING
}

V. E. HAZEL R. N. DUNCAN

U. S. ATOMIC ENERGY COMMISSION

CONTRACT AT(04-3)-361 


\section{DISCLAIMER}

This report was prepared as an account of work sponsored by an agency of the United States Government. Neither the United States Government nor any agency Thereof, nor any of their employees, makes any warranty, express or implied, or assumes any legal liability or responsibility for the accuracy, completeness, or usefulness of any information, apparatus, product, or process disclosed, or represents that its use would not infringe privately owned rights. Reference herein to any specific commercial product, process, or service by trade name, trademark, manufacturer, or otherwise does not necessarily constitute or imply its endorsement, recommendation, or favoring by the United States Government or any agency thereof. The views and opinions of authors expressed herein do not necessarily state or reflect those of the United States Government or any agency thereof. 


\section{DISCLAIMER}

Portions of this document may be illegible in electronic image products. Images are produced from the best available original document. 
AEC Research and

Development Report

March 1972

FINAL SUMMARY REPORT

\section{HIGH POWER DENSITY DEVELOPMENT PROJECT: \\ BIG ROOCK POINT REACTOR DEVELOPMENTAL FUEL TESTING}

V. E. Hazel

R. N. Duncan

Approved

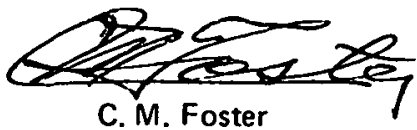

Project Engineer
Approved:

\section{. Approved}

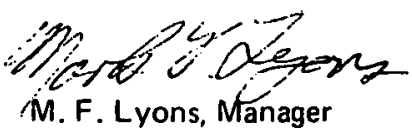

iM. F. Lyons, Manager

Fuel Development
Prepared for the

U.S. Atomic Energy Commission

Contract AT(04-3)-361
This report was prepared as an account of work Thonsored by the United States Government Neither the United States nor the United States Atomic Energy Comir Comission, nor any of their employes, nor any of their contractors, subcontractors, or their employees, makes any warranty, express or implied, or assumes any egal liability or responsibility for the accuracy, completeness or usefulness of any information, apparatus, product or process disclosed, or represents that its use would not infringe privately owned rights.

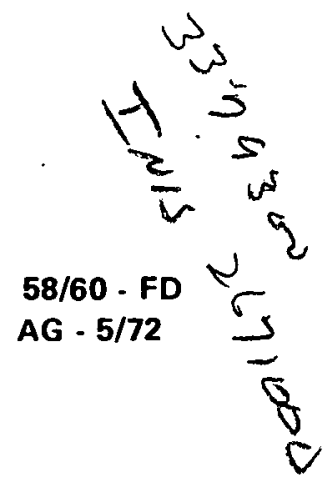

NUOLCAR FUEL DETANTMEPST — OENEAAL ELECTMIC COMPANY SAN JOSE, CALIFORNIA 95114

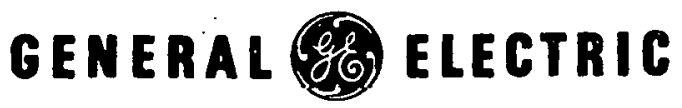




\section{NOTICE}

This report was prepared as an account of work sponsored by the United States Government. Neither the United States nor the United States Atomic Energy Commission, nor any of their employees, nor any of their contractors, subcontractors, or their employees, makes any warranty, express or implied, or assumes any legal liability or responsibility for the accuracy, completeness or usefulness of any information, apparatus, product or process disclosed, or represents that its use would not infringe privately owned rights.

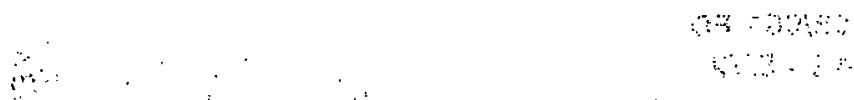


TABLE OF CONTENTS

1. INTRODUCTION AND SUMMARY OF RESULTS

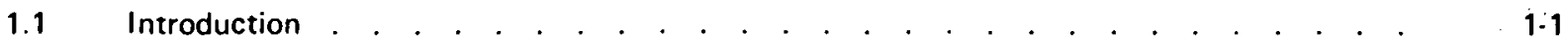

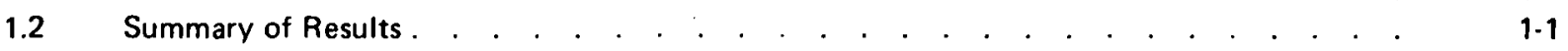

2. DEVELOPMENTAL FUEL DESIGN AND FABRICATION $\quad . \quad$. . . . . . . . . . . . . . . . . . $\quad 2.1$

2.1 Design and Performance Criteria for Fuel Performance Tests in BRP . . . . . . . . . . . . $\quad 2-1$

2.2 Assembly Structure . . . . . . . . . . . . . . . . . . . . . . . . . . 2.1

2.3 Fuel Rod Design and Fabrication . . . . . . . . . . . . . . . . . . . . . . . . . . . .

3. FUEL PERFORMANCE . . . . . . . . . . . . . . . . . . . . . . . . . . . 3-1

$3.1 \quad$ Irradiation History $\quad . \quad$.

3.2 Interim Examinations . . . . . . . . . . . . . . . . . . . . . . . . . . . . . . . . . . . .

3.2.1 Visual Inspections . . . . . . . . . . . . . . . . . . . . . . . . . . . . 31

3.2.2 Dimensional Profiles . . . . . . . . . . . . . . . . . . . . . . . . 3-1

3.2.3 Post-Irradiation Examination of Unfailed Rods . . . . . . . . . . . . . . . . . 3-11

4. FAILURE DESCRIPTIONS AND ANALYSIS . . . . . . . . . . . . . . . . . . . . . . . 4-1

4.1 Type 304-L Clad Fuel Rods . . . . . . . . . . . . . . . . . . . . . . . . . . . . . . . . . . . . . . . 4.1

4.1.1. Visual Inspection of Developmental Assemblies Containing Stainless
Steel Clad Fuel Rods During the April 1966 Shutdown . . . . . . . . . . . . . . . . . $4-1$

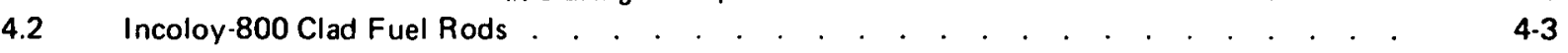

4.2.1 Inspection of Incoloy-800 Clad Fuel Rods in Developmental Assemblies
During Reactor Shutdowns 1966 and May $1967 \quad$. . . . . . . . . . . . . . . 4-3

4.2.2 Visual Examination Results for Assemblies D-10, D-11, D-12, D-13,

D-14, and D-15 . . . . . . . . . . . . . . . . . . . . . . . 4 46

4.2.3 Post-Irradiation Examination of Failed Incoloy-800 Fuel Rod Cladding . . . . . $\quad 4-6$

4.2.4 Intergranular Corrosion Susceptibility Tests on Incoloy-800 . . . . . . . . . 4

$4.3 \quad$ Zircaloy-2 Clad-UO $\mathrm{U}_{2}$ Powder Fuel Rods . . . . . . . . . . . . . . . . . . . . . . . . . . . . . $\quad 4-13$

4.3.1 Nondestructive Examination . . . . . . . . . . . . . . . . . . . 4 4-15

4.3.2 Metallography. . . . . . . . . . . . . . . . . . . . . . . . 48

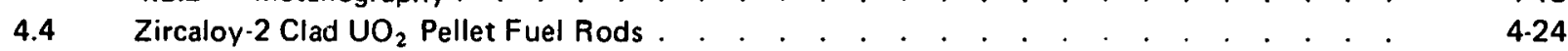

REFERENCES . . . . . . . . . . . . . . . . . . . . . . . . . . . . . . . . . . . . . . .

\section{APPENDIX}

UNDERWATER PROFILOMETER $\quad . \quad$. . . . . . . . . . . . . . . . . . . . . . . . . . . . .

DISTRIBUTION . . . . . . . . . . . . . . . . . . . . . . . . . . . . . . . . . . . . 


\section{LIST OF ILLUSTRATIONS}

Profile.Traces, $\Delta D$ versus $L$, of Irradiated Fuel Rod from Assembly D-2

Showing Ridging of Cladding .

Profile Trace, $\Delta D$ versus $L$, of Freestanding Irradiated Fuel Rod from

Assembly D-2 Showing Local Ovality of Cladding . 


\section{LIST OF ILLUSTRATIONS (Continued)}

Corrosion Behavior of Various Incoloy-800 Samples in $\mathrm{HNO}_{3}-\mathrm{Cr}^{+6}$ Solution . . . . . . . . 4-14

End Region of Zircaloy-2 Clad Rod CR-163, Showing that the Lower End Plug is Missing . . . 4-16

Part of Lower End of Rod CR-163 Wedged at the Lower Spacer, Band of Assembly D-19 . . . $\quad 4-16$

An Intact Lower End Plug of a Zircaloy-2 Clad Powder Fuel Rod.

Circumferential Crack at Top of Zircaloy-2 Clad Rod CR-163 after Initial Cutting

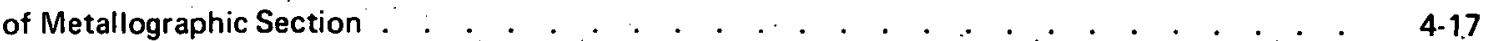

Comparison Neutron Radiographs of Zircaloy-2 Clad Fuel Rods CR-163 and

CR-125, Top Énd

First Metallographic Plane of CR-163, Upper Failure 


\section{INTRODUCTION AND SUMMARY OF RESULTS}

\subsection{INTRODUCTION}

The uriginal objectives of the High Power Density Development Fuel Program were to (a) design and fabricate fuel assemblies using features, materials, and processes having a potential for reduced fabrication costs, and (b) determine performance capabilities of these fuel assemblies when irradiated at a core average power density of 45 to 60 $\mathrm{kW} /$ liter up to average rod exposures of $15,000 \mathrm{MWd} / \mathrm{TU}$ in a boiling water reactor (BWR).

Type-304 stainless steel was originally chosen as the reference cladding material because it was readily available, could be adapted to a variety of fabrication methods, and appeared to be entirely suitable for a BWR environment, based on information available at the start of the program. In addition, the early, relatively high cost of Zircaloy tubing made thin Type-304 stainless steel cladding economically competitive with Zircaloy. When Type-304 stainless steel cladding began to fail, a third objective was added: (c) investigate the performance of alternate cladding materials, keeping the low-cost emphasis if possible.

\subsection{SUMMARY OF RESULTS}

- All of the developmental assemblies were irradiated in an 84-bundle core operated at a core average power of $45 \mathrm{~kW}$ /liter. Four of the developmental assemblies, D-1, -2, -3, and -8, operated at a life-average power density of $45 \mathrm{~kW} /$ liter up to their design life of $15,000 \mathrm{MWd} / \mathrm{TU}$.

Assemblies D-1, -2, and -3, with drawn Zircaloy-2 cladding, $\mathrm{UO}_{2}$ pellet fuel, and the spacer and structure configuration typical of the BRP development assemblies, may have encountered a life limit in the range of 15,000 to $17,000 \mathrm{MWd} / \mathrm{TU}$ exposure. One of the three assemblies, D-2, was found to be leaking when sipped at $16,900 \mathrm{MWd} / \mathrm{TU}$ exposure. However, the fuel rod(s) responsible for the leak signal could not be located during the limited visual inspection performed. Both D-1 and D-3 were unfailed at discharge exposures of 16,900 and $19,000 \mathrm{MWd} / \mathrm{TU}$, respectively.

Even if the apparent life limit suggested by the performance of assembly D-2 were real, the successful operation of the Zircaloy-clad rods to this exposure makes austenitic cladding distinctly uneconomical by comparison. Thus, the successful operation of the Inconel-600 clad bundle D-8 to somewhat higher exposures is irrelevant to achieving the lowest fuel cycle cost.

- The failure of Type-304L stainless steel clad fuel is consistent with results obtained in the VBWR irradiations.

- Failures that could not be attributed directly to single variables under test prevented performance comparison of many of the various parameters. For example, both rocked and drawn, Zircaloy-2 clad, $\mathrm{UO}_{2}$ powder fuel failed early in life, apparently from hydrogenous impurities. Thus, the rocked and drawn tubing performance could not be compared at high exposures.

- Early failure of $\mathrm{UO}_{2}$ powder fuel rods from hydrogenous impurities precludes performance comparisons with $\mathrm{UO}_{2}$ pellet fuel. However, the apparent influence of impurities in failure of Zircaloy-2 clad powder fuel (D-18, $-19,-20)$ indicates that more stringent or added process steps are needed in tabrication of $\mathrm{UO}_{2}$ powder fuel rods. The additional required quality control steps may offset the economic advantages for the powder compaction process in high volume production (unless similar measures also prove necessary for pellet fuel). 


\section{DEVELOPMENTAL FUEL DESIGN AND FABRICATION}

\subsection{DESIGN AND PERFORMANCE CRITERIA FOR FUEL PERFORMANCE TESTS IN BRP ${ }^{1}$}

a. The development assemblies were to be compatible with physics and thermal-hydraulic parameters of the standard fuel in the core.

b. The fuel assembly structure was to be low in cost, and accommodate length variations among fuel rods.

c. Individual fuel rods were to be easily insertable and removable for ease of inspection and replacement.

d. End plug geometry was to be simple for low cost.

e. Fuel rods were to use cladding purchased to commercial tolerances and specifications.

f. The fuel was to be uranium dioxide, clad with Type-304 stainless steel. (Thiscriterion was modified for use of alternate cladding alloys.)

g. The assemblies were to operate at 45 to $60 \mathrm{~kW} /$ liter average power density to 10,000 to $15,000 \mathrm{MWd} / \mathrm{TU}$ average exposure.

\subsection{ASSEMBLY STRUCTURE}

The structure is the metal subassembly that contains and positions the fuel rods. It consists of a base, corner angles, spacers, weldment, and handle, as shown in Figures 1 and 2. Parts are welded construction: Type-304 stainless steel for the first eight assemblies, and Incoloy-800 for the remaining assemblies. The fuel rod ends are not attached to the structure so that simple end plugs could be used. The fuel rods rest on the base and are positioned laterally by intermediate spacers.

The PE, PO, and D-1 through -15 assemblies used seven intermediate spacers at an axial spacing of 11-1/4 inches. Four of the spacers were double-layer wire spacers alternated with three single-layer wire spacers, as shown in Figure 2. The D-16 through - 20 assemblies used six double-layer wire spacers at 13-1/2-inch spacing. In addition, the spacers for D-18, -19, and -20 incorporated an Inconel-X strip between alternate rows of fuel rods, with cantilever prongs which acted as springs to bear against the fuel rods to reduce vibration and fretting wear.

The handle prevented upward movement of the fuel rods in excess of the initial vertical clearance allowance of up to $3 / 4$ inch. Thus, a $3 / 4$-inch length variation in fuel rods could be accommodated. The handle was held in place by the locking pin passing through the weldment. The weldment, in turn, was attached to the corner angles which support and position the spacers and base. Removing the locking pin permitted removal of the handle and allowed access to remove any or all of the fuel rods.

\subsection{FUEL ROD DESIGN AND FABRICATION}

Three groups of fuel assemblies were built under the Development Fuel Program. Group I and Group II were fabricated and initially irradiated under Phase I and Phase II, respectively, of the HPD Program. ${ }^{2},{ }^{3} \mathrm{Group}$ III was then fabricated to provide assemblies with additional variables. The design variables for the development assemblies are summarized in Section 3, Table 1. Many variables were included so that a broad comparison of fabrication parameters and their effects on fuel performance could be made.

Fuel forms evaluated were sintered $\mathrm{UO}_{2}$ pellets, vibratory compacted $\mathrm{UO}_{2}$ powder (both arc-fused and Dynapak), and swaged $\mathrm{UO}_{2}$ powder (arc fused),

The sintered pellet process consists of: (a) hydrostatically pressing pellets made from ceramic-grade uranium-dioxide powder to 50 to $60 \%$ of theoretical density and then sintering in 1600 to $1700^{\circ} \mathrm{C}$ hydrogen until the density reaches 94 to $97 \%$ of theoretical; (b) centerless grinding the pellets to size; (c) drying the pellets at $120^{\circ} \mathrm{C}$; and (d) loadıng the pellets in tubes, backfilling with helium, and sealing by welding end plugs in the tubes. ${ }^{4}, 5$ This summarizes the standard pellet fuel rod fabrication process. The swaged pellet process adds a swaging step, applied to the finished rod, to eliminate the pellet-cladding gap and thereby reduce wrinkling susceptibility. ${ }^{6}$ 

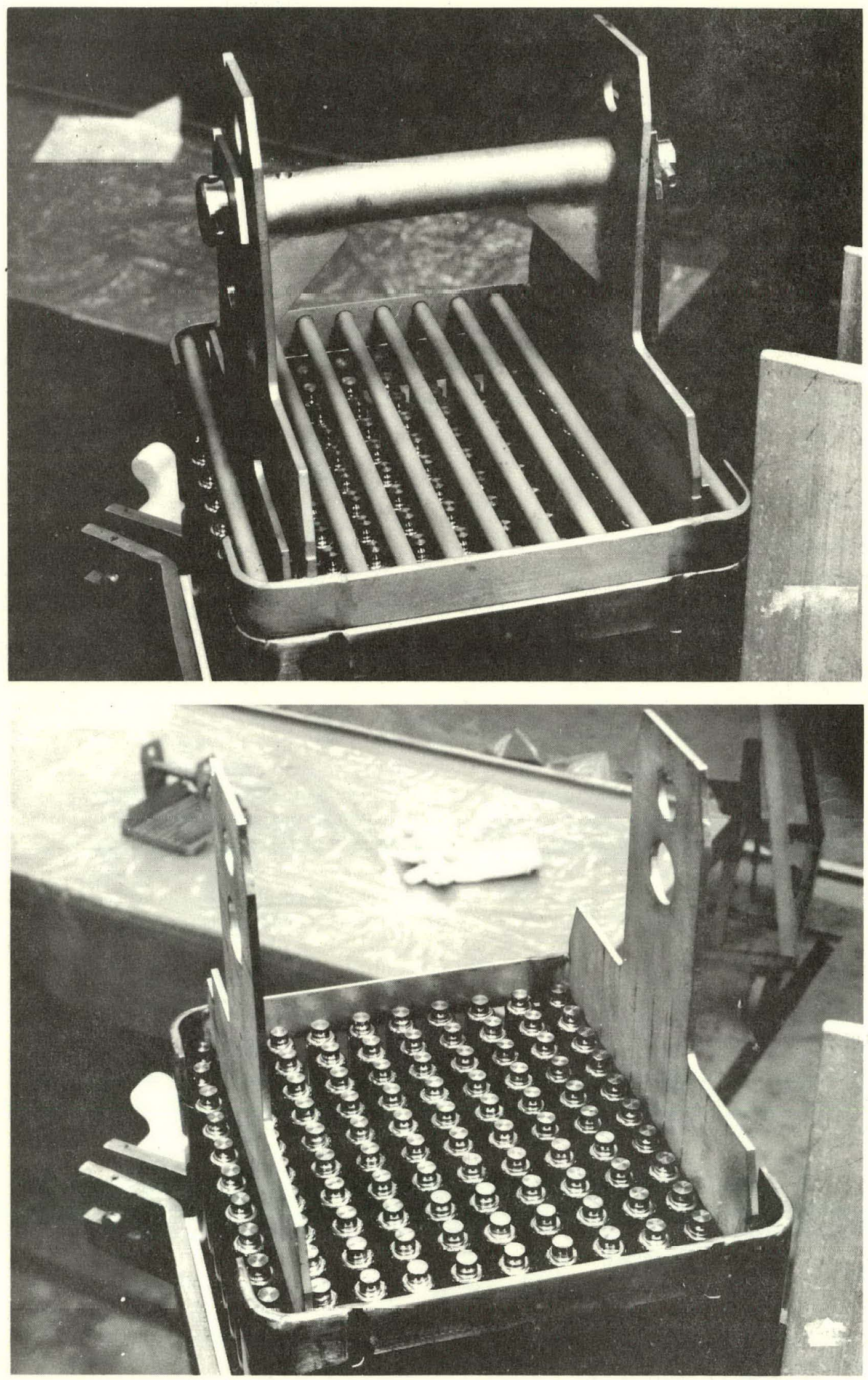

Figure 1. Phase // Fuel Element, Removable Handle and Upper Grid 


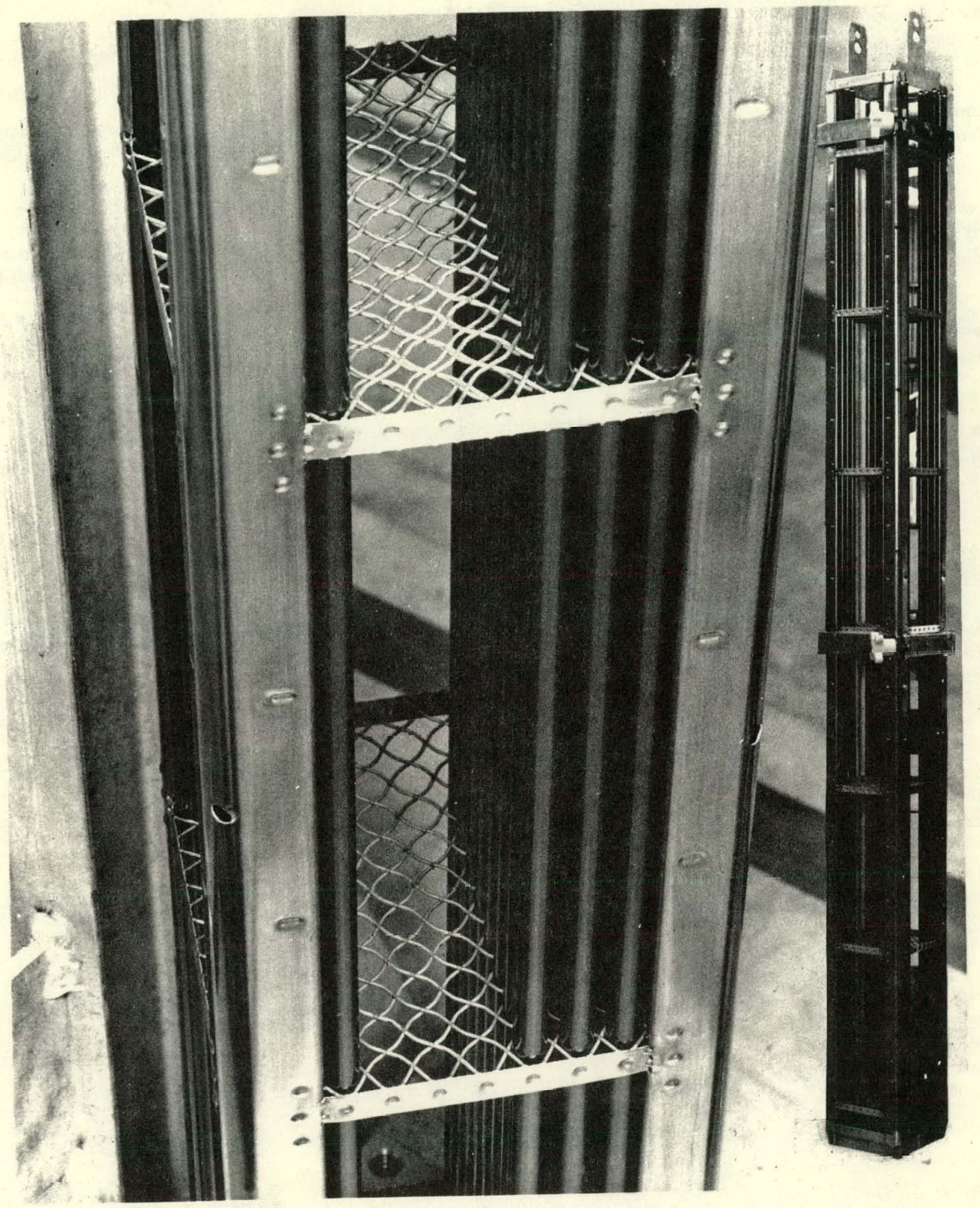

Figure 2. Spacer and Structure Details, Partially Assembled Element 
The vibratory compaction process consists of loading high-density ( 98 to $99+\%$ of theoretical) $\mathrm{UO}_{2}$ particles ${ }^{*}$ of carefully selected size distributions ${ }^{7}$ into the cladding tube, vibrating to 82 to $88 \%$ of theoretical density, and then sealing the tube.

The swaged powder process consists of loading and sealing the tubes as above for the straight vibratory compaction process, and then swaging the loaded tubes to smaller diameter and higher $(>90 \%$ of theoretical) fuel density. ${ }^{6}, 8$

Cladding processing conditions were mill-annealed (austenitic alloys), mill-annealed and lightly ( 1 to $2 \%$ cold work) swaged in the case of swaged pellet fuel (austenitic alloys), mill-annealed and heavily ( 10 to $30 \%$ cold work) swaged in the case of swaged powder fuel (austenitic alloys), and drawn or rocked to 15 to $70 \%$ cold work and stress relieved (Zircaloy-2).

Each developmental assembly contained 121 fuel rods in an $11 \times 11$ square array. All, except the 12 corner rods, were 0.425 inch o.d. at a 0.575 -inch pitch. The corner rods were smaller, 0.320 inch o.d., to reduce fuel rod power and allow sufficient clearance for proper cooling. Active fuel length was 68 to 70 inches.

A plenum was provided in each fuel rod to keep fission gas induced internal pressure low. This plenum was above the fuel column for pellet fuel and distributed through the 8 to $15 \%$ voids in the fuel column for compacted powder fuel. An Inconel-X 750 spring was used in the pellet-rod plenum to hold the fuel column in place and, in the case of thin-clad rods, to support the cladding against the external coolant pressure.

The Group I fuel assemblies were the next development step after the BRP first-core fuel which consisted of 0.388 o.d. $\times 0.019$ wall Type-304 stainless steel clad $\cup_{2}$ pellet fuel. The Group I assemblies were designed to utilize the most promising (both for technical and low-cost considerations) fabrication processes evaluated earlier in the Vallecitos Boiling Water Reactor irradiations. ${ }^{9}$ These were thin-wall clad, sintered $\mathrm{UO}_{2}$ pellet fuel and thin-wall clad, swaged $\mathrm{UO}_{2}$ powder fuel. The thin-wall cladding absorbs fewer neutrons than thicker cladding and so reduces initial enrichment costs and/or extends fuel reactivity for higher burnup capability. The swaged powder fuel was believed to have reduced cost potential by eliminating several steps required in pellet fuel fabrication while maintaining the equivalent smeared density and therefore quantity of $\mathrm{UO}_{2}$ in the fuel rod.

Eight Group I fuel assemblies, designated PE-1 to PE-4 and PO-1 to PO-4, were made. Cladding was Type-304 stainless steel for PE-1 and PE-2 fuel rods, and Type-304L stainless steel for PE-3, PE-4, and all PO rods. Type-304 stainless steel was replaced by Type-304L stainless steel to reduce susceptibility to grain boundary sensitization and subsequent localized corrosion.

Cladding thickness was 0.010 inch for all PE and PO assemblies. Collapsible cup-type end plugs ${ }^{10}$ were used for all developmental swaged powder fuel rods, thus eliminating the extra steps of attaching and removing temporary end plugs.

The primary purpose of the 15 Group II fuel assemblies was to determine the performance of fuel clad with alternate materials. The alternate materials were Zircaloy-2, Incoloy-800, and Inconel-600. Zircaloy-2 was selected on the basis of successful operation on other programs and its low neutron absorption cross section. ${ }^{\dagger}$ Incoloy-800 and Inconel-600 were selected on the basis of improved general corrosion resistance over Type-304 stainless steel, and the demonstrated superior Incoloy-800 and Inconel-600 performance with respect to intergranular attack compared with Type-304 stainless steel in the superheat program. ${ }^{11}, 12$

* The particles can be either arc-fused or "Dynapak" $\mathrm{UO}_{2}$. The arc-fused material is generally higher in initial density but has lower useful yield than the Dynapak.

† Another factor favoring Zircaloy was its decline in price with time. Thus, a cladding material of high neutron absorption would now have to be much thinner to compete in fuel cycle costs with Zircaloy clad fuel than it would have had to be at the start of the High Power Density Program. 
The first nine Group II assemblies consisted of thick-clad (freestanding), large fuel-to-cladding gap, pellet-containing fuel rods to give a high probability of successful operations. Three assemblies, designated D-1 to D-3, were clad with 0.030 -inch wall, drawn Zircaloy-2, and had a nominal fuel-to-cladding diametral gap of 7 mils. The next three, D-4 to D-6, were clad with 0.019 -inch wall, weld-drawn Incoloy-800 with a nominal fuel-to-cladding gap of 6 mils: Assemblies D-7 to D-9 were identical to D-4 through D-6 except the cladding material was Inconel-600 rather than Incoloy-800.

The remaining six Group II assemblies consisted of 0.011 -inch wall, Incoloy-800 clad, swaged powder fuel rods, essentially duplicating the Group I PO assemblies except for cladding material.

Part of the Phase $1 \mathrm{l}$ testing involved a core power flattening experiment using $\mathrm{B}_{4} \mathrm{C}$ or gadolinia-urania poison reds to replace four fuel rods in several developmental assemblies. ${ }^{3}, 13,14$ The five Group III developmental assemblies were an extension of the Group II Program, incorporating thinner (0.011-inch wall) Incoloy-800 clad pellet fuel in two assemblies (D-16 and D-17), and 0.030-inch wall, Zircaloy-2 clad, vibratory-compacted powder fuel in three assemblies (D-18 to D-20).

One other significant feature of the two Incoloy-800 clad assemblies, D-16 and D-17, was the use of seamless tubing. Prior experience with welded Incoloy-800 tubing indicated a visibly nonhomogenous zone at the seam weld, requiring special heat treatments to homogenize the weld. ${ }^{15}$ Using seamless tubing eliminated any question of reduced integrity related to the seam weld zone.

The D-18 to D-20 fuel rods contained a 0.012 -inch-thick, cupped, close-fitting, Zircaloy-2 disc at the top of the fuel column to keep the $\mathrm{UO}_{2}$ away from the end plug during welding. The powder fuel rods fabricated earlier (i.e., before D-18, D-19 and D-20) used a stainless steel wool plug to separate the fuel from the end plug. 


\section{FUEL PERFORMANCE}

\subsection{IRRADIATION HISTORY}

Irradiation of the PE and PO assemblies began during the summer of 1963 , bundles D-1 to D-15 began irradiation during the summer of 1964 , and the D-16 to D-20 bundle irradiations began during the summer of 1965 . Table 1 lists the exposure, heat flux, and irradiation time for all the developmental assemblies. Coolant pressures during initial reactor testing ranged from 1000 to $1500 \mathrm{psi}$. However, the subsequent irradiation has been at a pressure of 1300 to 1350 psi $\left(\sim 580^{\circ} \mathrm{F}\right.$ saturation temperature $)$. Heat fluxes up to $430,000 \mathrm{Btu} / \mathrm{h}-\mathrm{ft}^{2}(14 \mathrm{~kW} / \mathrm{ft})$ were attained by some developmental assemblies during 1964 while testing 44-bundle, $60 \mathrm{~kW} / \mathrm{liter}$ average power density cores. $^{14}$ Subsequently, the maximum heat flux of the developmental assemblies was in the range 300,000 to $350,000 \mathrm{Btu} / \mathrm{h}-\mathrm{ft}^{2}$ (9 to $11 \mathrm{~kW} / \mathrm{ft}$ ).

\subsection{INTERIM EXAMINATIONS}

The developmental assemblies were periodically given visual and dimensional examinations. These examinations were to determine any structure deterioration that might interfere with continued assembly irradiation, such as warped structure, damaged spacers, or bowed fuel rods, and to determine appearance and dimensional stability of the fuel rods. The interim examination results are summarized in Table 2.

\subsubsection{Visual Inspections}

Visual examinations were performed using an underwater periscope, closed-circuit television, and/or binoculars. No deformation or rod bowing was observed during any of the interim examinations. Surface deposit buildup on the rods prevented observation of cladding oxide changes with time. The deposits built up in a pattern which was influenced by power distribution, fuel rod position, and spacer loaction. A long, narrow, spear-point-shaped deposit pattern, called "streaming," developed just above the central spacer bands of all the developmental assemblies, regardless of type. In addition, a bamboo-like pattern of circumferential striations was observed. This "bambooing" was prominent on pellet fuel rods, and usually the striations corresponded to pellet interfaces. As surface deposit buildup occurred, the deposits began spalling off the austenitic cladding materials. Spalling from the Zircaloy-2 clad rods was much less and occurred later in life. Small black areas were observed at several of the interim examinations which were probably local spalling from the Zircaloy-2 (see, for example, Figure 3).

Cladding failures were seen in assemblies PE-3, D-10, D-11, D-12, and D:13. The failures were generally longitudinal splits in the cladding, although PE-3 had sume circumferential cracks also. (See Section 4 for additional details.)

\subsubsection{Dimensional Profiles}

Dimensional prnfiles were manle ussing an underwater profilometer capable of recording fuel rod diameter versus length (axial), angle (circumferential), or combined coordinates (helical traces) Additional profilometer performance information is given in the Appendix.

The first profilometer measurements included fuel rods with $1500 \mathrm{MWd} / \mathrm{TU}$ exposure (2 to 3 months). At this relatively low exposure, periodic diameter increases at pellet length axial intervals (circumferential ridges) were observed, as well as up to 6 mils local ovality (see Figures 4 and 5). The diameter increases were attributed to cladding plastic strain as a result of pellet-to-cladding interactions. Later measurements indicated additional diameter increases and ovality which were attributed to nonuniform deposit buildup on the cladding surface (see Figures 6 and 7). Additional measurements indicated a process of deposit buildup and spalling for the austenitic cladding materials in agreement with the visual observations. An extreme example of deposit spalling is shown in Figure 8, where the upper and lower limits of previous diameter measurements were compared with a later trace of a fuel rod from assembly D-5. The Zircaloy-2 clad rods did not show the marked diameter decreases observed with the spalling austenitic cladding, but some measurements after $7500 \mathrm{MWd} / \mathrm{TU}$ indicated either slight deposit spalling or creep collapse of the Zircaloy-2. Compare Figures 5, 6, and 7 . There are regions on the later profile traces which indicate smaller local diameters than the earlier traces. 


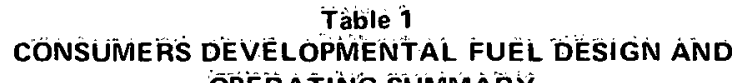

UO $O_{2}$ Füe

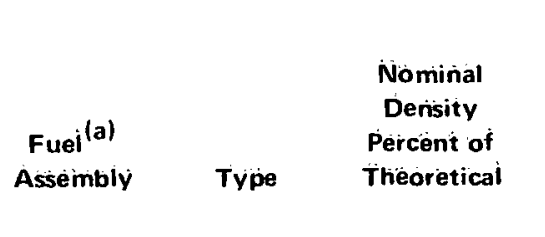

Cladding

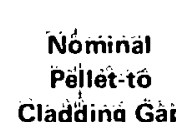

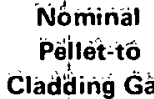

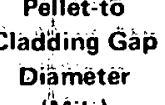

Diânimetềr
('Mils).

$$
\text { PE-T }
$$

PE-2

PE-3 Pellet

PE-4 Pellet

PO

PO-

$\overline{\mathrm{P}} \mathrm{O}-\dot{3} \quad$ Powder

PO-4 Powder

Group II Peilet

D-2 Pellet

D:3 Pellet

D-4 Pellet

D.5 Pellet

D-6 Pellet
Type-304

-Type-304

Stainilèss Stèel

Type-304L
Stäiñless Steèl

Type-30iouL
Staintess Śteel

Stainless Stéel

Type-304L

Śtairiless Steèt

Typè-304L
Stainless Steel

ivpe-jüL L

Stainlless Steé

Type-30̈4L
Stainless Stee

Zircaioy-2
(Drawn)

Zircaloy-2
(Driwnri)

Zircalov-2
(Drawn)

(Drawn)

Incolov-800
(Weld-drawn)

incoloy 800

Weld-drawinin

Incoloy-80́o
(Weld-drawn)
0 to 2

0 to $2 \quad 0.010$

0 to $2 \quad 0.010$

0.010

0.010

0.010

0.010

0.030

0.030

0.019

o.019

0.019
Fabricátion Process

Total
Accumulated Exposiuré

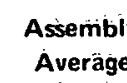

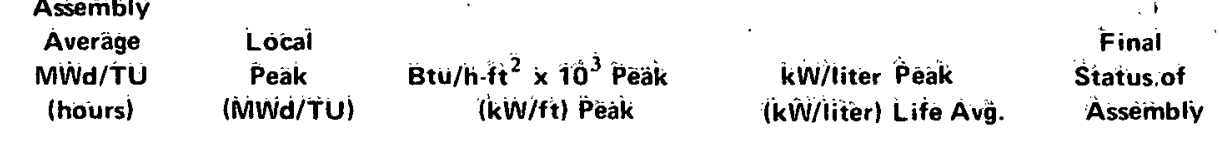

Condition. $\mathrm{VO}_{2}$ Fuel

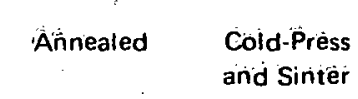

$\begin{array}{lc}\text { Swaje Over } & 3.425 \\ \text { Unigroüund } & (5.125) \\ \text { Pellețs } & \end{array}$

$\begin{array}{lr}\text { Swagè Over } & 3,340 \\ \text { Gröund Pêllèt } & (5,125)\end{array}$

11000

Ground Peillets $(10,500)$

$\begin{array}{lr}\text { Swagè Över } & 5,640 \\ \text { Groùnd Pëliets } & 15,200\end{array}$

Swäğe $\quad 6.570$

T2.300)

$\begin{array}{lc}\text { Suwage } & 7,680 \\ \text { Compaction } & (10,500)\end{array}$

$\begin{array}{ll}\text { Cowogi } & 6,600 \\ \text { Compaction } & (1,800)\end{array}$

Swäge $\quad 7.035$

7.035
$13.600)$

Stàndàrd $\quad \begin{gathered}6,850 \\ (21,200)\end{gathered} \quad 222,400$

Coid-Wörked Cold:Press
(15 to $25 \%$ a and Sinter

Coldt:Worked Cold-Press

Cold-Worked Colid:Prèss

(15 to 25\%) and Sinter

$\begin{array}{cc}\text { Anneäled } & \begin{array}{c}\text { Cold-Press } \\ \text { añid Sinter }\end{array} \\ & \end{array}$

Añnealed Cold:Press

Cold: Pिress
and Sinter

Annealed Cold-prests
and Sinter
Standarard $\quad 16,470 \quad 21,900$

16,470
$(19,000)$

$\begin{array}{r}19.100 \\ 127,100)\end{array} \quad 25,400$

13,900

Ständard

$(12,100)$

Stándar

12,860
$(21,200$

12,600
Stañdä̀rd $\quad(21,200)$

17,600
136.
146.11

136
145.2) Out of Core

142
$(4990)$

$142 . \quad$ Out of cơrè

129 Out of corè

149
$149.3)$

128 niti if r.nire

123 Out ồ core

(34.8)

(42.3) ${ }^{13 \hat{\bar{\sigma}}} 45 . .^{(\hat{b})}$ Out or Cure

(i1.4)

(11.6)

$(45.8)^{138} 45.7^{(b)} \quad \begin{gathered}\text { Out of Core } \\ \text { (Suspect) }\end{gathered}$

$137.213145 . .^{(b)}$ Out of Córe

133
$148.5)$

$16 \overline{7}$
$(3 \overline{6} .1)$

167
135.31 
$\mathrm{UO}_{2}$ Fuel

\begin{tabular}{|c|c|c|c|c|c|c|c|}
\hline \multirow[b]{2}{*}{$\begin{array}{l}\text { Fuel (a) } \\
\text { Assombly }\end{array}$} & \multicolumn{2}{|c|}{$\mathrm{UO}_{2}$ Fuel } & \multicolumn{3}{|c|}{ Cladding } & & Fabri \\
\hline & Type & $\begin{array}{l}\text { Nominal } \\
\text { Density } \\
\text { Percent of } \\
\text { Theoretical }\end{array}$ & $\begin{array}{l}\text { Tubing } \\
\text { Material }\end{array}$ & $\begin{array}{l}\text { Nominal } \\
\text { Pellet-to } \\
\text { Cladding Gap } \\
\text { Diameter } \\
\text { (Mils) }\end{array}$ & $\begin{array}{c}\text { Nominal } \\
\text { Wall } \\
\text { (inch) }\end{array}$ & Condition & $\mathrm{UO}_{2}$ Fuel \\
\hline 0.7 & Pellet & 94 & $\begin{array}{l}\text { Inconel-600 } \\
\text { (Weld-drawn) }\end{array}$ & 6 & 0.019 & Annealed & $\begin{array}{l}\text { Cold-Press } \\
\text { and Sinter }\end{array}$ \\
\hline D-8 & Pellet & 94 & $\begin{array}{l}\text { Inconel-600 } \\
\text { (Weld-drawn) }\end{array}$ & 6 & 0.019 & Annealed & $\begin{array}{l}\text { Cold-Press } \\
\text { and Sinter }\end{array}$ \\
\hline $0-9$ & Peller & 94 & $\begin{array}{l}\text { Inconel-600 } \\
\text { (Weld-drawn) }\end{array}$ & 6 & 0.019 & Annealed & $\begin{array}{l}\text { Cold-Press } \\
\text { and Sinter }\end{array}$ \\
\hline D. 10 & Powder & 91 & $\begin{array}{l}\text { Incoloy. } 800 \\
\text { (Weld-drawn) }\end{array}$ & 0 & 0.011 & Cold-Worked & Arc Fuse \\
\hline$D-11$ & Powder & 91 & $\begin{array}{l}\text { Incolov-800 } \\
\text { (Weld-drawn) }\end{array}$ & 0 & 0.011 & Cold-Worked & Arc Fuse \\
\hline D.12 & Powder & 91 & $\begin{array}{l}\text { Incolov-800 } \\
\text { (Weld-drawn) }\end{array}$ & 0 & 0.011 & Cold-Worked & Arc Fuse \\
\hline$D-13$ & Powder & 91 & $\begin{array}{l}\text { Incoloy-800 } \\
\text { (Weld-drawn) }\end{array}$ & 0 & 0.011 & Cold-Worked & Arc Fuse \\
\hline D.14 & Powder & 91 & $\begin{array}{l}\text { Incolov-800 } \\
\text { (Weld-drawn) }\end{array}$ & 0 & 0.011 & Cold-Worked & Arc Fuse \\
\hline 0.15 & Powder & 91 & $\begin{array}{l}\text { Incoloy-800 } \\
\text { (Weld-drawn) }\end{array}$ & 0 & 0.011 & Cold-Worked & Arc Fuse \\
\hline $\begin{array}{l}\text { Group III } \\
\text { D-16 }\end{array}$ & Pellet & 94 & $\begin{array}{l}\text { Incoloy-800 } \\
\text { (Seamless) }\end{array}$ & 0101 & 0.011 & Annealed & $\begin{array}{l}\text { Cold-Press } \\
\text { and Sinter }\end{array}$ \\
\hline D-17 & Pellet & 94 & $\begin{array}{l}\text { Incolov-800 } \\
\text { (Seamless) }\end{array}$ & 0 to 1 & 0.011 & Annealed & $\begin{array}{l}\text { Cold-Press } \\
\text { and Sinter }\end{array}$ \\
\hline D-18 & Powder & $83-85$ & $\begin{array}{l}\text { Zircaloy-2 } \\
\text { (Rocked) }\end{array}$ & 0 & 0.030 & $\begin{array}{l}\text { Cold-Worked } \\
\text { (50 to } 70 \%)\end{array}$ & Dynapak \\
\hline D-19 & Powder. & 83-85 & $\begin{array}{l}\text { Zircaloy-2 } \\
\text { (Rocked) }\end{array}$ & 0 & 0.030 & $\begin{array}{l}\text { Cold-Worked } \\
\text { (50 to } 70 \% \text { ) }\end{array}$ & Dynapak \\
\hline D-20 & Powder & 83-85 & $\begin{array}{l}\text { Zircalovy-2 } \\
\text { (uranw) }\end{array}$ & 0 & 0.030 & $\begin{array}{l}\text { Cold- Worked } \\
1155025 \%)\end{array}$ & Dynapak \\
\hline
\end{tabular}

Table 1 (Continued)

abrication Process

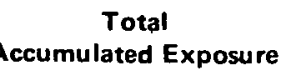

Fuel Rod Ratings Experienced

\begin{tabular}{|c|c|c|c|c|c|}
\hline Fuel Rod & $\begin{array}{l}\text { Assembly } \\
\text { Average } \\
\text { MWd/Tu } \\
\text { (hours) }\end{array}$ & $\begin{array}{l}\text { Lecal } \\
\text { Peak } \\
(M W d / T U)\end{array}$ & 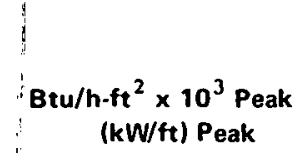 & $\begin{array}{c}\mathrm{kWW/iter} \text { Peak } \\
(\mathrm{kW} / \mathrm{iter}) \text { Life. Ave. }\end{array}$ & $\begin{array}{l}\text { Final } \\
\text { Status of } \\
\text { Assembly }\end{array}$ \\
\hline Standard & $\begin{array}{l}13,040 \\
(20,000)\end{array}$ & $18,250$. & $\begin{array}{l}327 \\
(10.7)\end{array}$ & $\begin{array}{l}127 \\
(38.9)\end{array}$ & $\begin{array}{l}\text { Out of Core } \\
\text { (Suspect) }\end{array}$ \\
\hline Standard & $\begin{array}{c}15,760 \\
(21,200)\end{array}$ & 21,000 & $\begin{array}{l}430 \\
(14.0)\end{array}$ & $(44.5)^{167} 48.2^{(b)}$ & Out of Core \\
\hline Standard & $\begin{array}{l}15,700 \\
(27,100)\end{array}$ & 20.900 & $\begin{array}{l}430 \\
(14.0)\end{array}$ & $(34.7)^{167}{ }_{36.5^{(b)}}$ & In-Core \\
\hline $\begin{array}{l}\text { Swage } \\
\text { Compaction }\end{array}$ & $\begin{array}{c}6,650 \\
(9,425)\end{array}$ & 9.750 & $\begin{array}{l}324 \\
(10.6)\end{array}$ & $\begin{array}{c}126 \\
(46.5)\end{array}$ & Failed \\
\hline $\begin{array}{l}\text { Swage } \\
\text { Compaction }\end{array}$ & $\begin{array}{l}6,750 \\
(9,425)\end{array}$ & 9,900 & $\begin{array}{c}389 \\
(12.7)\end{array}$ & $\begin{array}{c}151 \\
(47.2)\end{array}$ & Failed \\
\hline $\begin{array}{l}\text { Swage } \\
\text { Compaction }\end{array}$ & $\begin{array}{l}7.180 \\
(9,425)\end{array}$ & 10,550 & $\begin{array}{l}430 \\
(14.0)\end{array}$ & $\begin{array}{c}167 \\
(50.0)\end{array}$ & Failed \\
\hline $\begin{array}{l}\text { Swage } \\
\text { Compaction }\end{array}$ & $\begin{array}{l}5,470 \\
(7,800)\end{array}$ & 8,050 & $\begin{array}{l}426 \\
(13.9)\end{array}$ & $\begin{array}{c}165 \\
(46.21\end{array}$ & Failed \\
\hline $\begin{array}{l}\text { Swage } \\
\text { Compaction }\end{array}$ & $\begin{array}{l}5,260 \\
(7,800)\end{array}$ & 7,750 & $\begin{array}{l}430 \\
(14.0)\end{array}$ & $\begin{array}{c}167 \\
(44.4)\end{array}$ & Out of Core \\
\hline $\begin{array}{l}\text { Swage } \\
\text { Compaction }\end{array}$ & $\begin{array}{l}5,890 \\
(7,800)\end{array}$ & 8.650 & $\begin{array}{l}430 \\
(14.0)\end{array}$ & $\begin{array}{c}167 \\
(49.8)\end{array}$ & Out of Core \\
\hline $\begin{array}{l}\text { Swage Over } \\
\text { Ground Pellets }\end{array}$ & $\begin{array}{l}10,5 \dot{5} 90 \\
(19,500)\end{array}$ & 14,800 & $\begin{array}{c}342 \\
(11.1)\end{array}$ & $\begin{array}{c}133 \\
(35.7)\end{array}$ & Out of Core \\
\hline $\begin{array}{l}\text { Swage Over } \\
\text { Ground Pellets }\end{array}$ & $\begin{array}{l}10,850 \\
(19,500)\end{array}$ & 15,200 & $\begin{array}{c}338 \\
(11.0)\end{array}$ & $\begin{array}{c}131 \\
(36.5)\end{array}$ & Out of Core \\
\hline $\begin{array}{l}\text { Vibratory } \\
\text { Compaction }\end{array}$ & $\begin{array}{l}7.430 \\
(7,725)\end{array}$ & 10,900 & $\begin{array}{c}348 \\
(11.4)\end{array}$ & $\begin{array}{r}135 \\
(47.8)\end{array}$ & $\begin{array}{l}\text { Out of Core } \\
\text { (Suspect) }\end{array}$ \\
\hline $\begin{array}{l}\text { Vibratory } \\
\text { Compaction }\end{array}$ & $\begin{array}{l}7,280 \\
(7,725)\end{array}$ & 10,700 & $\begin{array}{c}315 \\
(10.3)\end{array}$ & $\begin{array}{c}122 \\
(46.9)\end{array}$ & $\begin{array}{l}\text { Out of Core } \\
\text { (Failed) }\end{array}$ \\
\hline $\begin{array}{l}\text { Vibratory } \\
\text { Compaotion }\end{array}$ & $\begin{array}{c}7,290 \\
(7,726)\end{array}$ & 10,700 & $\begin{array}{c}315 \\
(10.3)\end{array}$ & $\begin{array}{c}122 \\
146.91\end{array}$ & $\begin{array}{l}\text { Out of Core } \\
\text { (Suspect) }\end{array}$ \\
\hline
\end{tabular}

Each developmental assembly contains 121 fuel rods:

109 fuel rods are 0.425 inch in diameter by 6 feet long, and 12 corner fuel rods are 0.320

Inch in diameter by of ret luny.
Group I Design Lifetime (Averaye Assembly Burnup) - 10,000 MWa/Tu

(b) Groups I and II Design Lifetime (Average Assembly Burnup - 15,000 MWu/TU 
Table 2

INTERIM EXAMINATION SUMMARY

CONSUMERS DEVELOPMENTAL FUEL

May $1964^{16}$

July-Sept.
1964

1964

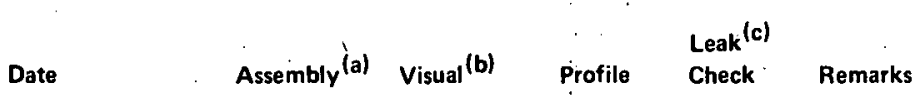

PE 1.3 . . P

PO 2-4 P

PE 1.4

PO 1.4 P

D. 1.3

PO.2

D -2

$\because$

D 5, 6 P $\quad$ D -5

$\begin{array}{lll}D 8,9 & P & D-8\end{array}$

D 10-12 P

April 17

1965

PE - 3

PO 2

P Yes

D -1

$P$.

Yes

Yes'

April 18

1966

PE $-3(T)$

PO. 2 (T)

D. 2

$\mathbf{P}$

$\mathbf{P}$

$\mathbf{P}$

D $\cdot 5$

P

D - 8

D -11

D -17

D -19

October $^{19}$

PO 1,3,4

D 1:-3

D 4-6

D 10 (T)

TV

D 11 (T)

D 12 (T)

D 13 (T)

D 14 (T)

D $15(T)$

$D 18(T)$

D 19 (T)

D $20(T)$

B .

P. TV

TV

TV

TV

p

P

P. TV

Yes

Rods from

January
1967

D. 2

D -19

May $1967^{20}$

PO: 1,3

D - 1-3

D. $4(T)$

D $\cdot 5,6$

D.2, P

D $\cdot 7.9$

D-5, P

D $-16,17$

D-8, P

D-17, P

$D \cdot 2(T)$

$\mathbf{P}$

February

1968 .

(a) $\mathbf{T}=$ Irradiation terminated at this inspection.

(b) $\mathrm{P}=$ Periscope; $\mathrm{B}=$ Binoculars; TV = Closed-Circuit Tetevision.

(c) Leak check is by isolating individual fuel bundies and monitoring for radioactivity in the isolation chamber. A positive signal $(X)$ indicates fission product release. $(0)$ indicates low radioactivity signal.
Little deposit; circumferential striations on PE bundles.

Circumferential Striations.

Circumferential striations.

Bambooing, streaming. Pelletspaced circumimferential ridges and 6-mil ovality measured.

Circumferential striations

Deposit spalling.

Uniform deposit.

Wire brushing removed striated appearance, but did not change profiles. . $\therefore$.

Split cladding observed. .

Suspect area noted.

Up to 7-mil diameter increase

caused by deposits.

Deposit spalling noted on profile traces.

Deposits evident on profiles.

D-2 fuel rods exchanged for later profiling.

Split cladding observed for D-10 through 0.13.

No visible failures for $D-14,-15$.

No visible failures in D-18, -20 .

Some diameter reduction on one rod since April 1966.

Little change since April 1966.

No visible failures.

Gross deposit spalling since

April 1966:per profiles.

Deposit spalling.

No defects visible.

$$
\text { a. }
$$




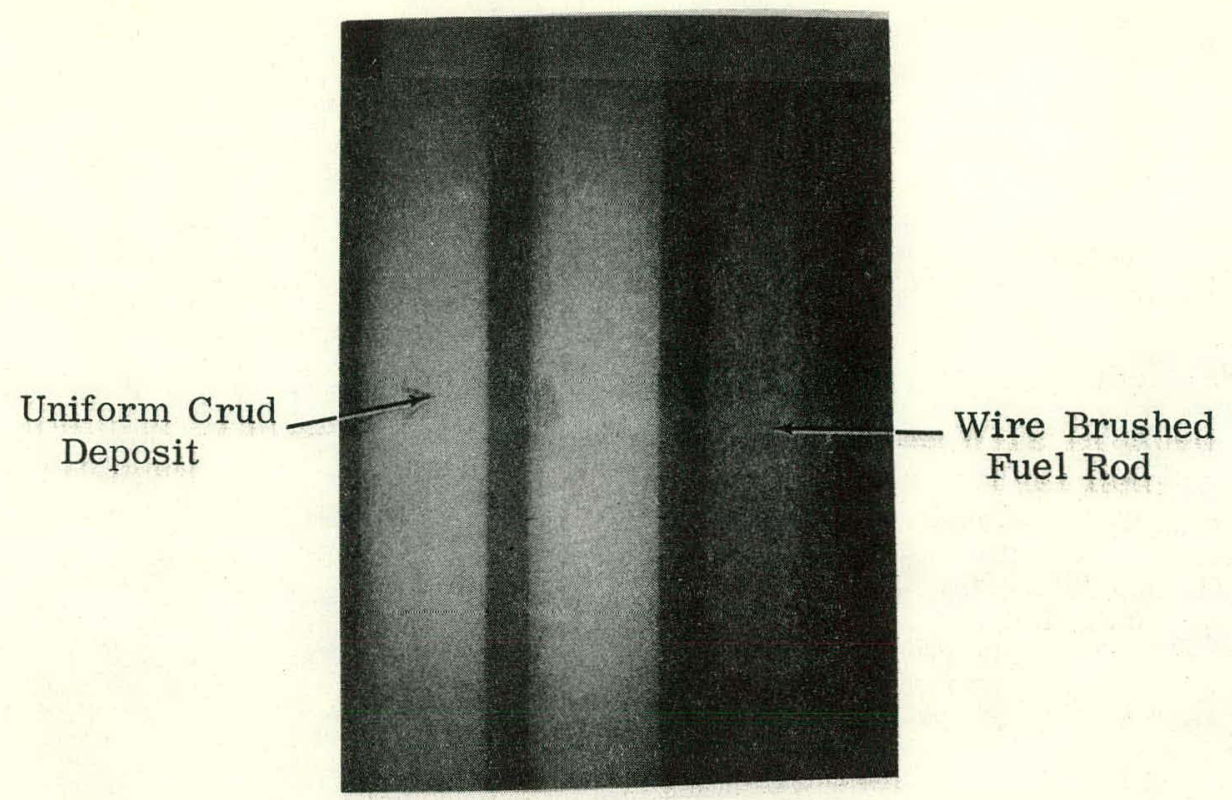

Figure 3A. Assembly D-2-Fuel Rods Away fram Spacer

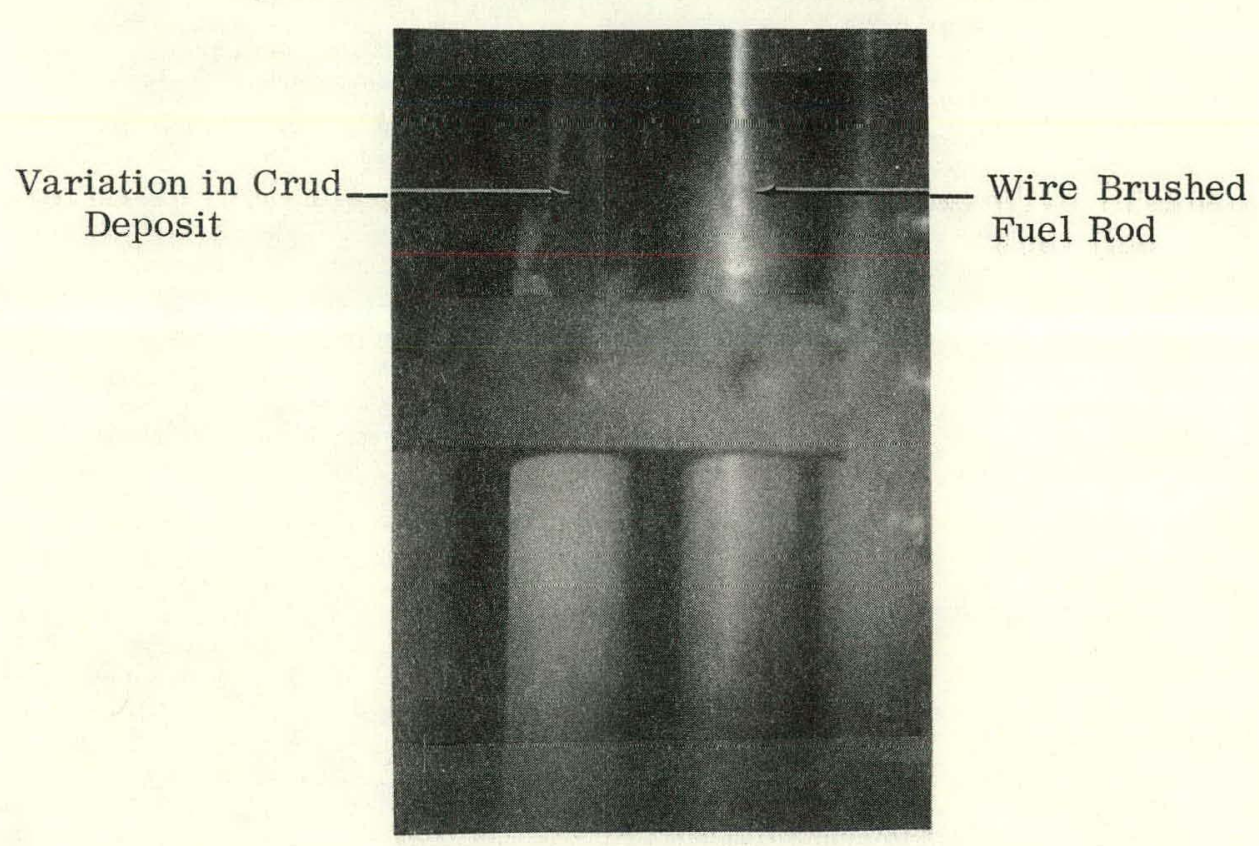

Figure 3B. Assembly D-2-Fuel Rods at Spacer

Zircaloy-2 Cladding, 0.030-inch Wall

$\mathrm{UO}_{2}$ Pellet Fuel, 0.007-inch Initial Diametral Pellet-to-Cladding Gap Burnup: $6410 \mathrm{MWd} / \mathrm{TU}$ Average

Peak Fuel Rod Surface Heat Flux: $355,000 \mathrm{Btu} / \mathrm{h}-\mathrm{ft}^{2}$ 

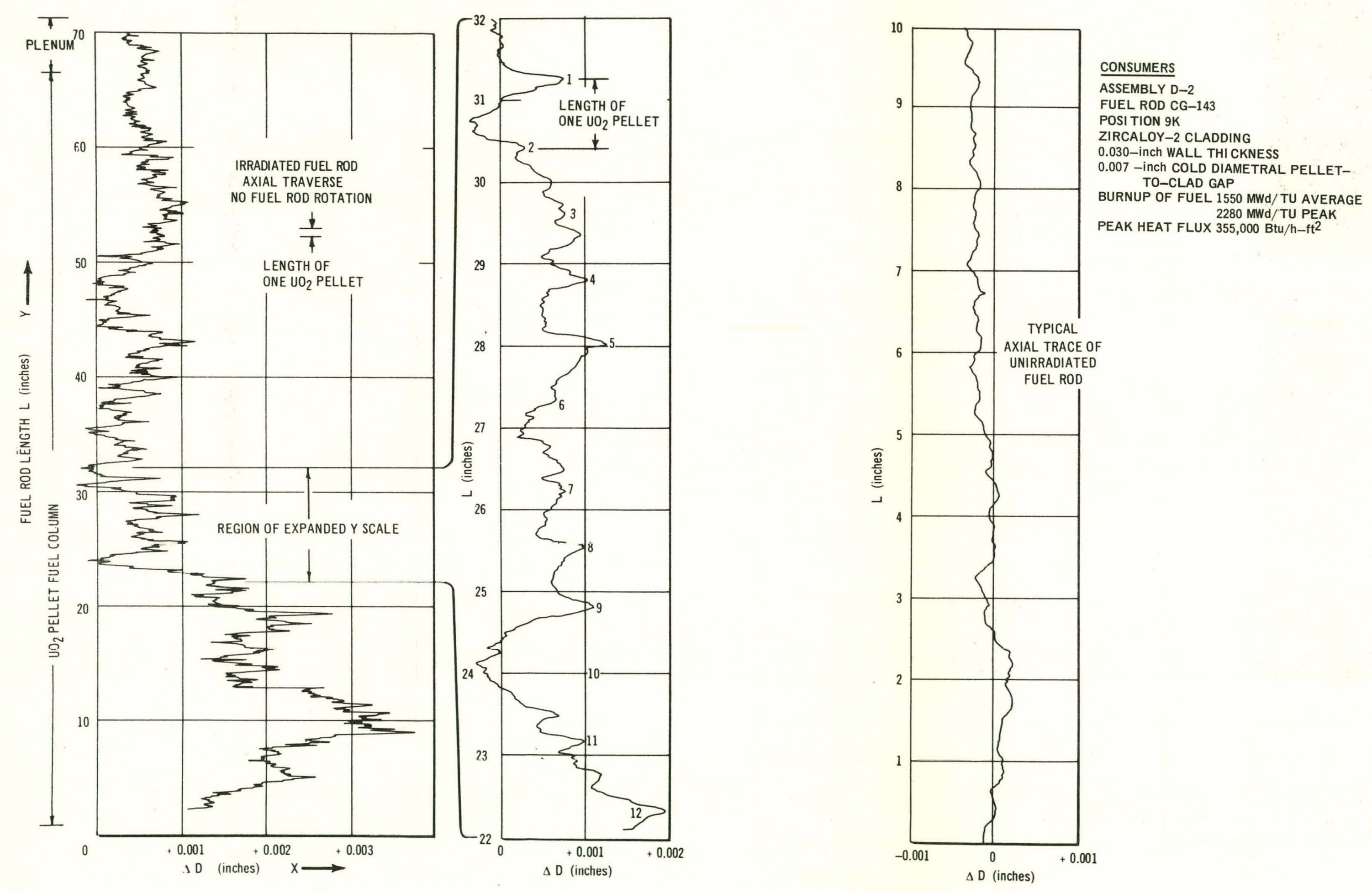

Figure 4. Profile Traces, $\triangle D$ versus $L$, of Irradiated Fuel 


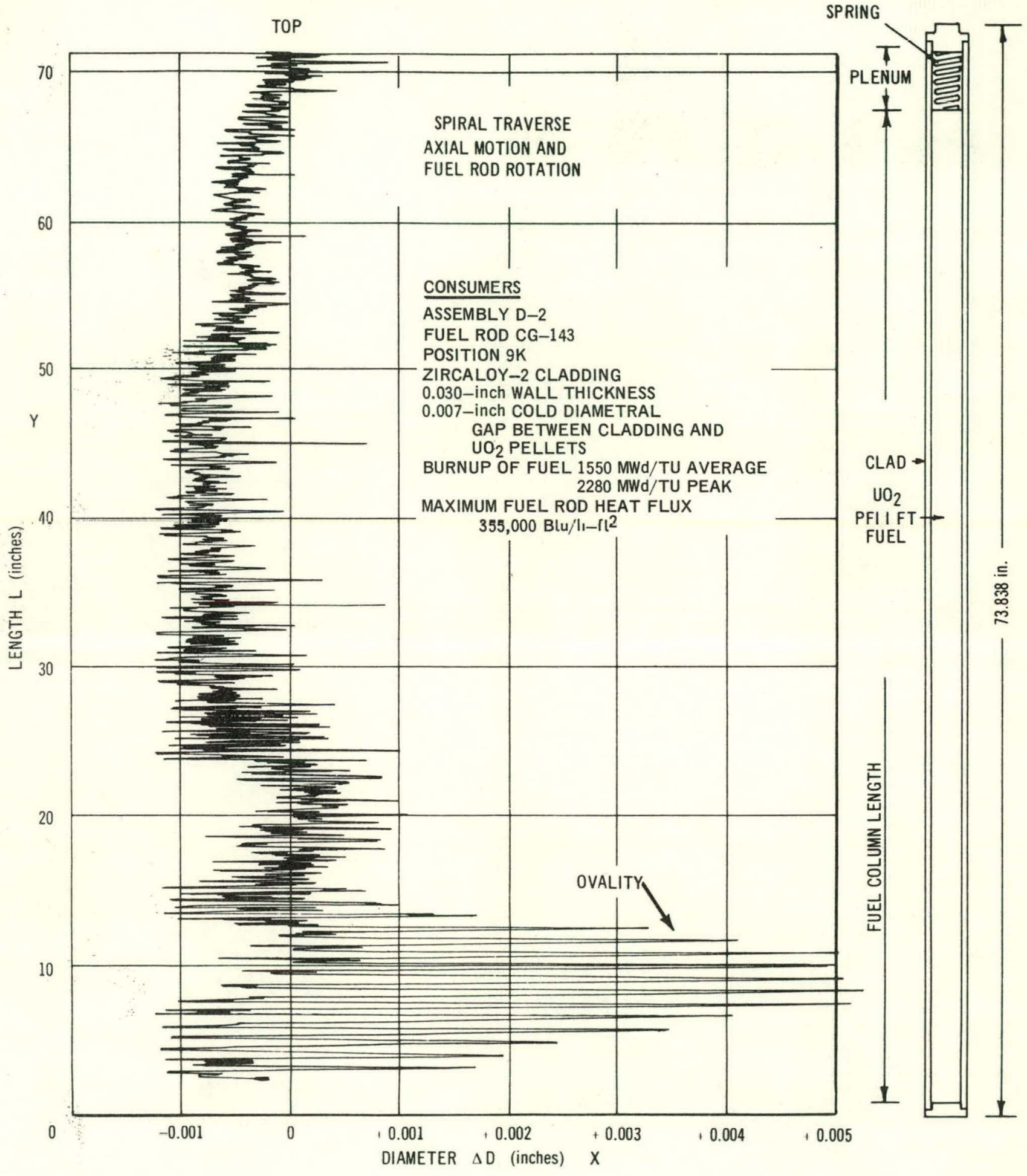

Figure 5. Profile Trace, $\Delta D$ versus $L$, of Freestanding Irradiated Fuel Rod from Assembly D-2 Showing Local Ovality of Cladding 

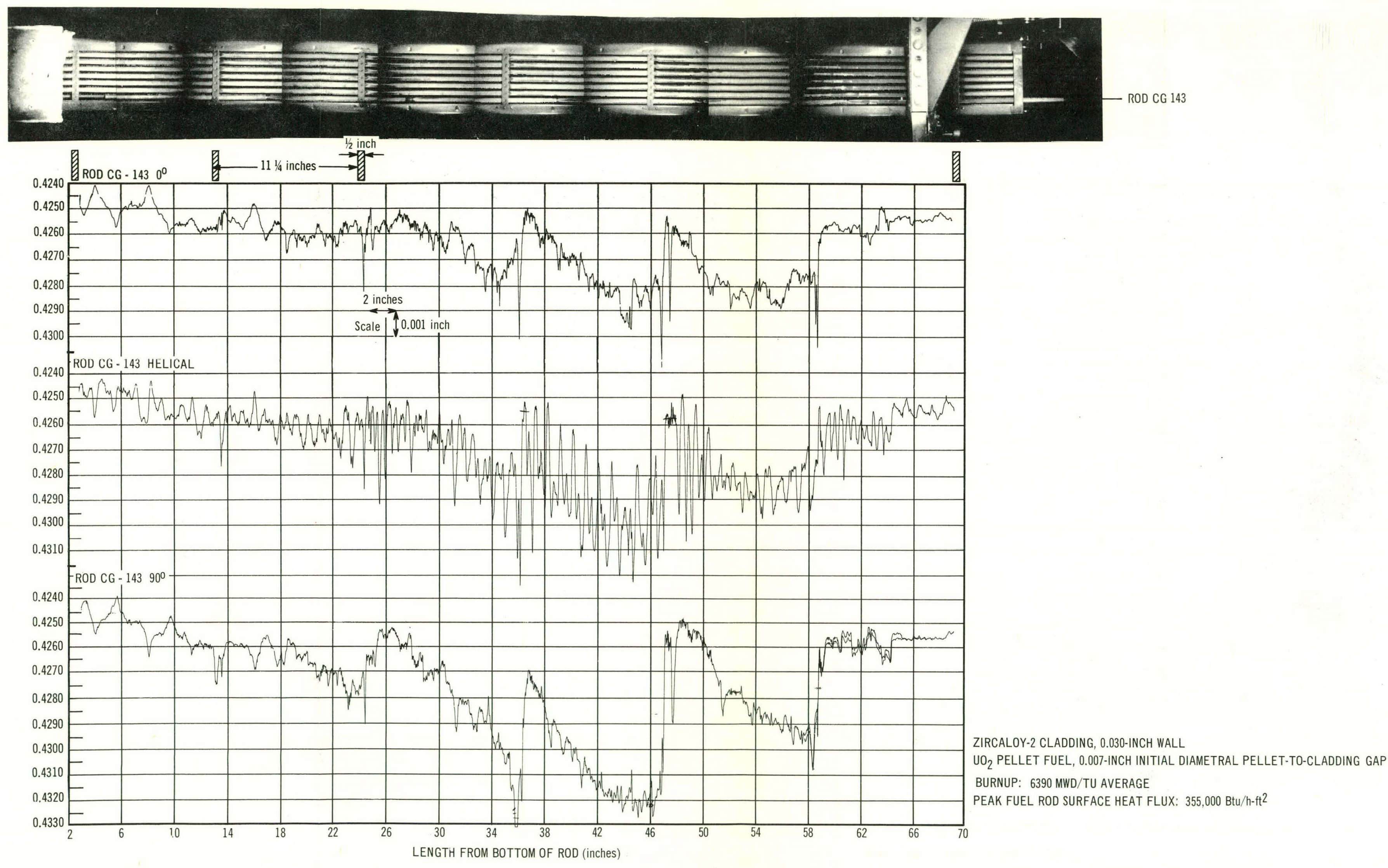


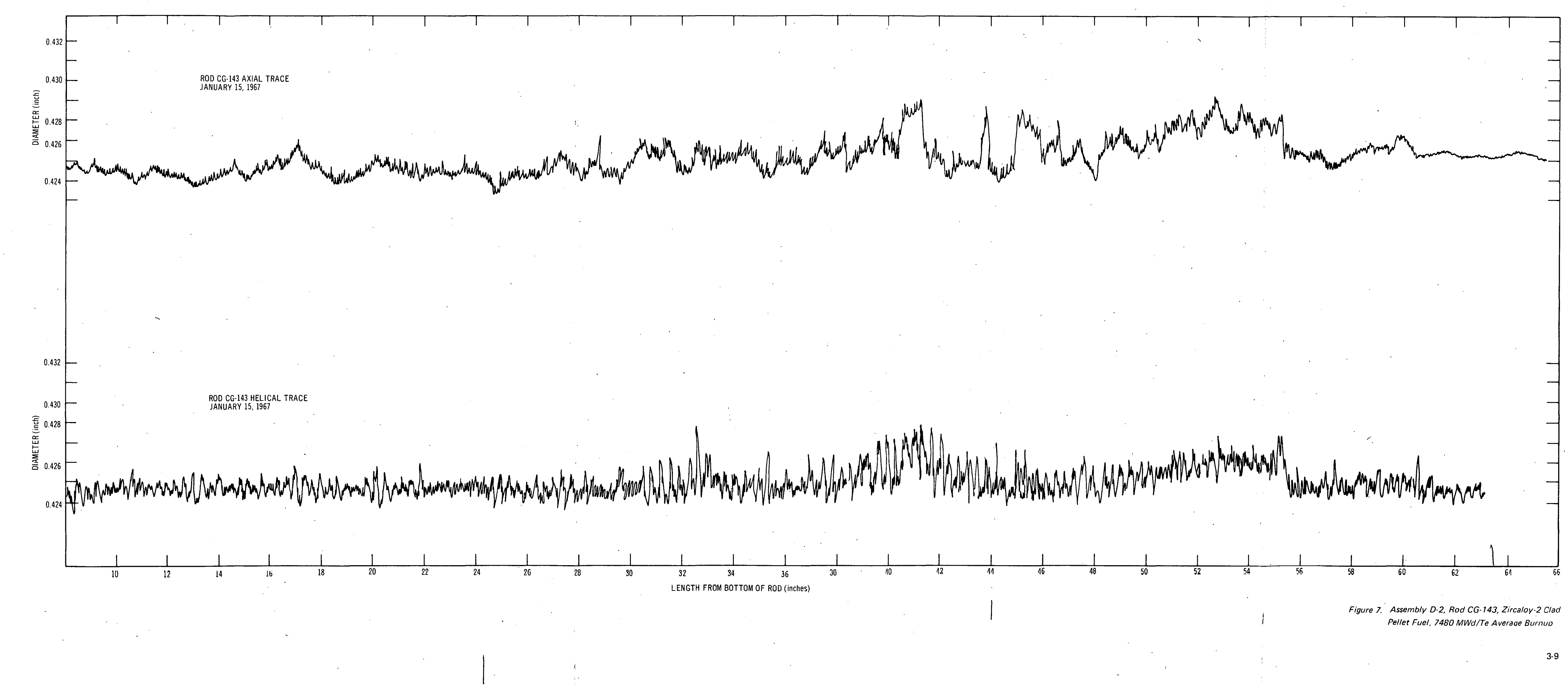




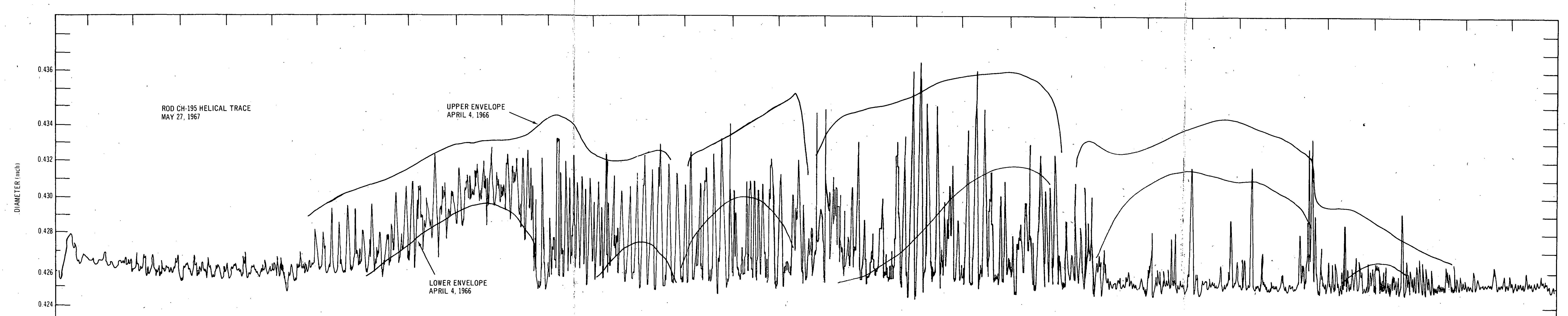


The profilometer measurements indicated that (a) the deposits were heaviest on peripheral rods in the assemblies, (b) there was more deposit on the side of peripheral rods facing the channel, (c) the spacer regions of the rods were free of deposit except for 10- to 20 - mil high by $\sim 1 / 4$-inch diameter spots at or near spacer band dimples, ${ }^{*}$ and (d) the axial distribution of the deposits was similar to the axial power distribution.

Thus, it appears that the major influence on deposit location and thickness was the coolant hydraulic variations caused by spacers and channel, and a secondary consideration was the local heat flux.

\subsubsection{Post-Irradiation Examination of Unfailed Rods}

Several developmental fuel rods were shipped to the Vallecitos Nuclęar Center for post-irradiation examination. To ship the fuel rods from the Big Rock Point reactor to the Vallecitos Radioactive Materials Laboratory (RML) hot cells, it was necessary to puncture and shear the rods into two sections each so they would be short enough to be handled at RML. Table 3 lists the developmental fuel rods shipped to RML and summarizes the post-irradiation examination. The failed fuel rods are discussed in Section 4. The examination is described in detail in Reference 21.

Visual examination of the fuel rods in the hot cells revealed irregular reddish-brown to black coloring. Zircaloy-clad rod CG-143 had white oxide spots over much of the cladding surface. Localized deposits were observed on several rods (Figure 9). Cladding wear at spacers was generally small. The greatest amount of wear observed, estimated at 2 to 3 mils, is shown in Figure 10.

One metallographic cross section was made of the Zircaloy-2 clad, pellet fuel rod CG-143 in order to verify the prnfilometer meacuromonts of deposits uı lle claddlng and tó observe cladding conditions under the deposits. Figure 11 shows this cross section. Figure 12 shows the typical cladding condition. The deposit has a very high density, is adherent, and extends around 180 degrees of circumference, with a maximum thickness of 3.5 mils. Figure 13 shows the detailed deposit appearance. Between the deposit and cladding is an oxide layer 0.1 mil to 0.2 mil thick. This oxide layer extends completely around the circumference and locally thickens to 3 mils on the portion of the circumference with least deposit (see Figure 14). The thicker areas correspond to the white spots observed visually. The cladding was locally thinned about 2 mils $(7 \%)$ at the oxide spots. There was no evidence of cladding overheating or deterioration at either the oxide or deposit region.

The main interest in the pellet-Zircaloy rods was the cladding performance in areas with relatively heavy deposits. The visual examination and the metallographic section both indicate satisfactory performance for up to $7500 \mathrm{MWd} / \mathrm{T}$ average exposure. However, if deposits continue to build up without spalling off, the cladding could eventually overheat. As noted above, profile measurements of rod CG-143 suggest that spalling is actually taking place. If this is the case, the deposit thickness and resulting temperature increase may be self limiting.

One apparent beneficial effect of the deposits is the absence of localized heavy-oxide spots under them (assuming that such oxide spots are potentially detrimental, for example, eventually thinning the cladding locally so that failure results).

It should be noted that all of the foregoing discussion and observations on deposition are specific for the past BRP situation where the feedwater heaters contained Admiralty metal and which contributed large quantities of copper and zinc to the primary coolant which subsequently deposited on the fuel rods. The feedwater heaters have recently been changed to stainless steel, so future deposits should be different in composition and are expected to be much lower in magnitude.

- The deposit spots were also observed visually during hot cell examinations. See Figure 9. 
Table 3

CONSUMERS R\&D FUEL ROD SHIPMENT AND HOT CELL EXAMINATION SUMMARY

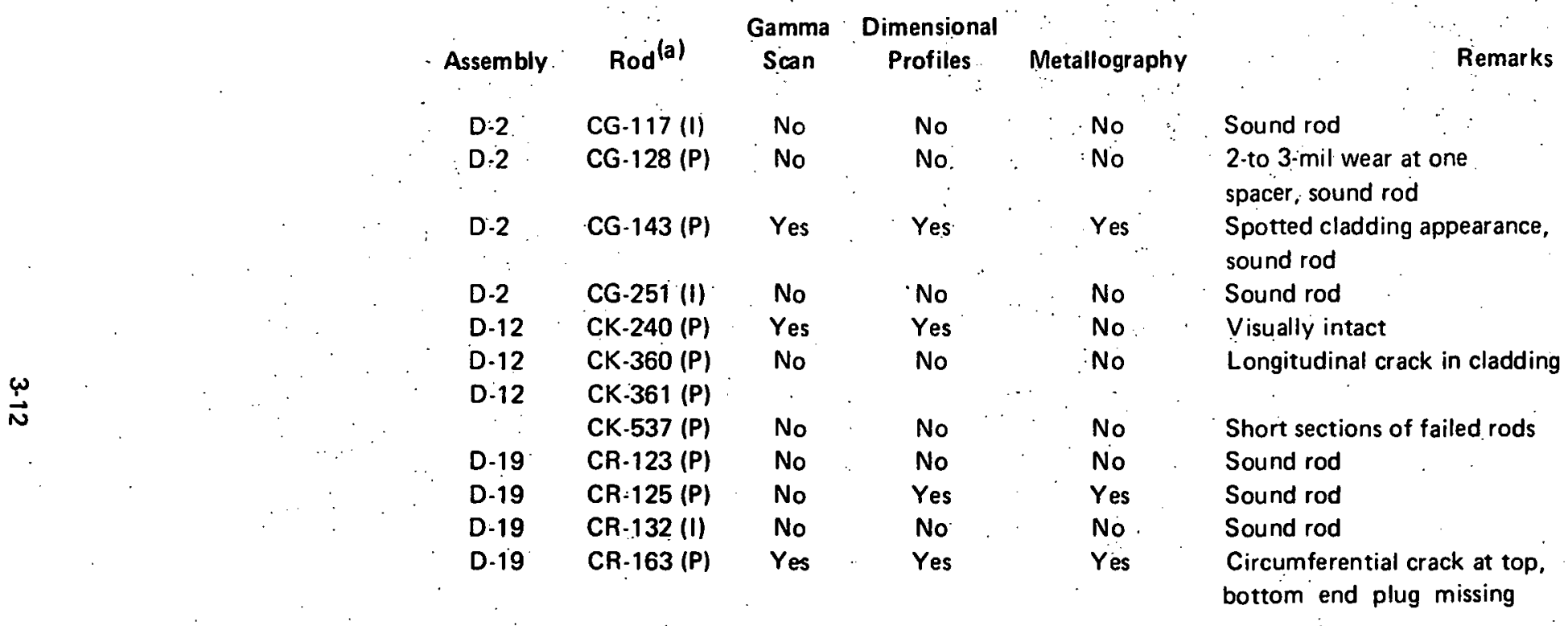

(a) $\quad(P)=$ peripheral rod in assembly, (I) $=$ interior rod. Peripheral rods generaliy operated at 10 to $15 \%$ higher power than interior rods.

(b) All fuel rods were visually examined.

(c) The four $C R$ rods were bubble leak checked at 20 psi. Only CR-163.emitted bubbles.

(d) CR-125 and CR-163 were neutron radiographed. CR-163 showed a dark spot (cladding (cladding hydriding) at top crack. 


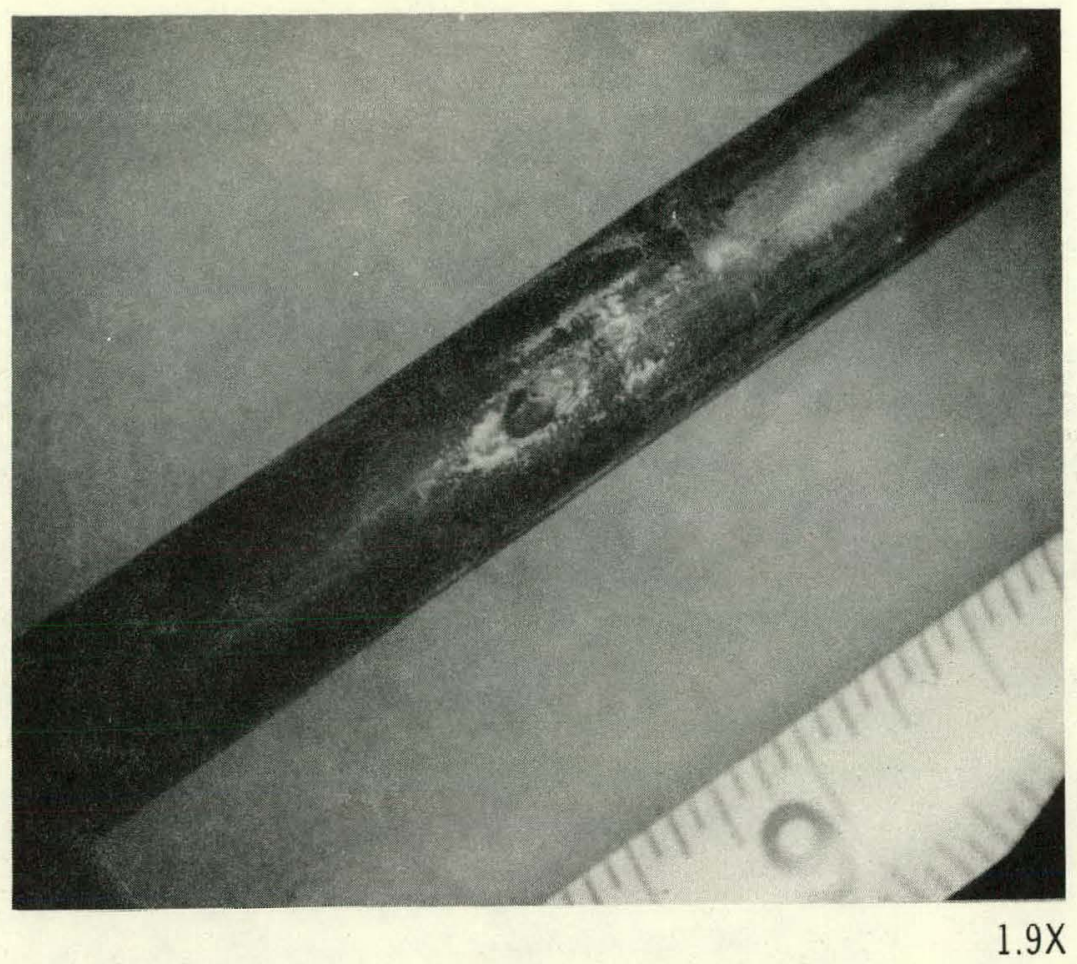

Figure 9. Localized Deposit Buildup at a Spacer (Part of the Deposit Has Chipped Away), Zircaloy-2 Clad Powder Fuel, Rod CR-163

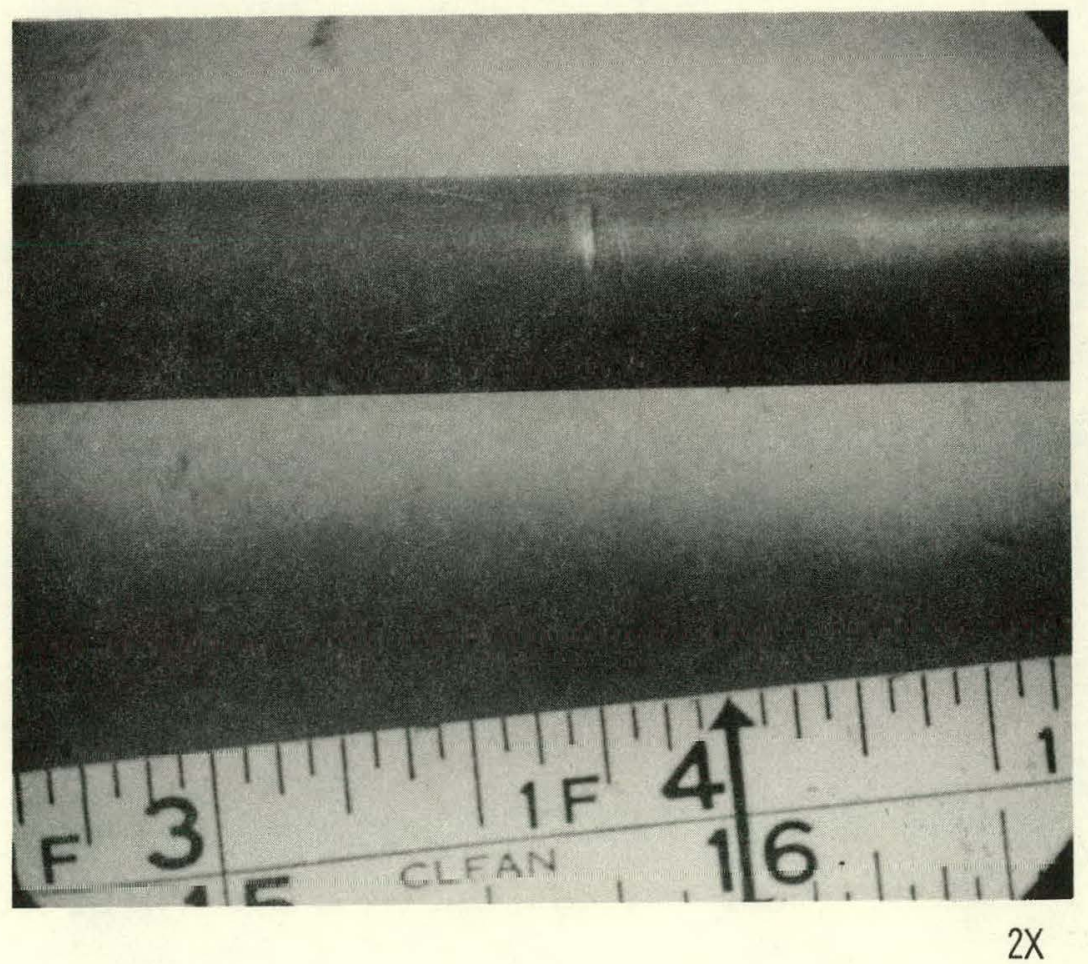

Figure 10. Wear at Spacer Wire Contact Point (No Springs in Spacer), Zircaloy-2 Clad Pellet Fuel, Rod CG-128 


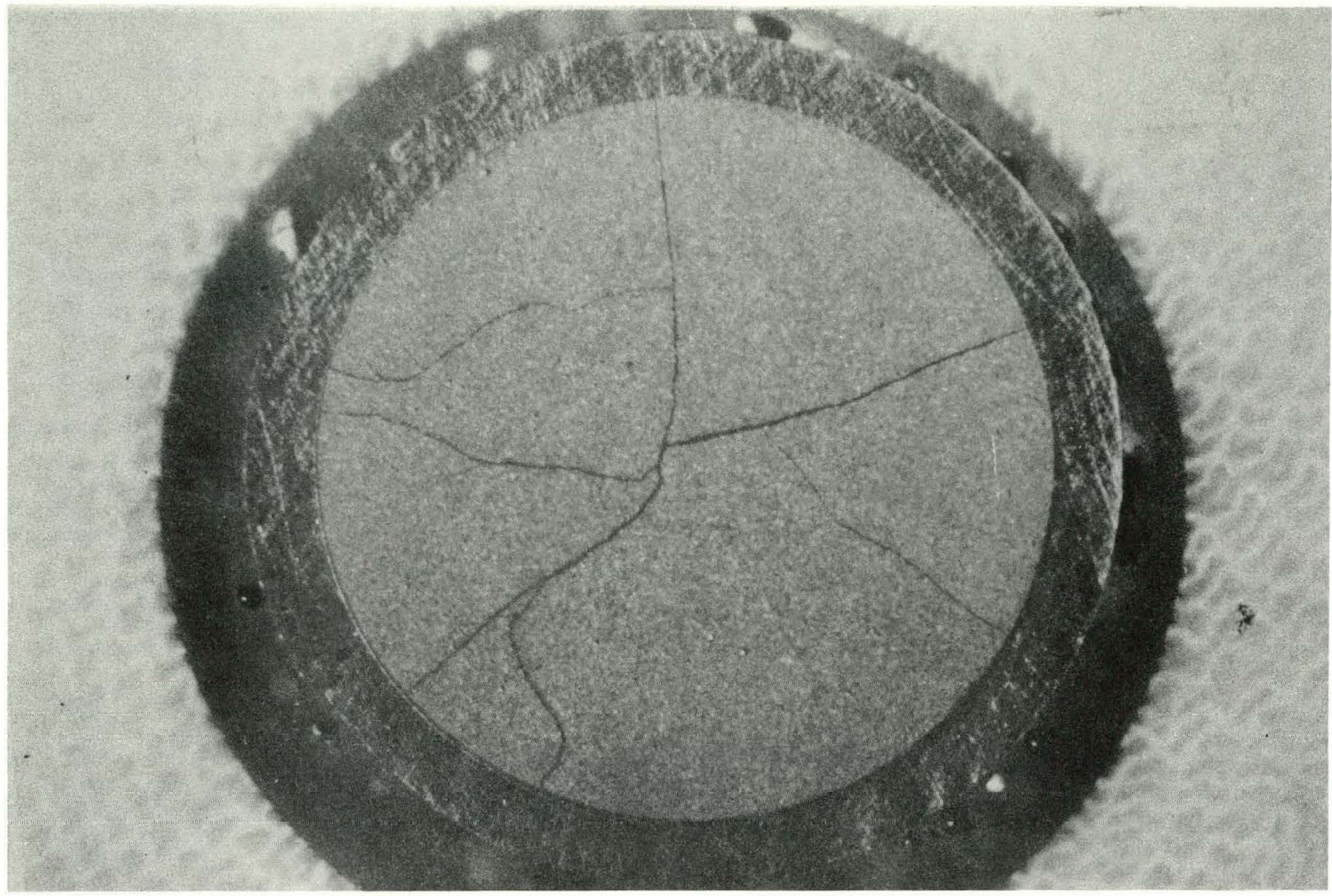

AS GROUND

Figure 11. Fuel and Cladding Cross Section of Fuel Rod CG-143

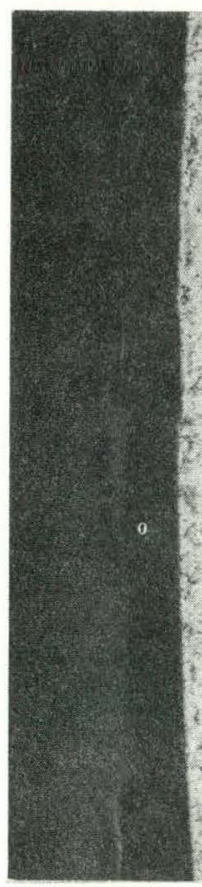

ETCHED

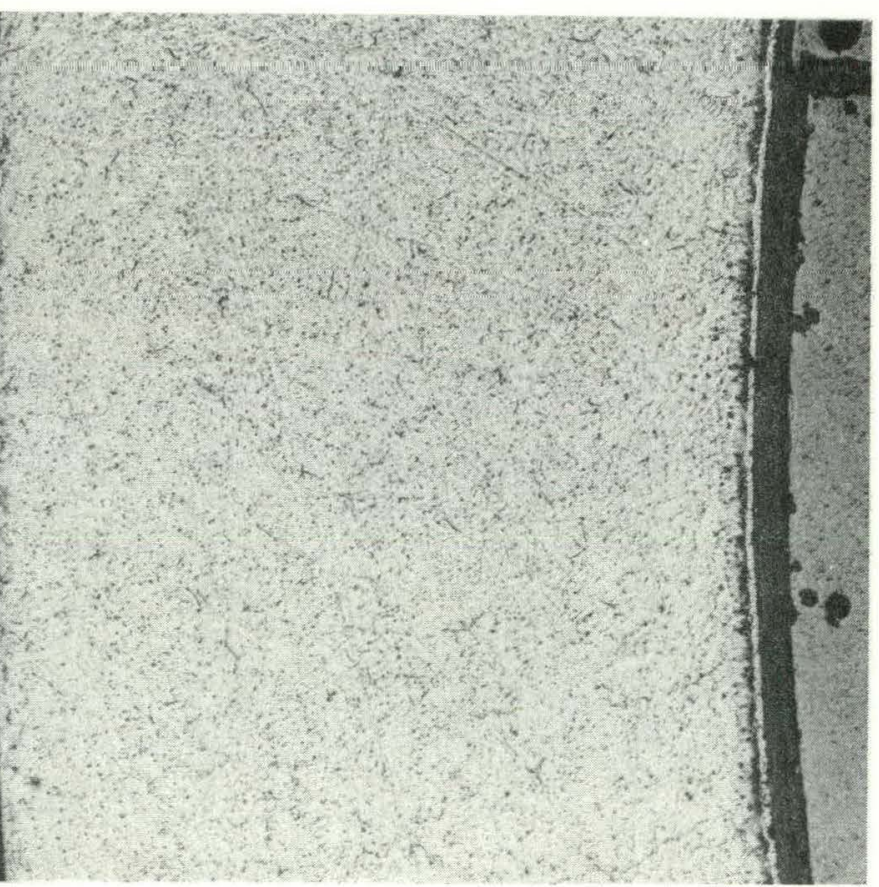

$100 x$

Figure 12. Typical Cladding of Fuel Rod CG-143 

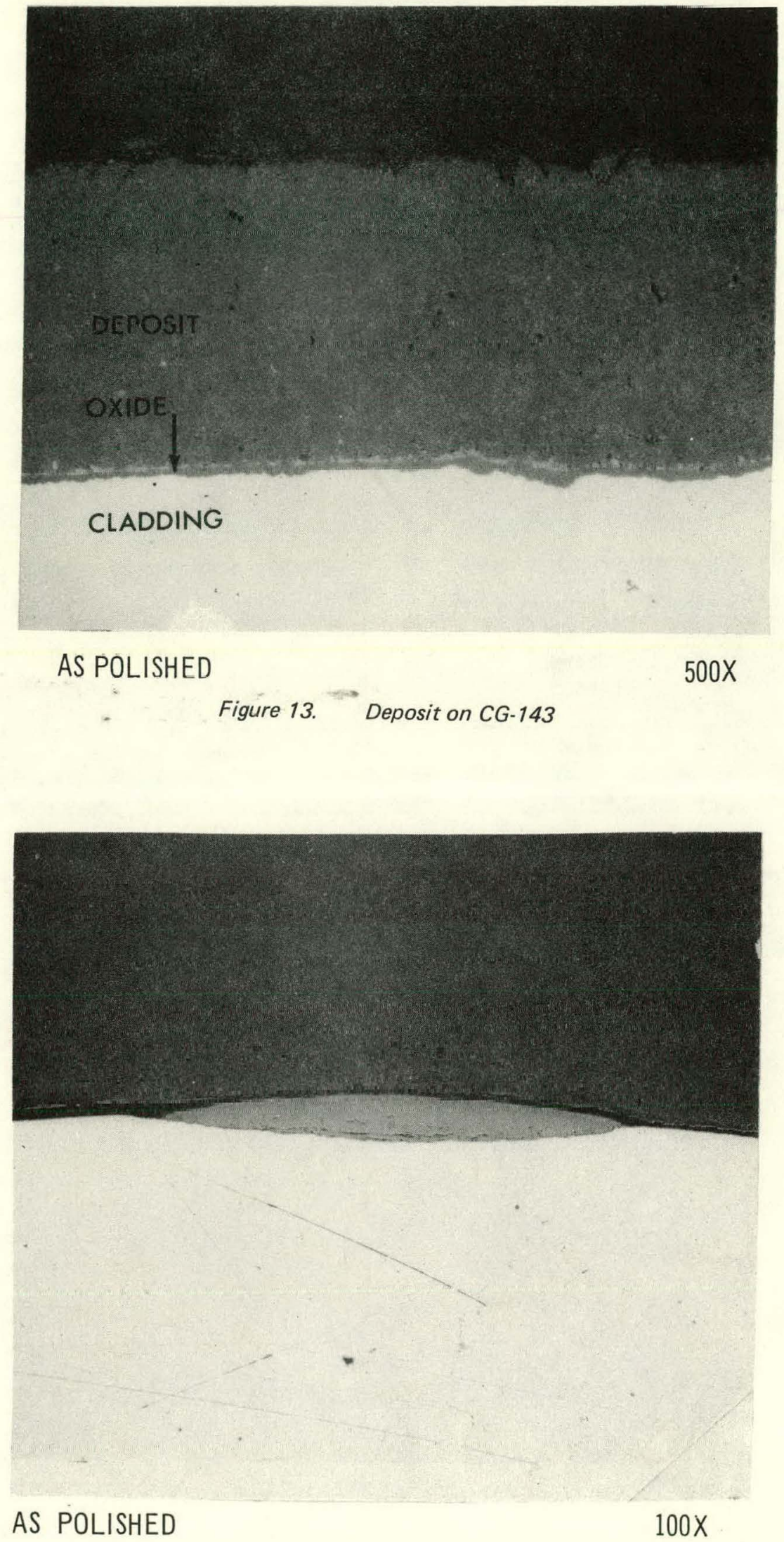

Figure 14. Oxide Spot on CG-143 


\section{FAILURE DESCRIPTIONS AND ANALYSIS}

The objectives of the post-irradiation failure examinations were to document the condition of failed fuel assemblies and individual developmental fuel rods, and to establish the failure mechanisms encountered with Type-304L stainless steel, Zircaloy-2, and Incoloy-800 clad fuel rods containing $\mathrm{UO}_{2}$ pellet or powder fuel.

The failed assemblies were detected by a combination of fuel assembly leak-testing and visual examination in the Consumers Big Rock Point fuel pool.

In addition to these initial observations obtained in the fuel pool, selected fuel rods were cut in half and shipped to the Vallecitos hot cells for detailed post-irradiation examination and failure analysis.

The following subsections describe the failures in detail:

4.1. Type-304L Clad Fuel Rods (Failed Assemblies PE-3 and PO-2)

4.2. Incoloy-800 Clad Fuel Rods (Failed Assemblies D-4, -10, -11, -12, and -13)

4.3. Zircaloy-2 $\mathrm{Clad} \mathrm{UO}_{2}$ Powder Fuel Rods (Failed Assemblies D-18, -19, and -20)

4.4. Zircaloy-2 $\mathrm{Clad} \mathrm{UO}_{2}$ Pellet Fuel Rods (Failed Assembly D-2)

\subsection{TYPE 304-L CLAD FUEL RODS}

Starting in September 1965, an increase in the fission product activity levels was indicated by the reactor off-gas monitors, indicating that fuel rod failures were occurring in the Big Rock Point reactor. Failures similar to those which occurred in the VBWR due to stress-assisted intergranular corrosion of stainless steel clad fuel rods were suspected. ${ }^{22}$

\subsubsection{Visual Inspection of Developmental Assemblies Containing Stainless Steel Clad Fuel Rods During the April 1966 Shutdown}

During the outage, visual inspections were performed on selected irradiated developmental fuel assemblies, using an underwater periscope in the Consumers spent fuel pool.

Two of the eight Group I developmental assemblies contained failed fuel rods: PE-3 (Type-304L stainless steel cladding, collapsed 0.010-inch-wall, swaged-over-pellet $\mathrm{UO}_{2}$ ) and PO-2 (Type 304L stainless steel cladding, collapsed 0.010 -inch wall, swage compacted powder $\cup_{2}$ ).

\section{a. Assembly PE-3}

This assembly contained fuel rods clad with 0.010 -inch-wall, collapsed Type-304 stainless steel initially in an annealed condition. The fuel rods were fabricated by swaging the thin cladding down over $\mathrm{UO}_{2}$ pellet fuel. When examined, this assembly had accumulated $7500 \mathrm{MWd} / \mathrm{TU}$ average fuel burnup, and operated at a maximum fuel rod surface heat flux of $366,000 \mathrm{Btu} / \mathrm{h}-\mathrm{ft}^{2}$.

Visual inspection of the PE-3 assembly revealed that at least five fuel rod failures had occurred. Two peripheral fuel rods and three interior fuel rods were verified as being failed. The visual appearance of the failed regions of the fuel rods was similar to the Type-304 stainless steel cladding failures in the VBWR, ${ }^{22}$ and is attributed to stress-assisted intergranular corrosion of the Type-304L stainless steel fuel cladding. The appearance of the peripheral fuel rod failures is shown in Figure 15.

b. Assembly PQ-2

This assembly contained fuel rods clad with 0.010-inch-wall, collapsed Type-304 stainless steel in a cold-worked condition. The fuel rods were fabricated by swage compaction of arc-fused $\mathrm{UO}_{2}$ powder. This assembly had accumulated $7680 \mathrm{MWd} / \mathrm{IU}$ average fuel burnup prior to the inspection, and had operated at maximum fuel rod surface heat fluxes of $384,000 \mathrm{Btu} / \mathrm{h}-\mathrm{ft}^{2}$. 


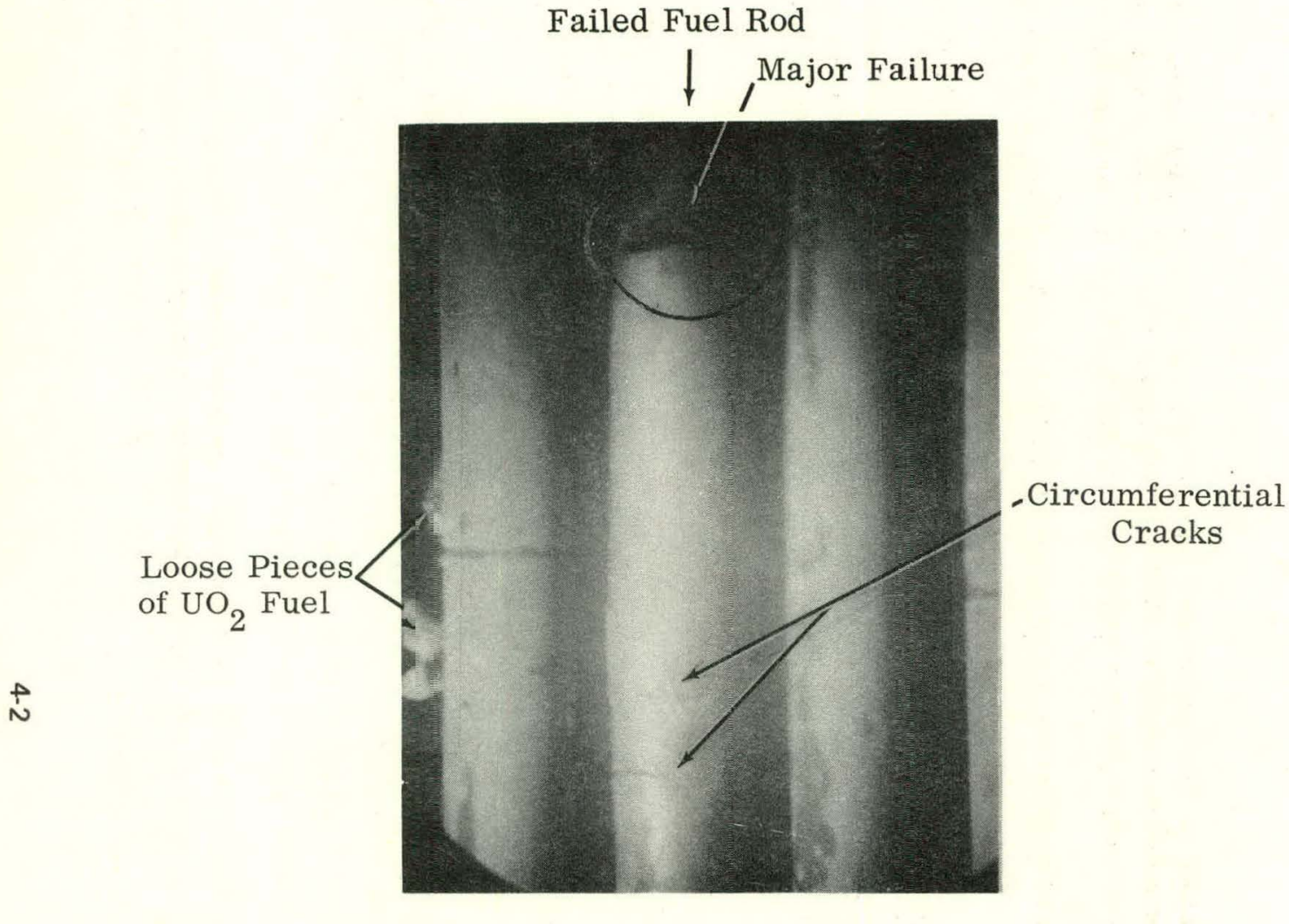

a. Region of Multiple Circumferential Cladding Cracks

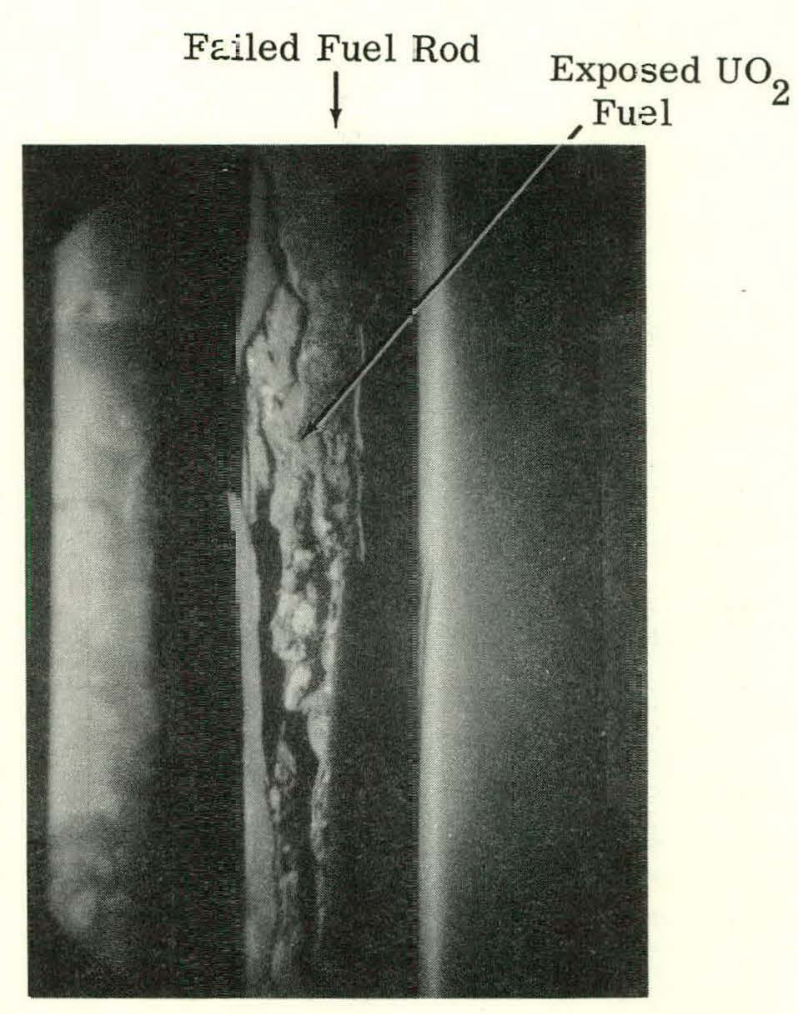

b. Fai'ure Region Showing Loss of Clatding and Exposed $\mathrm{UO}_{2}$ Fuel

Type-304L Stainless Steel Cladding, Collapsed 0.010-inch ${ }^{-1} \equiv l l$

Cladding Swaged Down Over $\mathrm{UO}_{2}$ Pellet Fuel

Burnup: $7500 \mathrm{MWd}$.'TU Average

Peak Fuel Rod Surface Heat Flux: $366,000 \mathrm{Btu} / \mathrm{h}-\mathrm{ft}^{2}$ 
Visual inspection of the PO-2 assembly revealed a minor suspect region on one fuel rod. Penetration of the cladding in this one area could not be verified by the visual examination. No gross fuel rod failures comparable to those observed in the PE-3 assembly occurred in the PO-2 assembly. The appearance of the suspect region on the PO-2 assembly is shown in Figure 16.

The PE-3 and PO-2 assemblies contained fuel rods clad with Type-304L stainless steel of identical chemistry (same heat of material), and were subjected to similar operating parameters such as cladding temperature, fuel rod surface heat flux and power level, fuel burnup, and number of cycles, and to the same coolant environment during irradiation in the Big Rock Point reactor. These similarities and the number of fuel rods irradiated (121 fuel rods in each assembly-109 fuel rods are 0.425 inch in diameter, and 12 corner fuel rods are 0.320 inch in diameter) lead to the conclusion that the potential performance of $\mathrm{UO}_{2}$ powder fuel is better than $\mathrm{UO}_{2}$ pellet fuel in fuel rods clad with collapsed Type-304L stainless steel.

The lack of multiple fuel rod failures in the PO-2 assembly is attributed to lower local cladding stresses ${ }^{23}$ during operation which delayed the initiation of stress assisted intergranular corrosion of the cladding. In the case of the PE-3 assembly, high local cladding stresses, which result in plastic deformation and circumferential ridge formation during operation, ${ }^{23}$ are known to exist at $\mathrm{UO}_{2}$ pellet interfaces. The failure of the PE-3 assembly fuel rods is attributed to stress-assisted intergranular corrosion at regions which have high localized stresses.

As a result of these inspections, the irradiation of the PE-3 and PO-2 assemblies was terminated.

\subsection{INCOLOY-800 CLAD FUEL RODS (FAILED ASSEMBLIES D-4, -10,-11, -12, and -13)}

During reactor operation prior to the September 1966 outage, rapid increase in plant off-gas activity indicated that multiple fuel failures were occlrring. Fission gas leak checks of individual assemblies during the outage indicated assemblies D-10, $-11,-12$, and -13 contained failed fuel rods.

\subsubsection{Inspection of Incoloy-800 Clad Fuel Rods in Developmental Assemblies During Reactor Shutdowns 1966 and May 1967}

Visual inspections were performed using underwater closed-circuit television equipment and a periscope in the Consumers spent fuel pool. The exposures attained prior to the shutdown by the various assemblies which were inspected are summarized in Table 4.

\section{a. Assembly D-4}

This assembly contained fuel rods clad with 0.019 -inch-wall, freestanding Incoloy-800 in the annealed condition. The fuel rods were fabricated by loading centerless-ground $\mathrm{UO}_{2}$ pellets into weld-drawn Incoloy-800 tubing with an initial pellet-to-cladding gap of 0.006 inch. The September-October 1966 examination of the assembly after an average exposure of $6930 \mathrm{MWd} / \mathrm{TU}$ at fuel rod surface heat flux peaks of up to $342,000 \mathrm{Btu} / \mathrm{h}-\mathrm{ft}^{2}$ revealed circumferential striations at pellet interfaces.

The striations were most pronounced at the axial peak heat flux region of the fuel rods, but were not as distinct as those observed previously ${ }^{1,2}$ on assembly D-5, which is similar. No fuel rod failures were observed at this inspection.

During the May-June 1967 outage, examination of assembly D-4 after an average exposure of 9910 $\mathrm{MWd} / \mathrm{TU}$ revealed variation in deposit patterns similar to those described above. The deposits appear to have spalled or chipped in several areas. No fuel rod failures were observed. However, fission product activity measurements (leak test) indicated that assembly D-4 contained failed fuel rods. As a result, assembly D-4 was deleted from the next core loading. 
Table 4

DEVELOPMENTAL FUEL ASSEMBLIES CONTAINING INCOLOY-800 CLAD FUEL RODS INSPECTED DURING REACTOR SHUTDOWNS

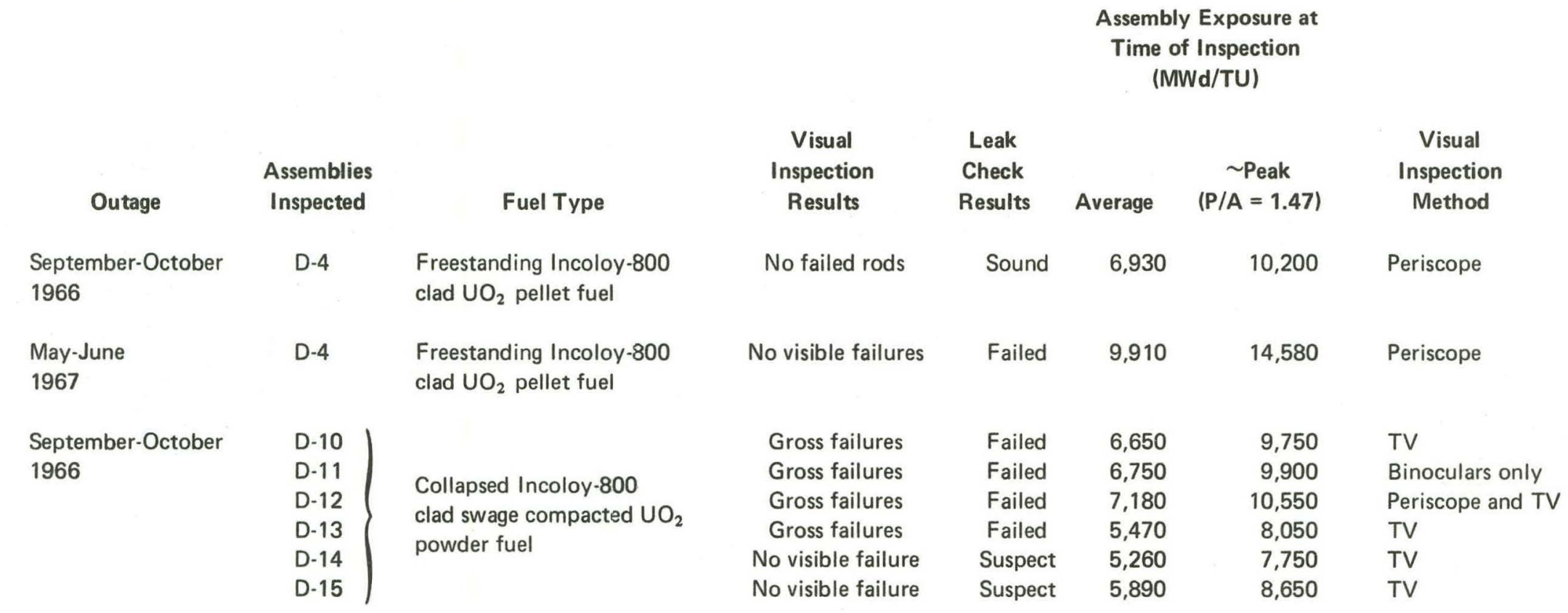




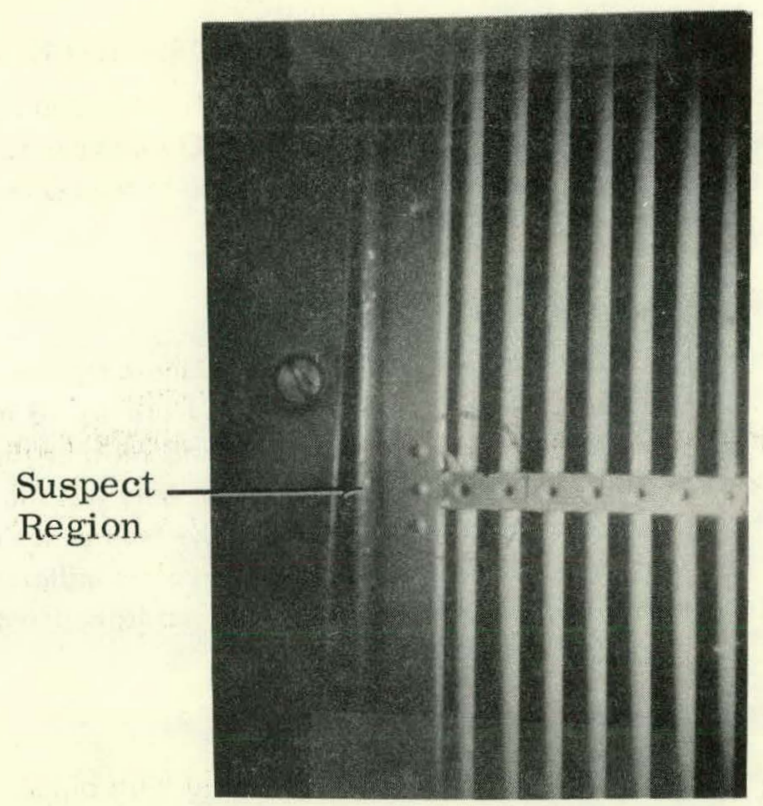

a. Suspect Region on Assembly PO-2

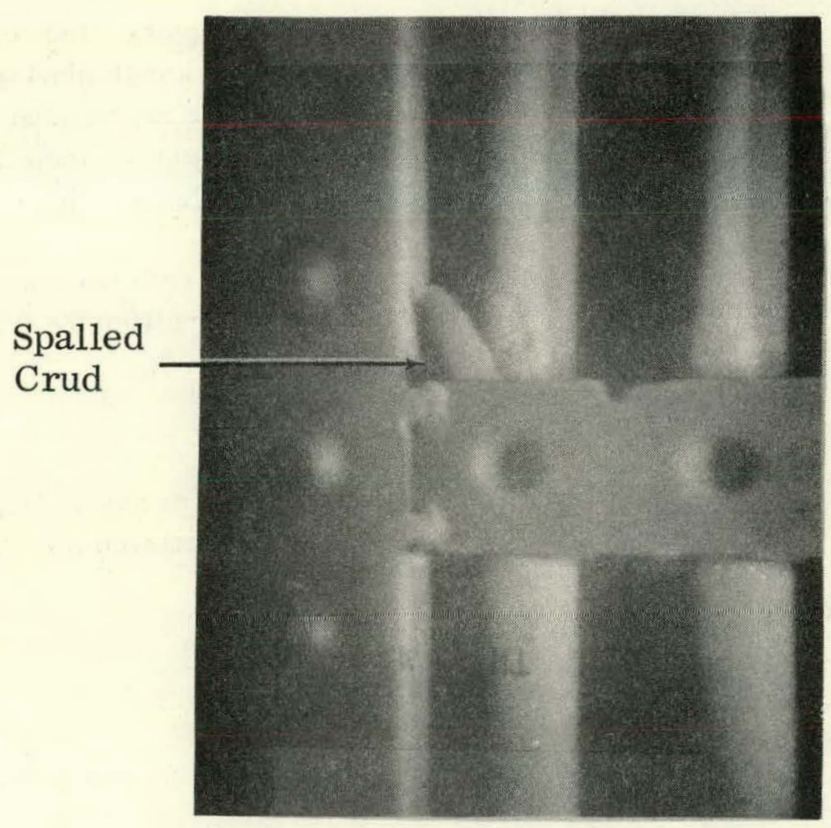

b. Higher Magnification Photo of Suspect Region

Type-304L Stainless Steel Cladding, Collapsed 0.010-inch Wall Swage Compacted $\mathrm{UO}_{2}$ Powder Fuel

Burnup: $7680 \mathrm{MWd} / \mathrm{TU}$ Average

Peak Fuel Rod Surface Heat Flux: $384,000 \mathrm{Btu} / \mathrm{h}-\mathrm{ft}^{2}$

Figure 16. Assembly PO-2, Showing Suspect Failure Region 


\subsubsection{Visual Examination Results for Assemblies D-10, D-11, D-12, D-13, D-14, and D-15 (September-October 1966)}

These assemblies contained fuel rods clad with 0.011 -inch-wall collapsed Incoloy-800 cladding in the cold-worked condition. The fuel rods were fabricated by swage compaction of arc-fused $\mathrm{UO}_{2}$ powder to 90 to $92 \%$ density in thin weld-drawn Incoloy-800 cladding. These fuel rods are similar to those contained in the PO assemblies with Incoloy-800 substituted for the Type-304L stainless steel cladding.

\section{a. Assembly D-10, $6650 \mathrm{MWd} / \mathrm{TU}$ Average Burnup}

The D-10 assembly contained eight peripheral fuel rods with gross failure regions. All the failure regions are located near the spacer band separating the first and second tiers (12 to 18 inches from the top). The failures are longitudinal splits 4 to 12 inches in length in the cladding. Most of the failure regions appear to have lost fuel. Probing of one fuel rod with a tool revealed that not only was the fuel missing in the failure region but also at least 10 to 12 inches of additional fuel above the failure was missing at the time of the inspection. Similar probing of about six additional fuel rod failure regions indicated that very little fuel was missing above the actual failure region. Cladding samples were removed from one of the gross failure regions for further examination.

\section{b. Assembly D-11, $6750 \mathrm{MWd} / \mathrm{TU}$ Average Burnup}

This assembly was not inspected in detail. The assembly was viewed with binoculars and has several gross failure areas.

c. Assembly D-12, $7180 \mathrm{MWd} / \mathrm{TU}$ Average Burnup

This assembly contained five peripheral fuel rods with visible failed regions. Three of the more severe failure regions are located in the second tier from the top. These failures are longitudinal splits in the cladding 7 to 11 inches in length, with most of the fuel missing from the failure region when inspected. No fuel was missing above the failure regions. Figure $17 \mathrm{~A}$ is a photograph of one of these failure regions. A more detailed photograph of this failure region just above a spacer band is shown in Figure $17 \mathrm{~B}$.

In addition to these failures, three fuel rods (one of the above fuel rods has two distinct failure regions) have multiple longitudinal cladding cracks in the third and fourth tiers from the top. These failures are not open, and little or no fuel loss occurred.

\section{d. Assembly D-13, $5470 \mathrm{MWd} / \mathrm{TU}$ Average Burnup}

This fuel assembly contained one fuel rod with a gross failure region. The overall length of the failure region was estimated to be 19 inches. Obvious fuel loss in the failure region occurred prior to inspection.

e. Assembly D-14, $5260 \mathrm{MWd} / \mathrm{TU}$ Average Burnup

No failed fuel rods were observed in this fuel assembly.

f. Assembly D-15, $5890 \mathrm{MWd} / \mathrm{TU}$ Average Burnup

No failed fuel rods were observed in this fuel assembly.

\subsubsection{Post-Irradiation Examination of Failed Incoloy-800 Fuel Rod Cladding}

Two pieces of cladding were removed from a gross fuel rod failure region of a-fuel rod in assembly D-10 and shipped to the hot-cell facility at the Vallecitos Nuclear Center for examination. The objective was to determine the nature of the cladding failures. These failures were the first Incoloy- 800 fuel rod cladding failures observed in a boiling water reactor. The prime interest was to determine whether or not the Incoloy-800 failures resembled the earlier stainless steel failures observed in various boiling water reactors. ${ }^{2}, 24,25$ Later, complete fuel rods and parts of fuel rods from assembly D-12 were shipped to the hot cells.

\section{a. Visual Examination}

Visual examination of the failed Incoloy-800 fuel rods indicated severe failure with many straight-line cracks, shown in Figures 18 and 19. The slight irregularities and branches at the fracture surfaces indicate that they are probably. intergranular. 


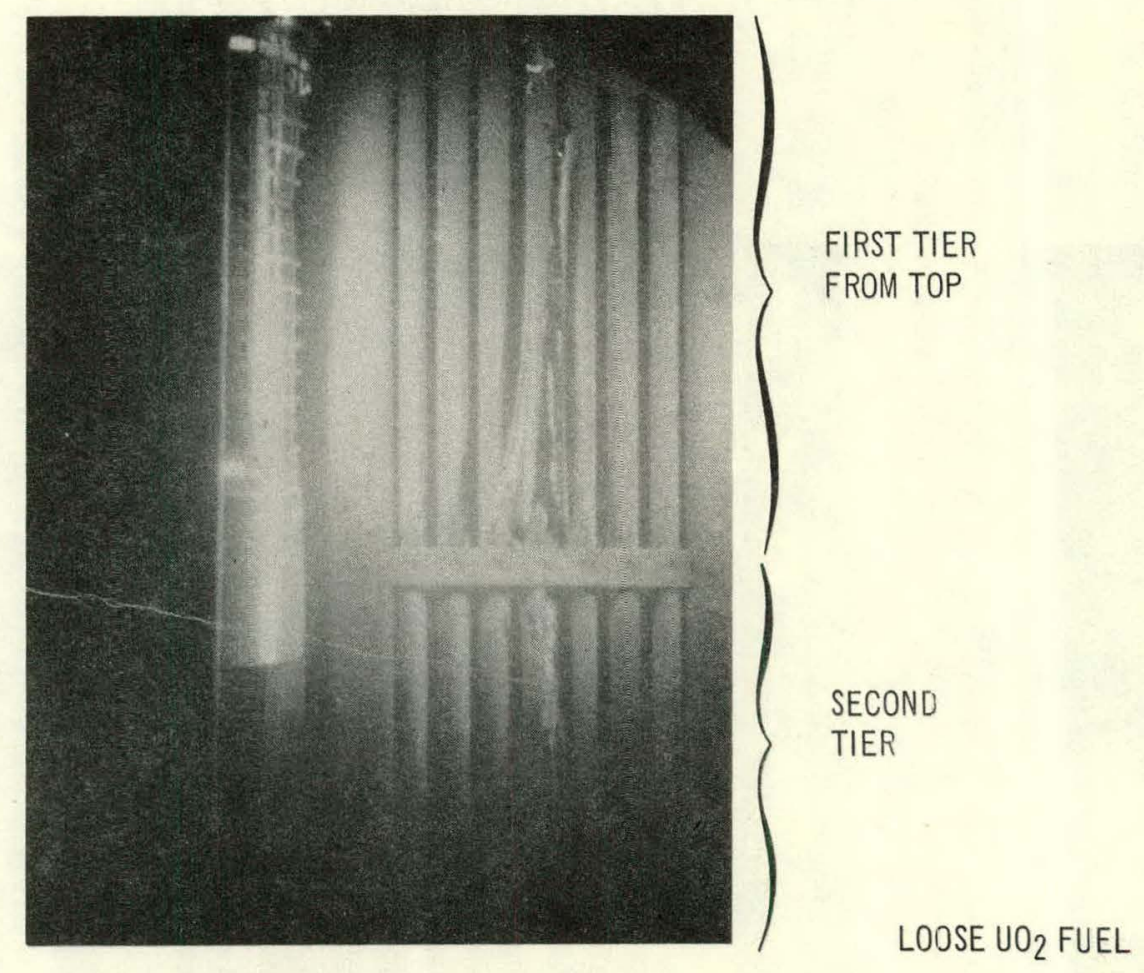

LONG TUDINAL SPLITS IN PERIPHERAL FUEL ROD

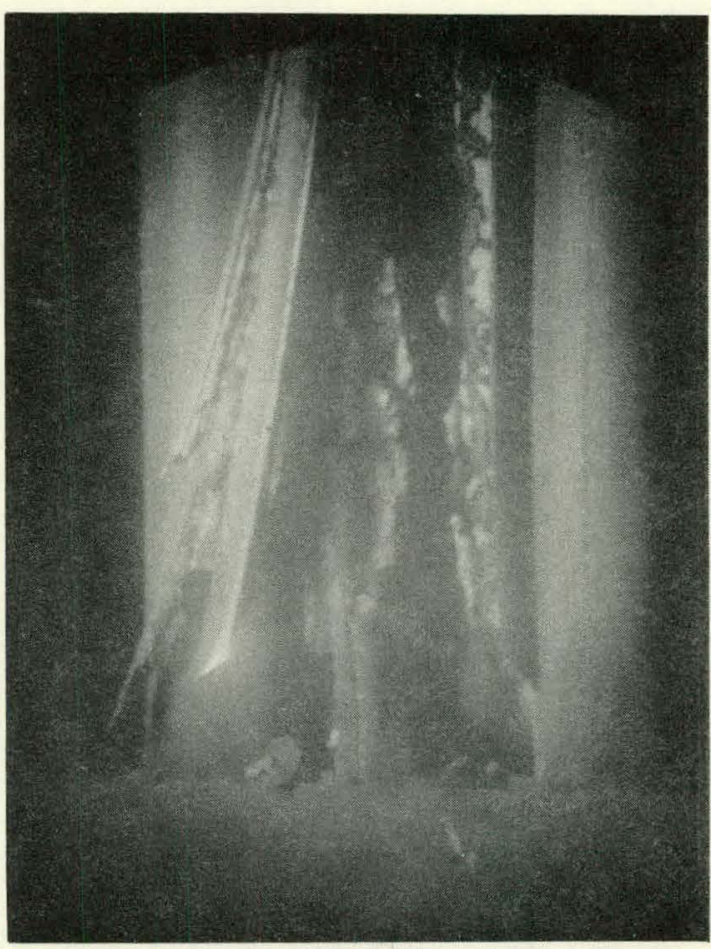

HIGHER MAGNIFICATION PHOTOGRAPH OF GROSS FUEL ROD FAILURE REGION

$1 b$

INCOLOY-300 CLADDING, COLLAPSED 0.011-INCH WALL U0 2 POWDER FUEL SWAGE COMPACTED TO 91 PERCENT DENSE, $7180 \mathrm{MWd} /$ TU AVERAGE ASSEMBLY BURNUP. 


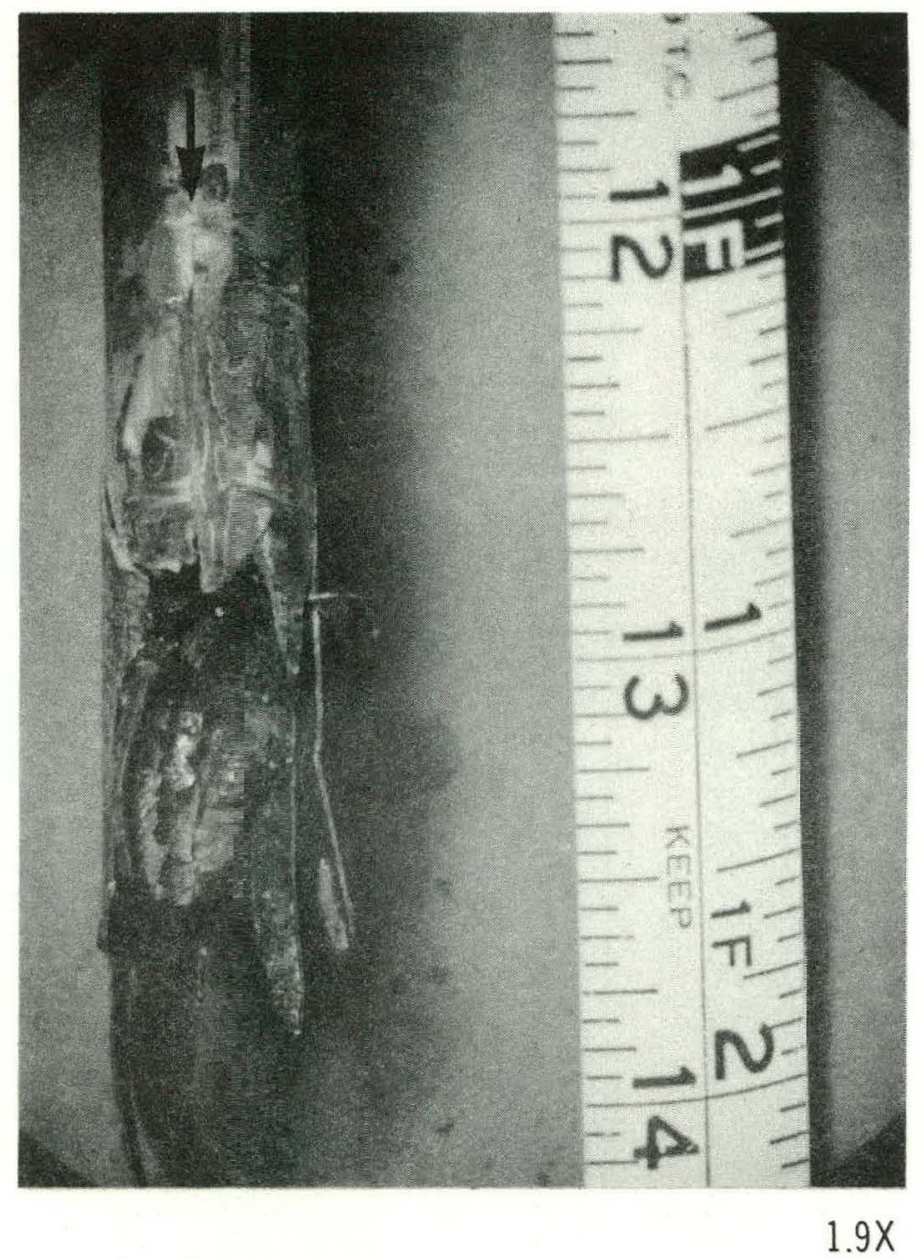

Figure 18.

Fractuie of lincoloy-800 Clad Rod CK-587. A Straight Lin $\equiv$ Crack Extends from the Arrow Downward.

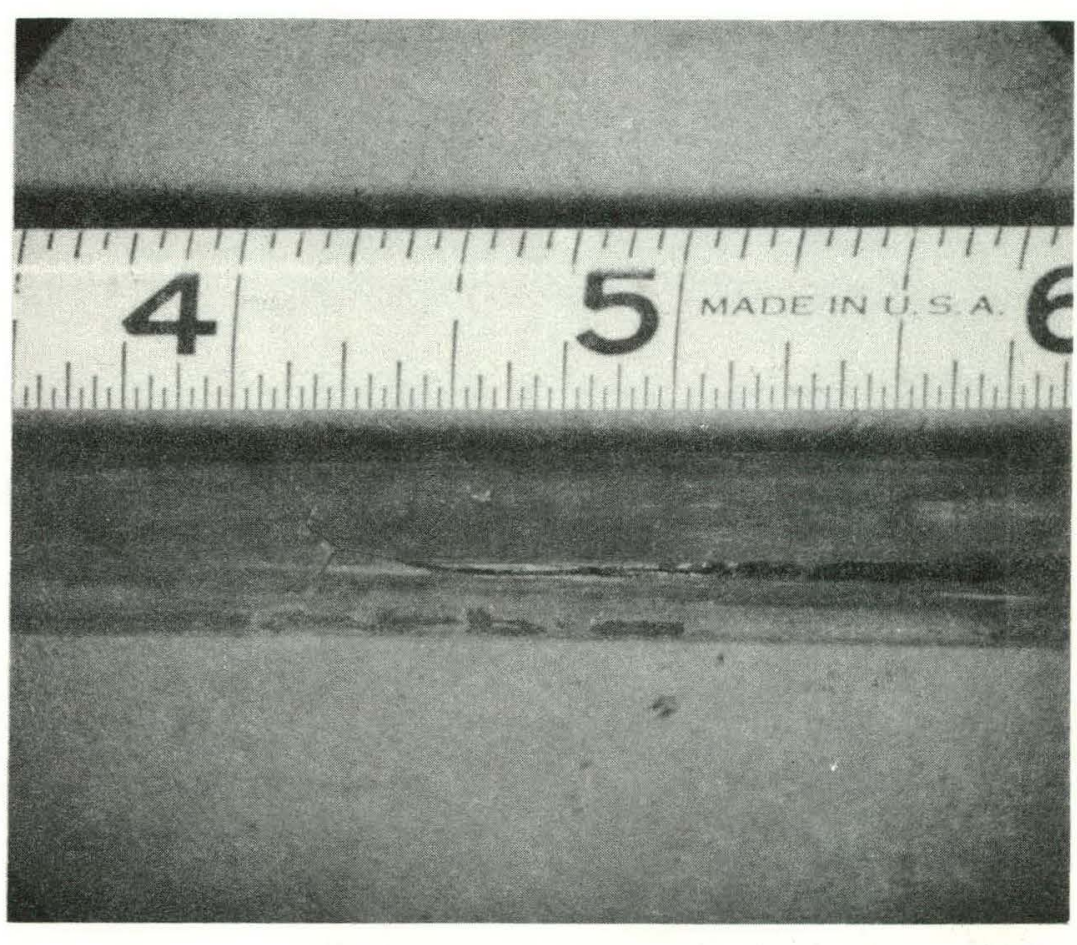


The two bent samples (Figure 20 ) of cladding from assembly D-10 were reddish brown in color, typical of long exposure in a boiling water reactor. The cladding appeared ductile during straightening (longitudinal direction) and handling operations. Both transverse and tangential sections were sheared from each piece and mounted for metallography.

\section{b. Metallographic Examination}

Ten transverse sections and four tangential sections were examined. These sections were taken at random from both pieces of cladding.

Of the ten transverse sections, four contained the seam weld. In all cases, a fracture surface was located within the weld (Figure 21). The only part-through cracks, which penetrated into the cladding from the outside diameter, were also observed in the weld zone (Figure 22). All cracks were intergranular, slightly branched, and contained corrosion products similar to that observed in earlier Type-304 stainless steel failures. $^{22}, 24,25$ The fracture surfaces opposite the weld, in the parent metal, were similar. These fractures were also intergranular (Figure 23).

Two of the four tangential sections contained the seam weld on one side of the section. Here, again, one fracture surface was within the weld zone. The only cracks observed in any of the four tangential sections were in the weld zone (Figure 24).

The following general observations are representative for all sections examined. Minor oxide formation, which measured 1 to 3 microns in thickness, was observed on the outside diameter of the cladding. This amount of corrosion is characteristic for the exposure temperature. Very little deposit was observed. Fuel adhered to the inside diameter of the cladding and appeared to be oxidized. The cladding was not sensitized. Oxidation of the fracture surfaces was observed, indicating the initial failure occurred well before the reactor shutdown. The seam weld zone was not completely homogenized, as evidenced by a cored structure. Little evidence of necking or grain distortion was observed adjacent to fracture surfaces examined, indicating that failures in the weld and parent metal occurred without significant deformation. The intergranular nature of the cracks was similar in appearance to earlier stainless steel cracking. It is, therefore, reasonable to associate environmental factors such as localized stress-assisted corrosion with the observed cladding failures. The fact that the weld zone metal was also cracked suggests that the weld zone metal could be an important factor in the time to initial failure. The cold-worked condition of the initial cladding would severely lower ductility at operating temperatures and also add high residual stress which could increase susceptibility to environmental effects.

The following are conclusions based on the examinations:

(1) The longitudinal fractures in the two pieces of Incoloy- 800 cladding examined were in both parent metal and weld metal. All fractures were intergranular.

(2) The only cracks observed which had partial cladding penetration were in the weld zone itself. These cracks were also intergranular, and started from the outside of the cladding.

(3) Very little, if any, necking or grain distortion was observed, indicating that both weld and parent metal failures occurred without significant deformation.

(4) Only minor general corrosion and crud deposits were observed, indicating normal cladding temperatures. The lack of carbide precipitation alsn suggests low temperature opcration.

(5) The observed fractures appear to be similar to Type-304 stainless steel failures which are associated with environmental factors such as stress-assisted localized corrosion. 


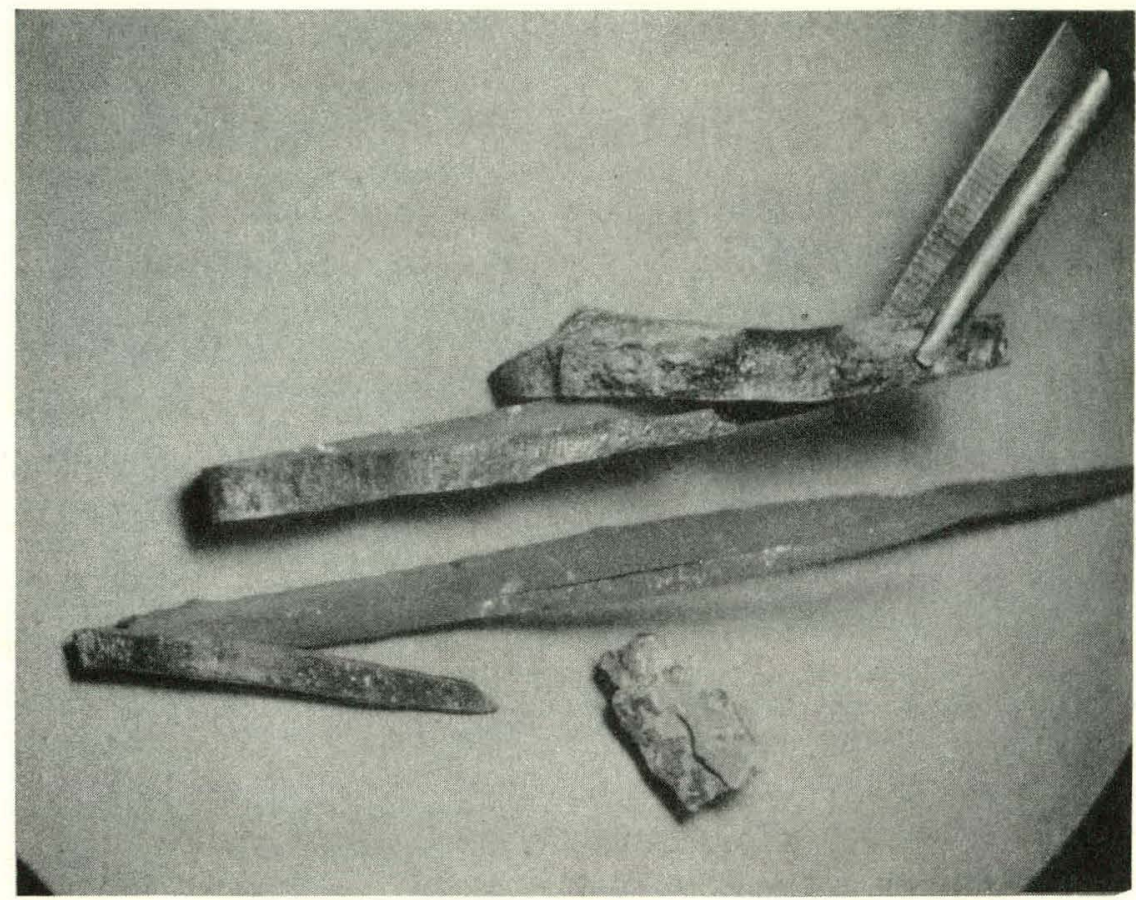

INCOLOY-800 CLADDING, COLLAPSED 0.011-INCH WALL, U02 POWDER FUEL SWAGE COMPACTED TO 91 PERCENT DENSE, $6650 \mathrm{MWd} /$ TU AVERAGE ASSEMBLY BURNUP.

Figure 20. Photomacrograph of Incoloy-800 Cladding Samples from Assembly D-10

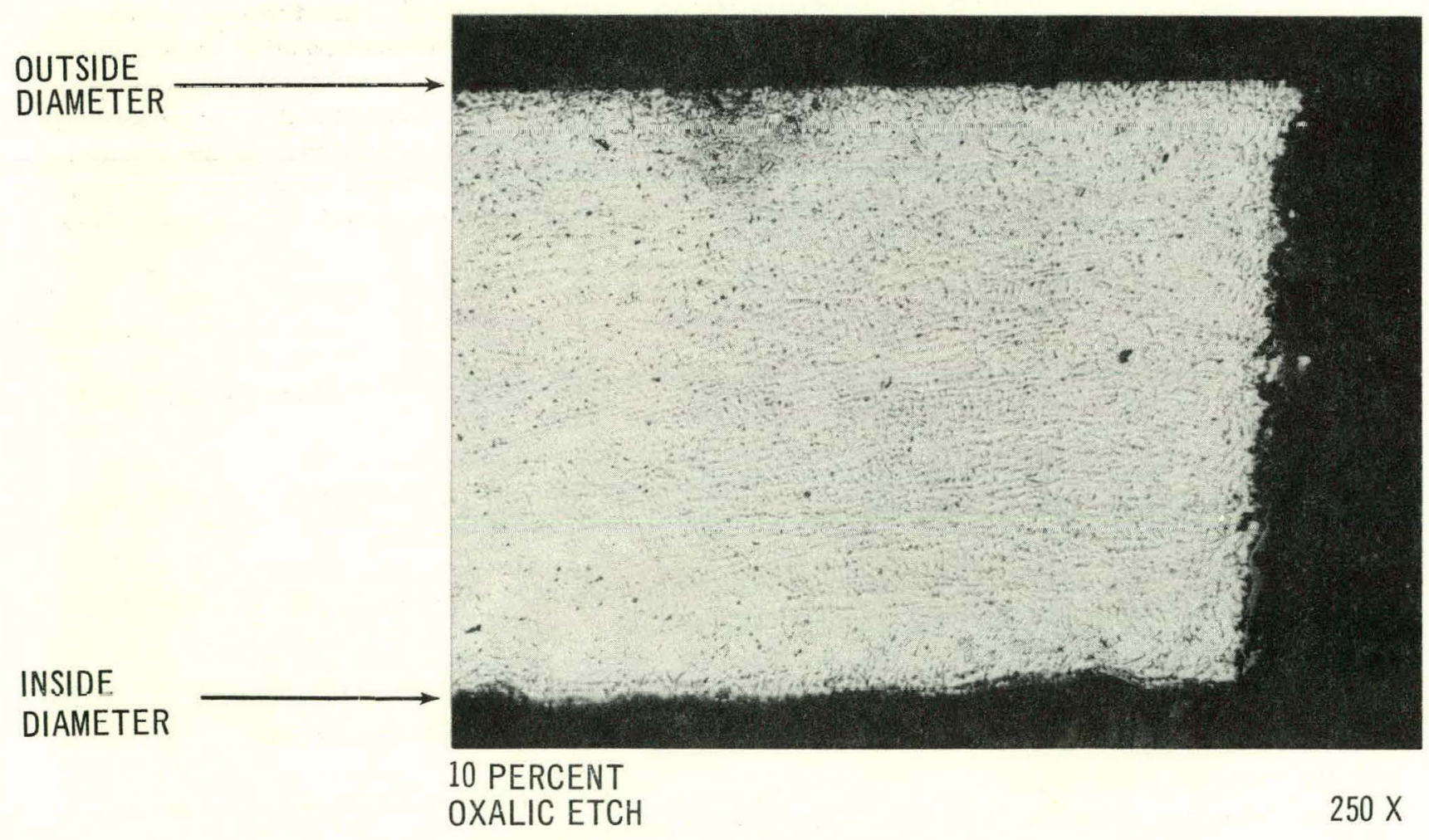

Figure 21. Photomicrograph of Fracture Region in Seam Weld, Incoloy-800 Cladding, Transverse Section 


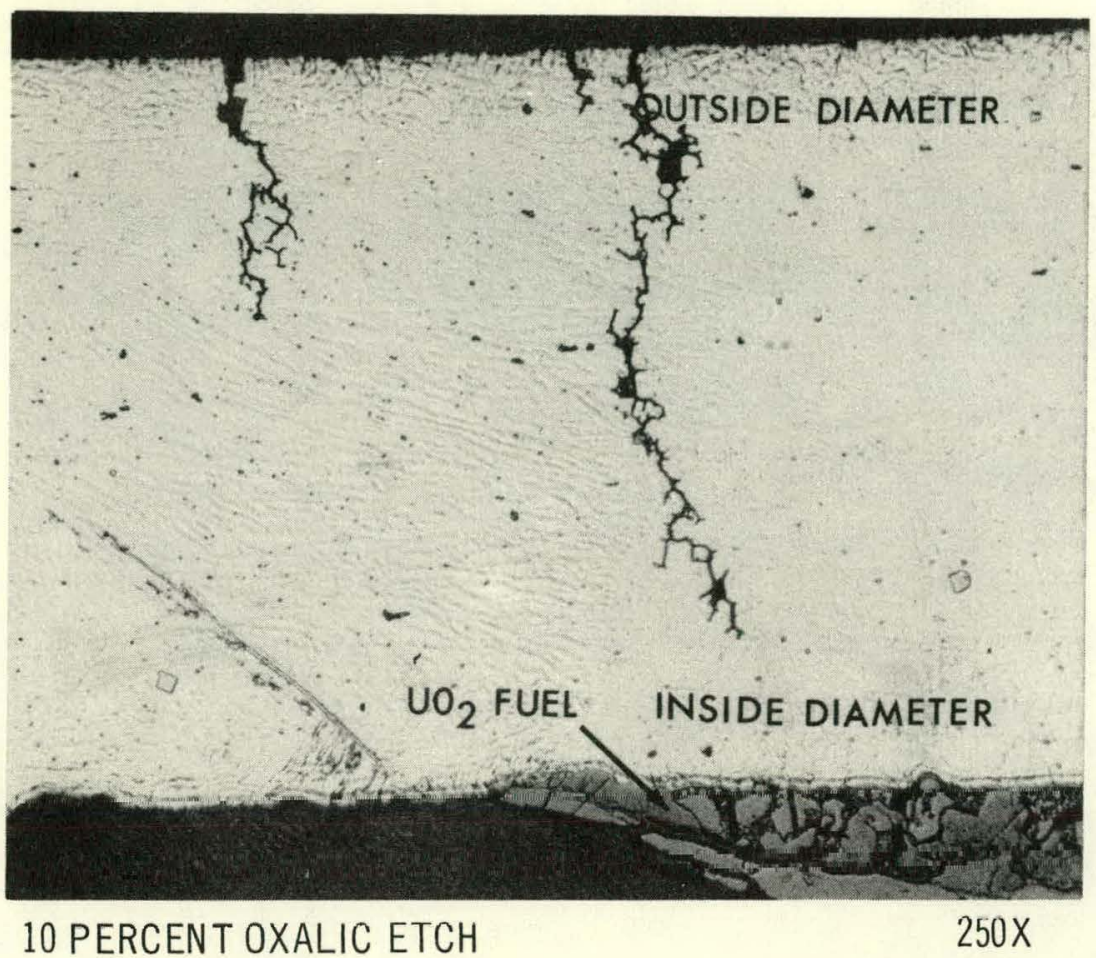

Figure 22. Incoloy-800 Cladding, Intergranular Cracking in Seam Weld Starting on Outside Diameter

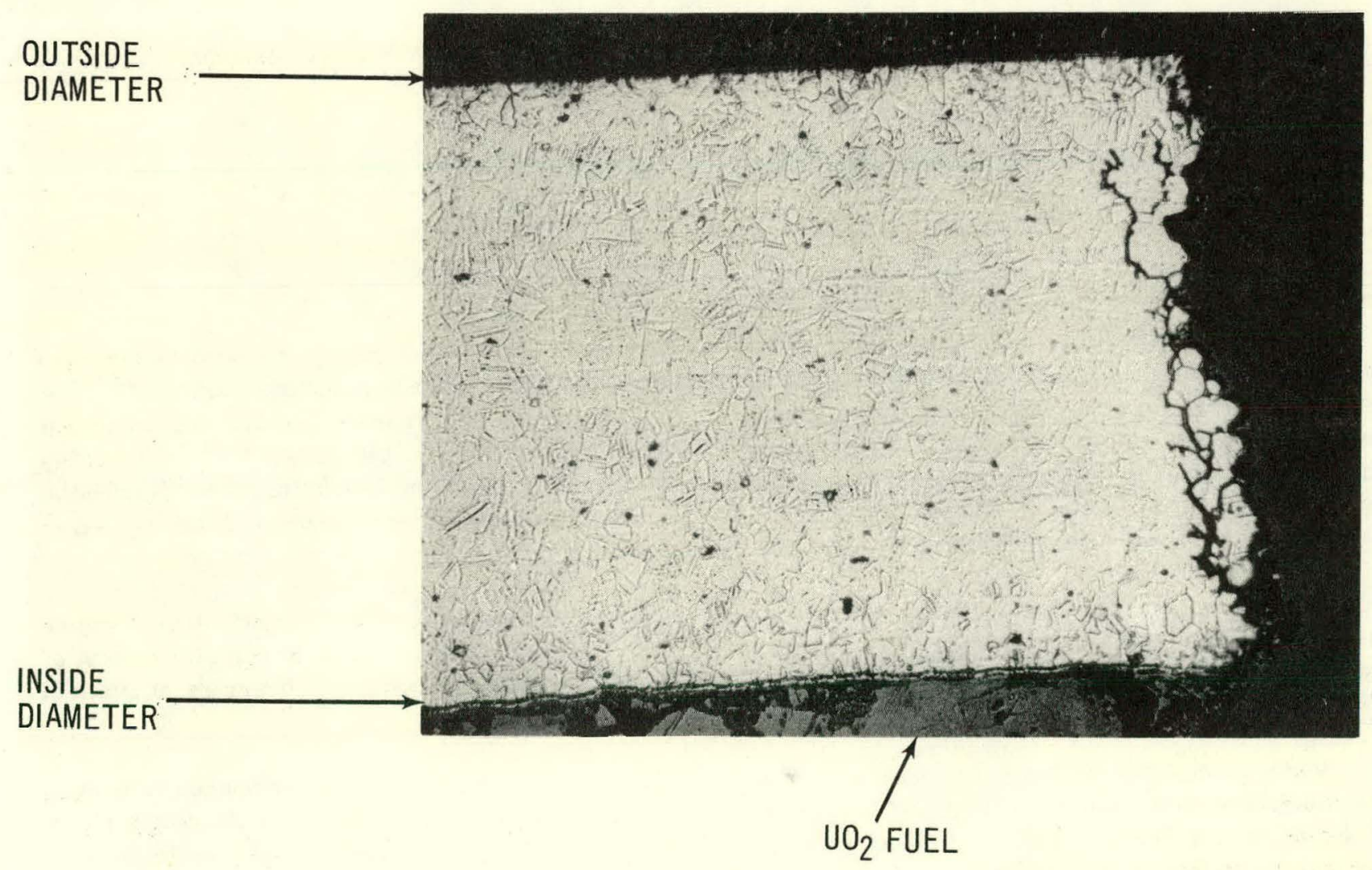

Figure 23. Photomicrograph of Intergranular Fracture Surface in Incoloy-800 Cladding Away from Weld Seam, Transverse Section 


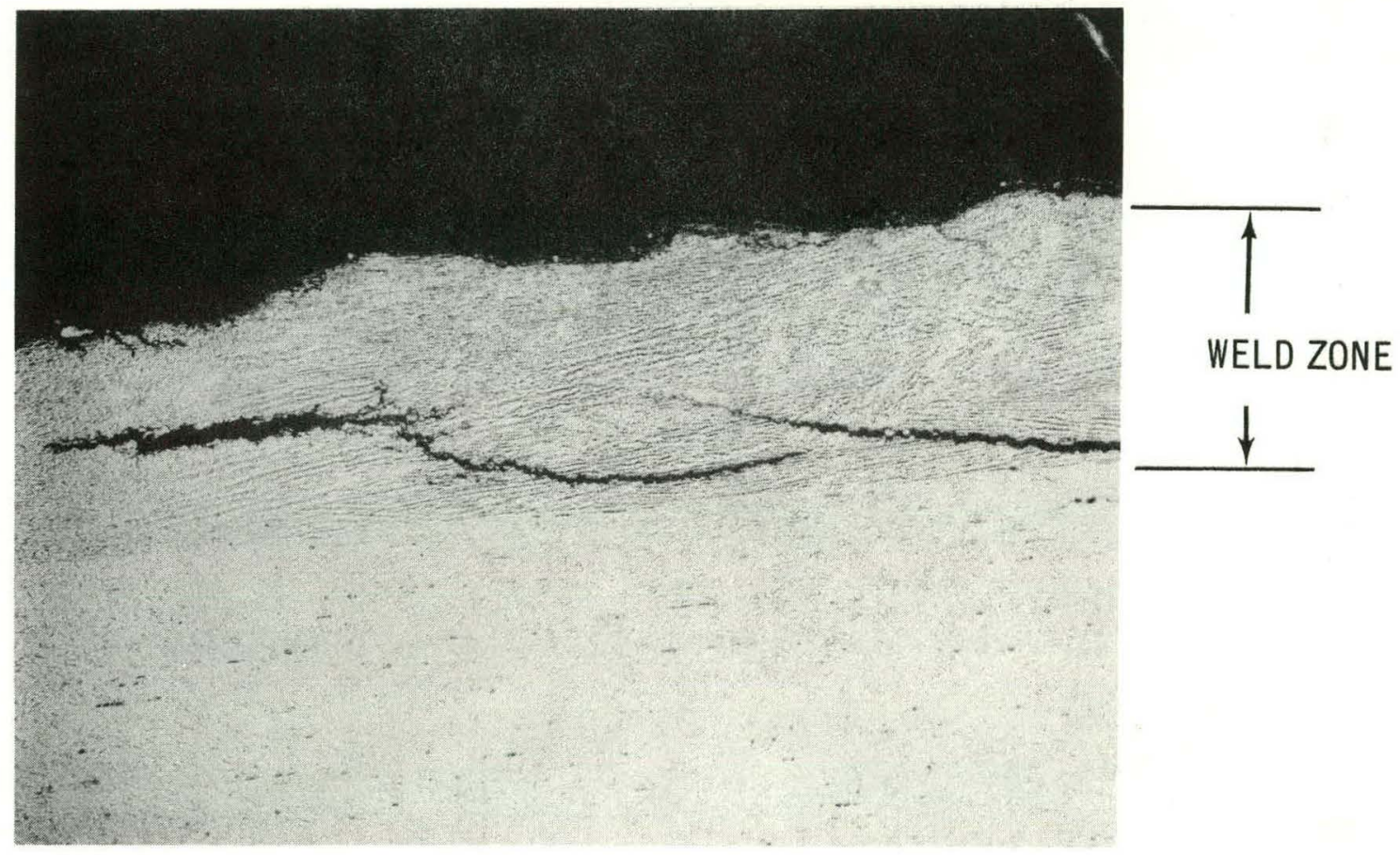

10 PERCENT

OXALIC ETCH

Figure 24. Photomicrograph of Intergranular Cracks in Seam Weld, Incoloy-800 Cladding,

Tangential Section

\subsubsection{Intergranular Corrosion Susceptibility Tests on Incoloy-800}

The intergranular failure mode of Incoloy-800 fuel rod cladding in the Big Rock Point reactor may be similar to the Type-304 stainless steel failures which have been attributed to stress-assisted intergranular corrosion. ${ }^{22,24,25} \mathrm{~A}$ test to determine the relative susceptibility of commercial Incoloy-800 to selective grain boundry dissolution was performed. The test, utilized as part of a program to investigate the cause of stainless steel failures, ${ }^{26,27,28}$ involves exposure of samples in boiling nitric acid solutions containing high valence chromium ions $\left(\mathrm{HNO}_{3}+\mathrm{Cr}^{+6}\right)$ added as potassium dichromate. This test does not simulate a boiling water reactor environment; however, it can be used to determine relative susceptibility of materials to corrosion by grain boundry dissolution.

Previous work on stainless steels has shown that intergranular corrosion susceptibility in $\mathrm{HNO}_{3}+\mathrm{Cr}^{+6}$ can be attributed to solute segregation of impurities, silicon and phosphorus, to the grain boundaries. Stainless steel alloys of high purity or commercial-purity alloys not containing silicon and phosphorus were immune to intergranular attack in $\mathrm{HNO}_{3}+\mathrm{Cr}^{+6} \cdot 27,28$

Various samples were obtained for testing from thin-walled Incoloy-800 tubing used to fabricate one of the fuel rods which failed in the reactor in fuel assembly D-12; from Incoloy-800 tubing used to fabricate free-standing fuel rods for assemblies D-4, D-5, and D-6; and from a high-purity $20 \% \mathrm{Cr}-30 \%$ Ni-balance Fe experimental alloy which could be considered to be high-purity (not commercial) I ncoloy- 800 . 
The Incoloy- 800 aloys were exposed to a boiling $5 \mathrm{~N} \mathrm{HNO}_{3}-0.46 \mathrm{~N} \mathrm{Cr}^{+6}$ solution for 14 hours to evaluate their relative resistance to intergranular corrosion.

The specimens were representative of:

a. Commercial-purity Incoloy-800 tubing used to fabricate collapsed cladding, 0.011-inch-wall swage-compacted fuel rods for assemblies D-10, D-11, D-12, D-13, D-14, and D-15.

b. Commercial-purity Incoloy-800 tubing used to fabricate free-standing, 0.019-inch-wall fuel rods for assemblies D-4, D-5, and D-6.

c. Experimental high-purity alloy containing $50 \mathrm{ppm}$ silicon and $100 \mathrm{ppm}$ phosphorus.

All specimens were tested in tubing form. The results of these tests are shown in Figure 25. Both commercial-purity alloys corroded at a high rate $\left(5.5\right.$ to $\left.5.7 \mathrm{mg} / \mathrm{cm}^{2}-\mathrm{h}\right)$ while the high-purity alloy corroded at a low rate $\left(0.75 \mathrm{mg} / \mathrm{cm}^{2}-\mathrm{h}\right)$. This corrosion behavior indicated that the high weight losses by the commercial-purity alloys resulted from intergranular penetration and grain dropping. The low weight loss rate of the high-purity alloy indicated that the alloy corroded by general dissolution.

Metallographic examination confirmed the different modes of weight loss. It was apparent that both commercial-purity alloys corroded intergranularly, while the high-purity alloy corroded by uniform surface dissolution only.

The present results (that high-purity alloys are immune to intergranular attack while commercial-purity alloys are not) are in complete agreement with the studies performed on other stainless steel compositions. ${ }^{27,}{ }^{28}$

\subsection{ZIRCALOY-2 CLAD-UO ${ }_{2}$ POWDER FUEL RODS (FAILED FUEL ASSEMBLIES D-18, -19, AND -20)}

During the September-October 1966 inspection, leak checks of assemblies D-18, -19, and -20 indicated that all three of these assemblies contained failed fuel rods. Examination of these assemblies revealed no visible fuel rod failures.

Work was initiated in January 1967 to prepare several developmental fuel rods for shipment to the General Electric hot cells at the Vallecitos Nuclear Laboratory. An initial step was to take fission product activity measurements of small groups of fuel rods from assembly D-19 (Zircaloy-2 clad, powder fuel) in an attempt to isolate individual failed fuel rods. These attempts were unsuccessful. However, bubbles were observed coming from one rod. A small amount of fission product $\mathrm{Kr}-85$ was detected in these bubbles, so this rod (CR-163) was included in the shipment. Other preparatory work included underwater puncturing of the fuel rods and collection of the fission gas in tanks, and finally shearing the rods in half so they would fit in a cask small enough to be handled at the hot cells. The rods returned are listed in Section 3, Table 3.

During the puncturing operation, fission gases were collected in vacuum tanks. Radiation readings from these tanks were 2 to $8 \mathrm{mR} / \mathrm{h}$. The expected radiation reading is about $200 \mathrm{mR} / \mathrm{h}$ for a tank containing $1 \%$ of the $\mathrm{Kr}-85$ generated in one of the above fuel rods. Thus, measured dose rates indicate that only a small fraction of a percent of the generated fission gases was released and/or collected.

Gas from rod CR-125 was analyzed by gas chromatography with the results listed as follows:

\begin{tabular}{lcrrrrr}
\multicolumn{1}{c}{ Gas } & $\mathrm{O}_{2}$ & $\mathrm{~N}_{2}$ & $\mathrm{Xe}$ & $\mathrm{Kr}$ & $\mathbf{H}_{2}$ & \multicolumn{1}{c}{$\mathbf{H e}$} \\
Volume, \% & 19.0 & 79.9 & 0.22 & Trace & 0.12 & 0.33 \\
Precision & \pm 1.0 & \pm 2.0 & \pm 0.02 & & \pm 0.02 & \pm 0.01
\end{tabular}




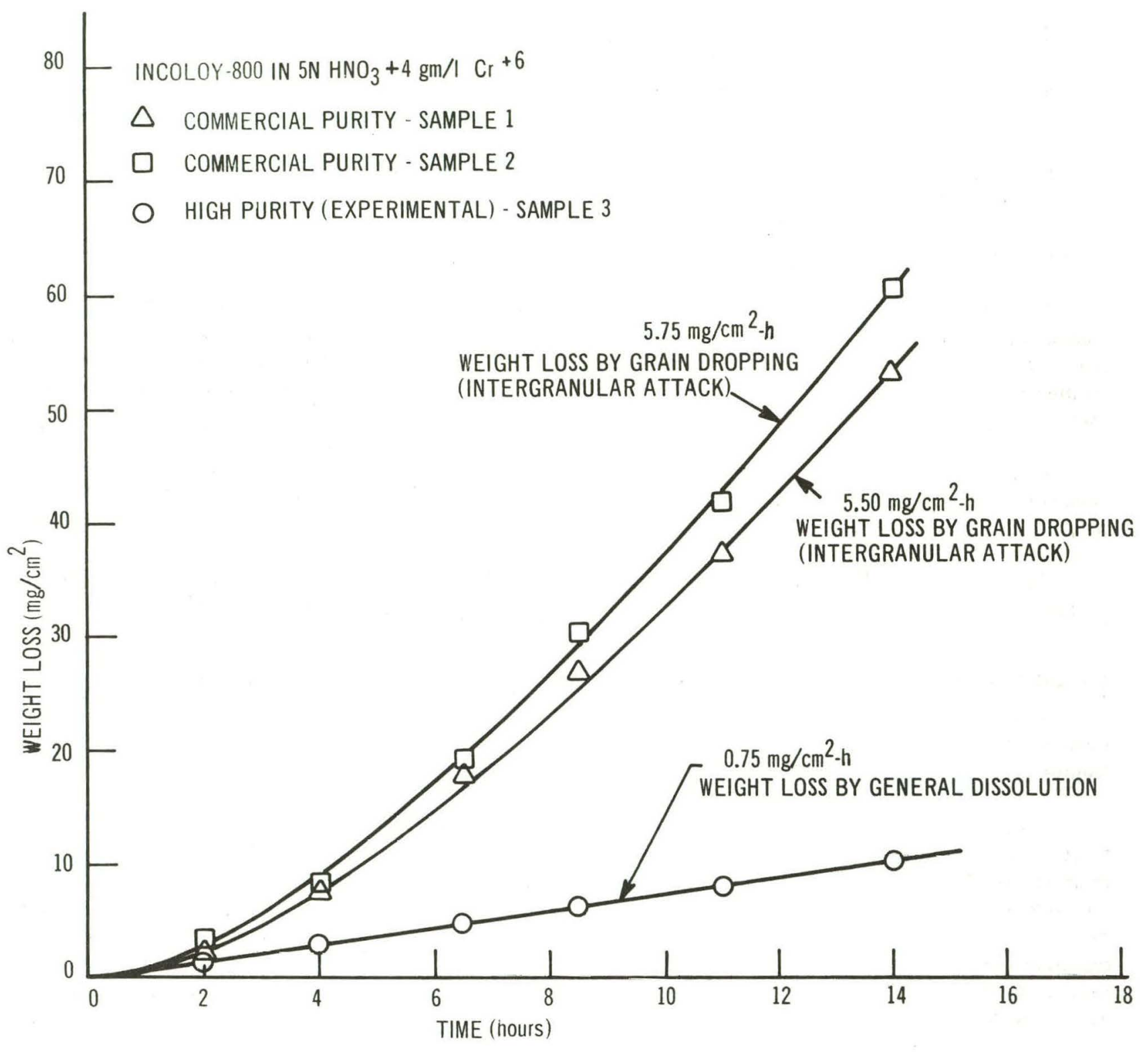

Figure 25. Corrosion Behavior of Various Incoloy-800 Samples in $\mathrm{HNO}_{3}-\mathrm{Cr}^{+6}$ Solution (Weight Loss versus Time) 
Air inleakage is obvious in this analysis, but the time and location of its entry is uncertain. The gas collection tanks were helium leak-checked during construction but a small leak may have developed, or a foreign particle may have lodged on the valve seat preventing full closing. An air leak of $10^{-6} \mathrm{~cm}^{3} / \mathrm{sec}$ would account for the quantity of oxygen and nitrogen observed. Any out-diffusion from the tank would preferentially reduce the quantities of the lighter gases, making the above proportions incorrect. If, however, the above ratio of hydrogen to helium represents what was in the rod, it is evidence that CR-125 was unfailed.*

\subsubsection{Nondestructive Examination}

The nondestructive examination consisted of visual examination of all rods, and gamma scanning, dimensional profiling, leak testing, and neutron radiographing of selected rods.

\subsubsection{Visual Examination}

The cladding surfaces had an irregular brownish-red to black appearance. The lower end plug was missing from Zircaloy-2 clad powder fuel rod CR-163. This is shown in Figure 26A. It was estimated that 0.1 inch of the fuel column was missing, in addition to the end plug. Part of the lower end plug was subsequently found wedged in the lower spacer band of assembly D-19, which contained rod CR-163 (Figure 26B). Apparently the cladding cracks were so fine or else covered with deposits that they could not be seen when this assembly was originally visually examined after removal from the reactor. An intact end plug is shown in Figure 26C for comparison.

The powder fuel was well packed and remained in place both at the sheared locations and at the missing end plug. Little, if any, fuel fell out of the cladding tubes during shipping and handling.

\subsubsection{Leak Testing}

All four $\mathrm{CR}$ rods (Zircaloy-2 clad $\mathrm{UO}_{2}$ powder fuel) were leak tested to determine whether any of the other powder fuel rods besides CR-163 were failed, and to pinpoint the location of the penetration near the upper end plug of CR-163 ${ }^{\dagger}$. The deliberate puncture holes and lower end of CR-163 were sealed with epoxy resin. The rods were then pressurized with 20-psi argon and immersed in water. No bubbles were emitted from any of the rods, except for the top end of CR-163. Figure 27 shows the CR-163 circumferential crack located by the leak testing.

\subsubsection{Neutron Radiography}

The ends of rod CR-163 and rod CR-125 were neutron radiographed to compare the appearances of end plug regions for a failed rod with an intact rod. The neutrograph is shown in Figure 28. Darker areas in the positive prints correspond to white areas on the negative which, in turn, are due to the attenuation and scattering of neutrons. Thus, the fuel and hydrogenous material appear dark and the cladding is light in the figure.

The curved upper surface of the CR-125 fuel column was caused by the cupped surface of the separator disc. The dark spots on the cladding surface are epoxy resin used to seal the fission gas collection puncture hole. The dark particles between the fuel column surface and end plug could be either fuel particles or pieces of the separator disc. The CR-125 end plug appears darker than the CR-163 end plug. It is uncertain whether this is a real indication of relative hydride content or merely from a residual epoxy (hydrogenous) film on CR-125.

CR-163 does not have a well-defined upper fuel column surface, and the fuel has moved up aqainst the end plug, indicating failure of the separator disc. The dark spot in the cladding is zirconium hydride at the crack (see subsection 4.3.2).

* Previous results at the Vallecitos Nuclear Center have shown that failed fuel rods typically contained much more hydrogen than helium, probably a result of water entry and chemical interactions which release hydrogen.

I Bubbles were observed coming from the upper part of this rod during preparation for shipping. 


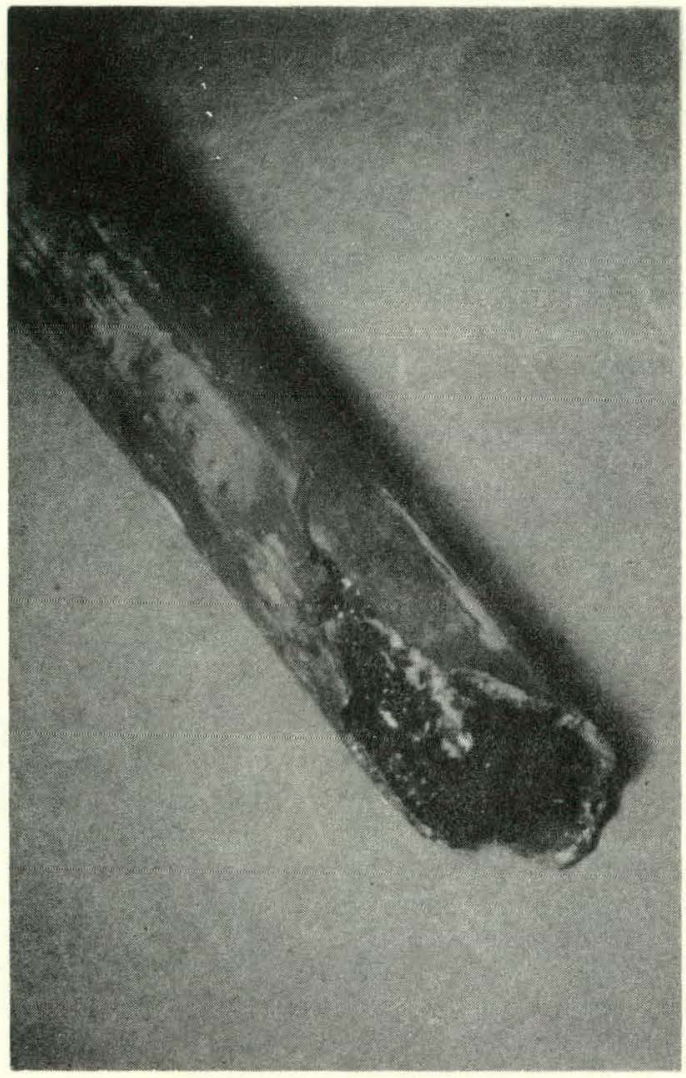

$1.9 \times$

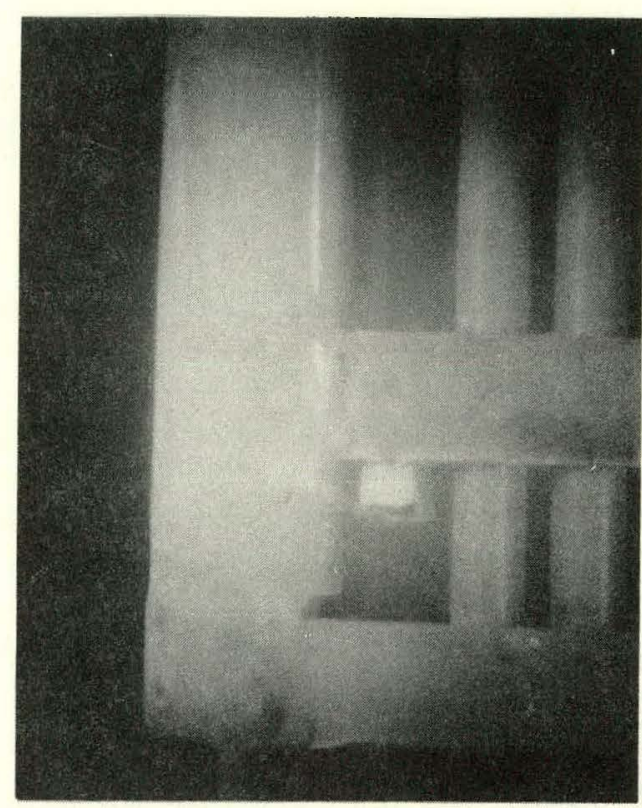

$0.7 X$

Figure 26B. Part of Lower End of Rod CR-163 Wedged at the Lower Spacer Band of $\Delta$ ssemhly $n-19$

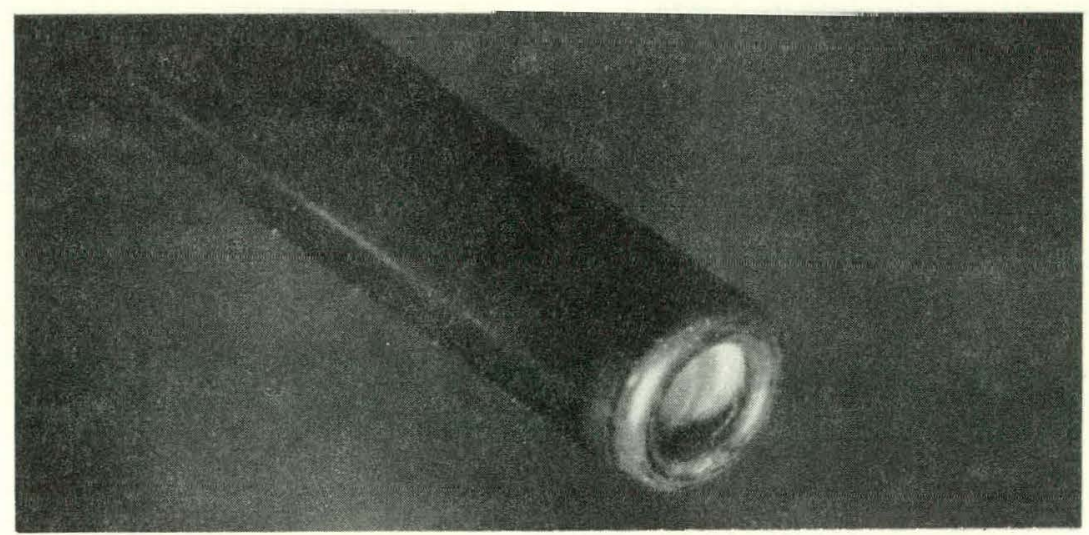

Figure 26C. An Intact Lower End Plug of a Zircaloy-2 Clad Powder Fuel Rod 


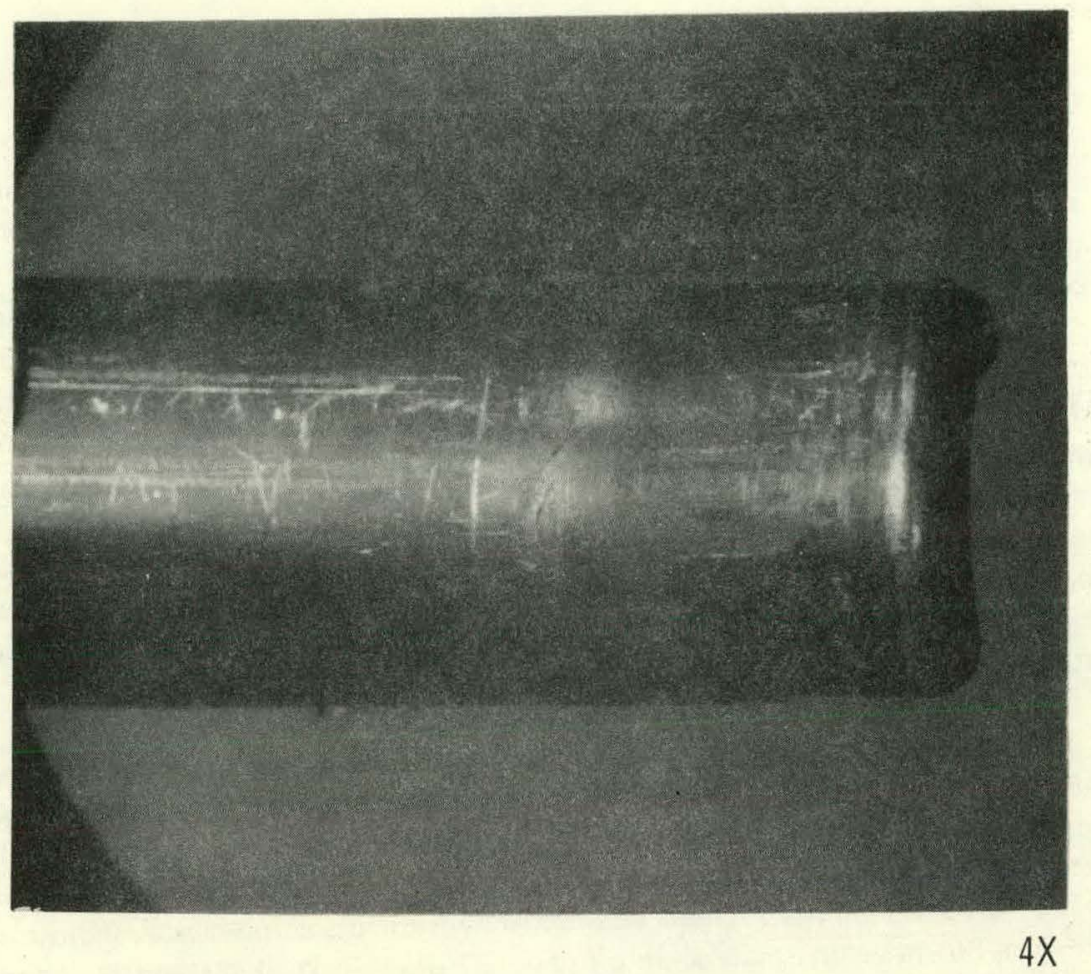

Figure 27. Circumferential Crack at Top of Zircaloy-2 Clad Rod CR-163 After Initial Cutting of Metallographic Section
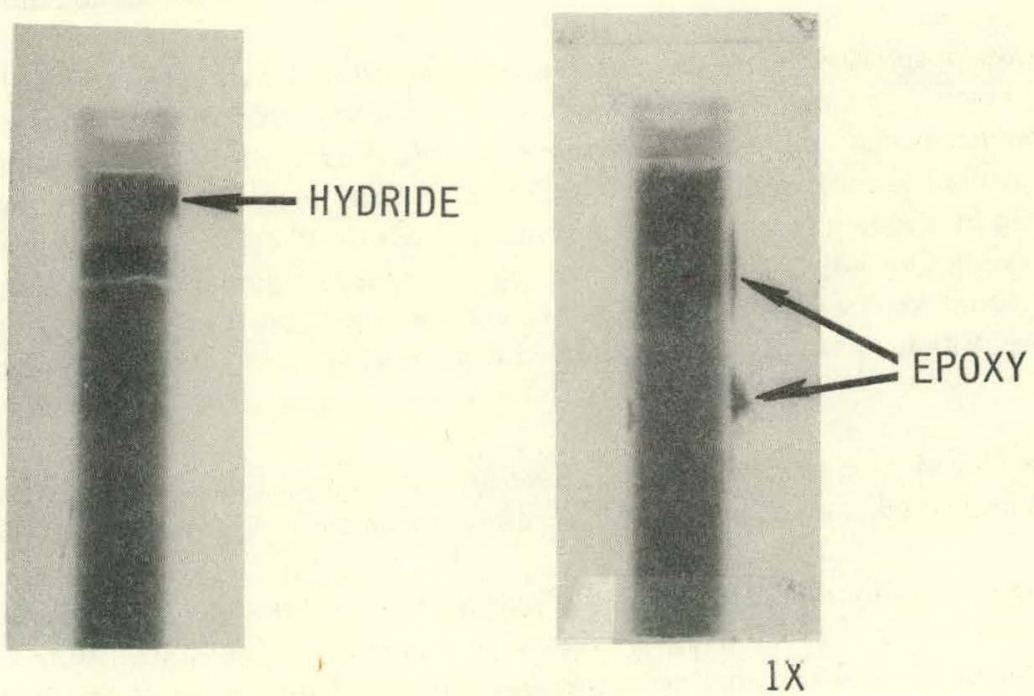

Figure 28. Comparison Neutron Radingraphs of Zircaloy-2 Clad Fuel Rods CR-163 and CR-125, Top End 


\subsubsection{Metallography}

\subsubsection{Zircaloy-2 Clad, $\mathrm{UO}_{2}$ Powder Fuel Rods}

Longitudinal sections were made of each end of both CR-163 (failed) and CR-125 (sound). Several different planes of the upper end of CR-163 were examined. Much of the fuel and the separator disc were lost during preparation of the sample. The circumferential crack was present in each of the planes. Figures 29A, 29B, and 30 show the crack and the adjacent massive cladding hydriding. The cladding has locally thickened about $10 \%$ because of the increased volume of the hydride and has also bulged outward. It was not clear whether the bulge was totally the result of the hydriding or was also influenced by radial loads from the separator disc which was at or near the crack. Most partial cracks initiated at the inner surface of the cladding. Some of the cracks had been exposed long enough to have the fracture surfaces oxidized. The cladding hydride content was significantly lower at regions away from the cracks.

The metallographic section of the CR-163 lower end is shown in Figures 31 and 32 . As at the upper end, there is massive local cladding hydriding and thickening, with the partial cracks occurring on the inner surface. Approximately 1 inch above the cracks, the hydride level diminishes to normal. The fuel particles appear to have a second phase at their surfaces-probably higher oxides.

The upper end plug section of rod CR-125 is shown in Figures 33, 34, and 35. The cladding and end plug have a slightly higher hydride content than regions away from the upper end, although they are still intact. The separator disc has deteriorated, apparently having become very brittle. The layered structure of the cracks is similar to Figure $29 \mathrm{~A}$ and to Figure 4.3 of Reference 29 , but analyses have not been made of the disc to verify the presence of hydride. The lower end section of CR-125 appeared normal. There was little hydriding and no visual indication of overheating or mechanical damage. Figure 36 shows the macrograph. Figure 37 shows part of the end plug and the typical cladding condition in the area.

\subsubsection{Analysis of Failure of Zircaloy-2 $\mathrm{Clad} \mathrm{UO}_{2}$ Powder Fuel Rods}

The examination revealed that one out of four rods (CR-163) returned had failed, with a cladding crack near the upper end plug and the lower end plug broken off. The primary, or first, cause of failure cannot be unequivocally determined. There is some evidence to support internal hydriding from hydrogenous impurities in the rod as the failure mechanism. The final rod closure was effected by evacuating the rod to less than $100, \mu$ at room temperature and backfilling with helium. Some high-temperature outgassing was applied to the fine particle $\mathrm{UO}_{2}$ but, in retrospect, the total control over powder gas content was not sufficient to positively preclude hydrogenous contamination.

For this mechanism, the postulated failure sequence would be as follows: Hydrogen released from the undetected contaminant preferentially reacted at the cladding disc region and massively hydrided both the cladding and disc. The reaction was abetted by the mechanical contact between the cladding and disc, which served to rupture their protective oxide films. The volume increase of the hydrided disc and/or fuel radial expansion stressed the hydride embrittled cladding eventually resulting in cladding cracking. Fuel expansion loads on the disc ultimately fractured the disc and allowed the fuel to redistribute upward, as seen in the neutron radiograph, Figure 28. Water entering the rod at this upper crack would then account for the subsequent hydriding and fracture of the lower end plug and its fracture as the rod was removed. Lower end plug failure has been observed previously as a secondary failure after the rod failed elsewhere. $^{30}$

Alternatively, the lower end plug of CR-163 might have been the actual first point of initial water entry. This could have occurred from a poor weld, a crack induced by vibratory compaction, or from internal hydriding.

On balance, the hydrogenous impurity explanation is somewhat favored because of the CR-125 rod evidence (i.e., a disintegrated brittle disc in a sound rod) and because of the high frequency of failure (i.e., all three assemblies of this type indicated fission gas release. ${ }^{21}$ This explanation is also consistent with the failure diagnosis at other laboratories where similar failure characteristics-local blisters from massive hydriding-were encountered with Zircaloy-2 clad fuel. 29,31 


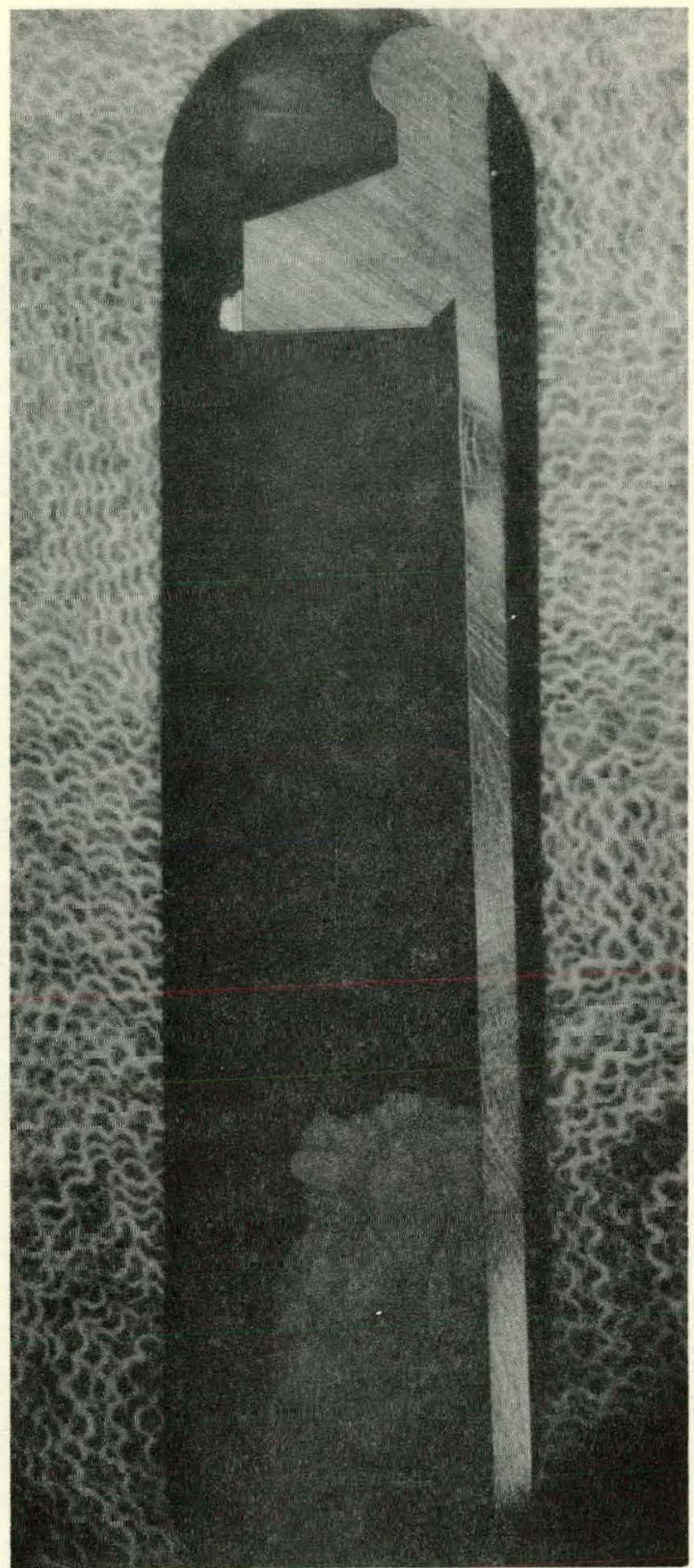

AS POLISHED

Figure 29A. First Metallographic Plane of CR-163, Upper Failure

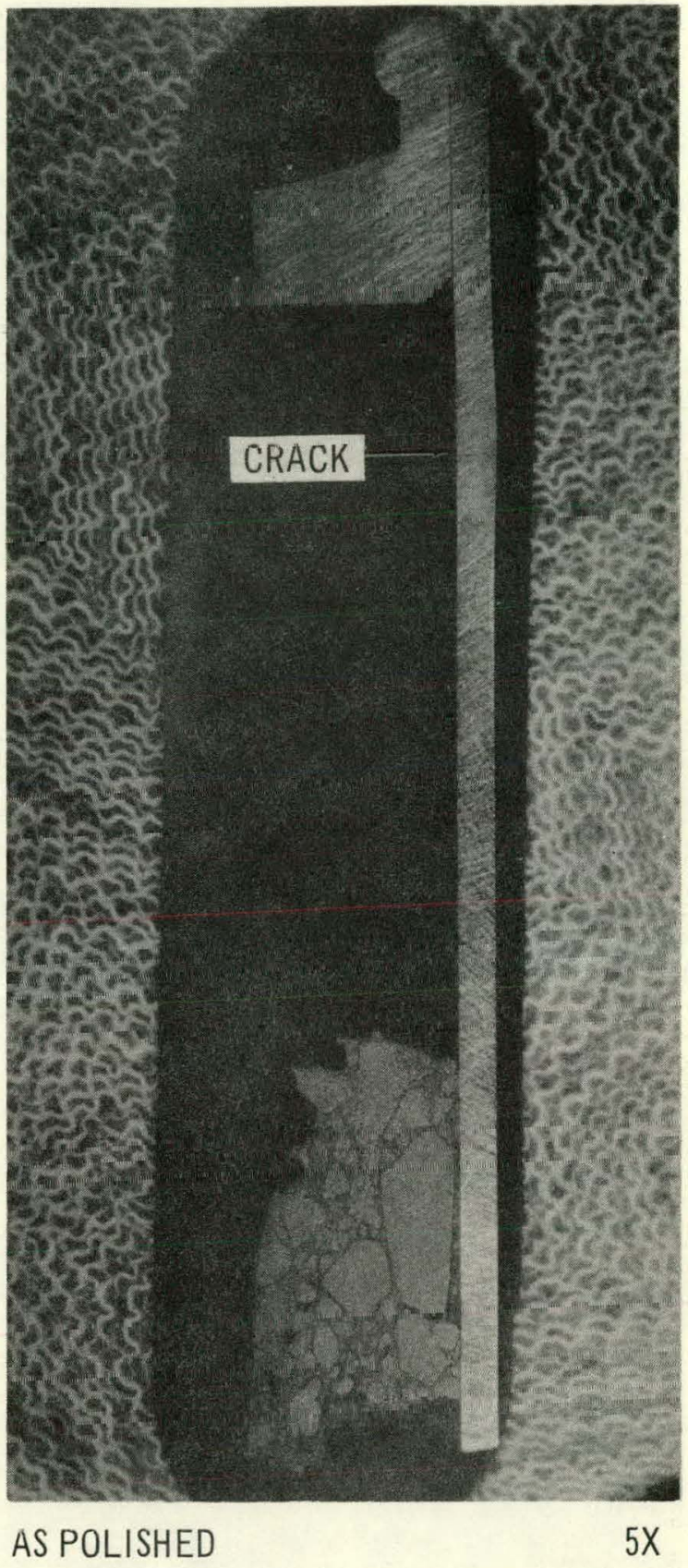

Figure 29B. Second Metallographic Plane of CR-163, Upper Failure 


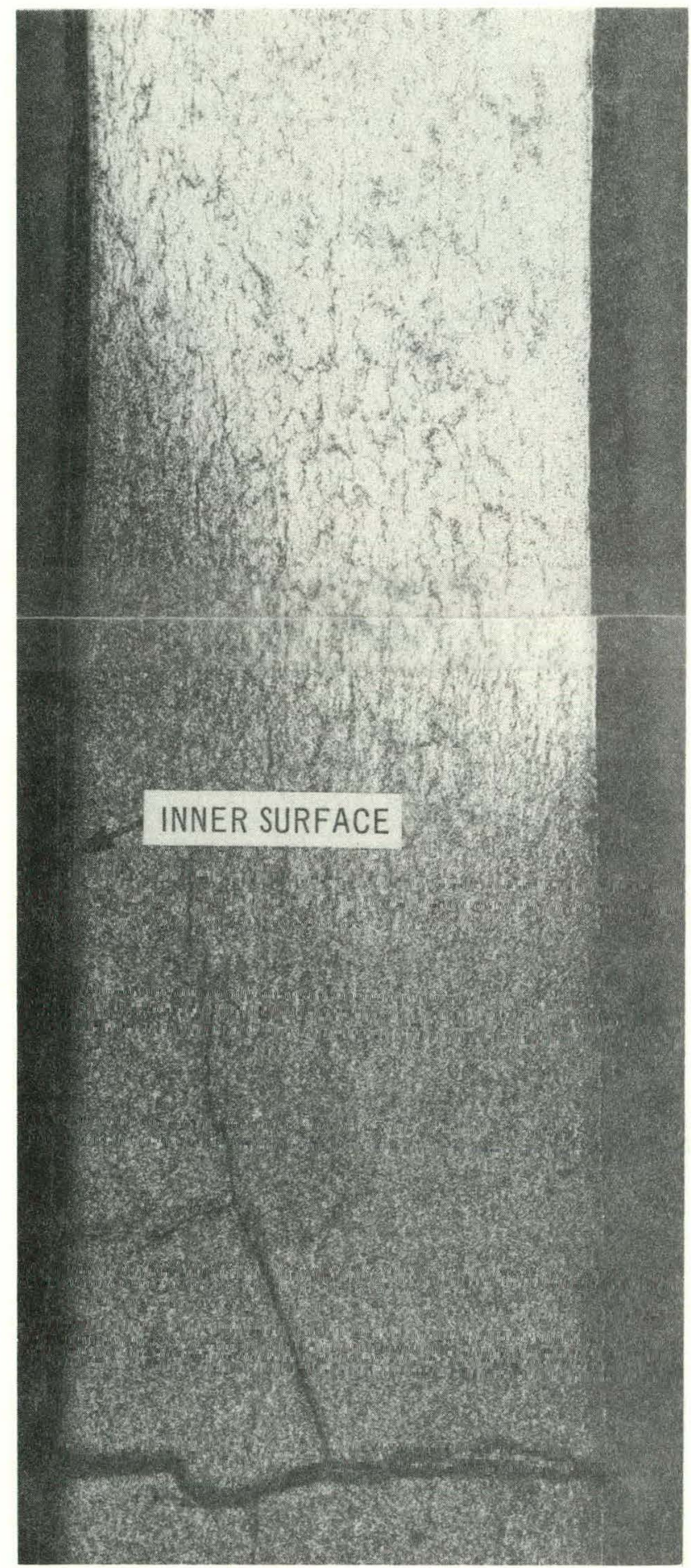

ETCHED
$75 X$
Figure 30. Crack and Cladding Hydride of Zircaloy-2 Clad Fuel Rod CR-163, Upper Failure

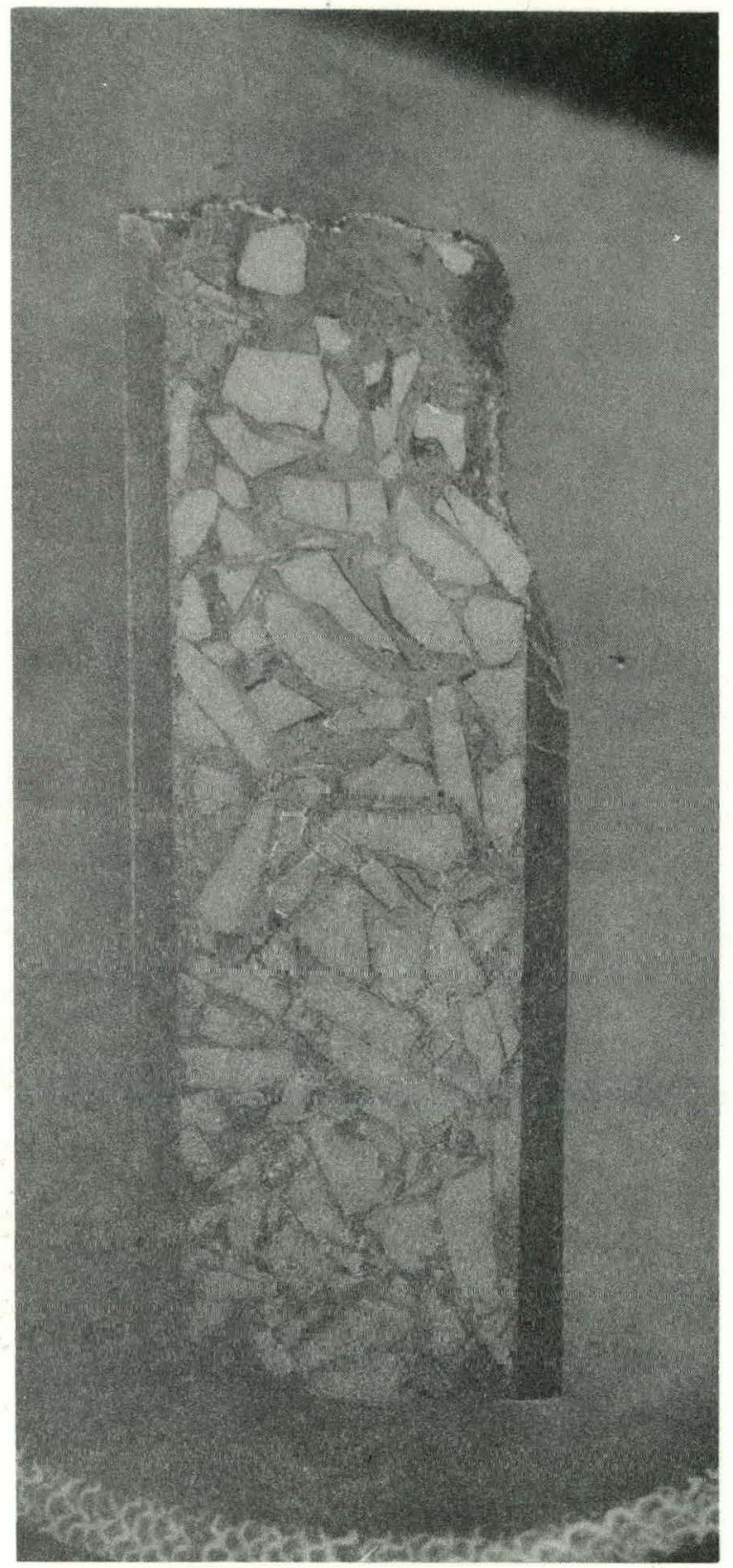

AS GROUND

$5 X$
Figure 31. Metallographic Plane of Zircaloy-2 Clad Fuel Rod CR-163, Lower Failure 


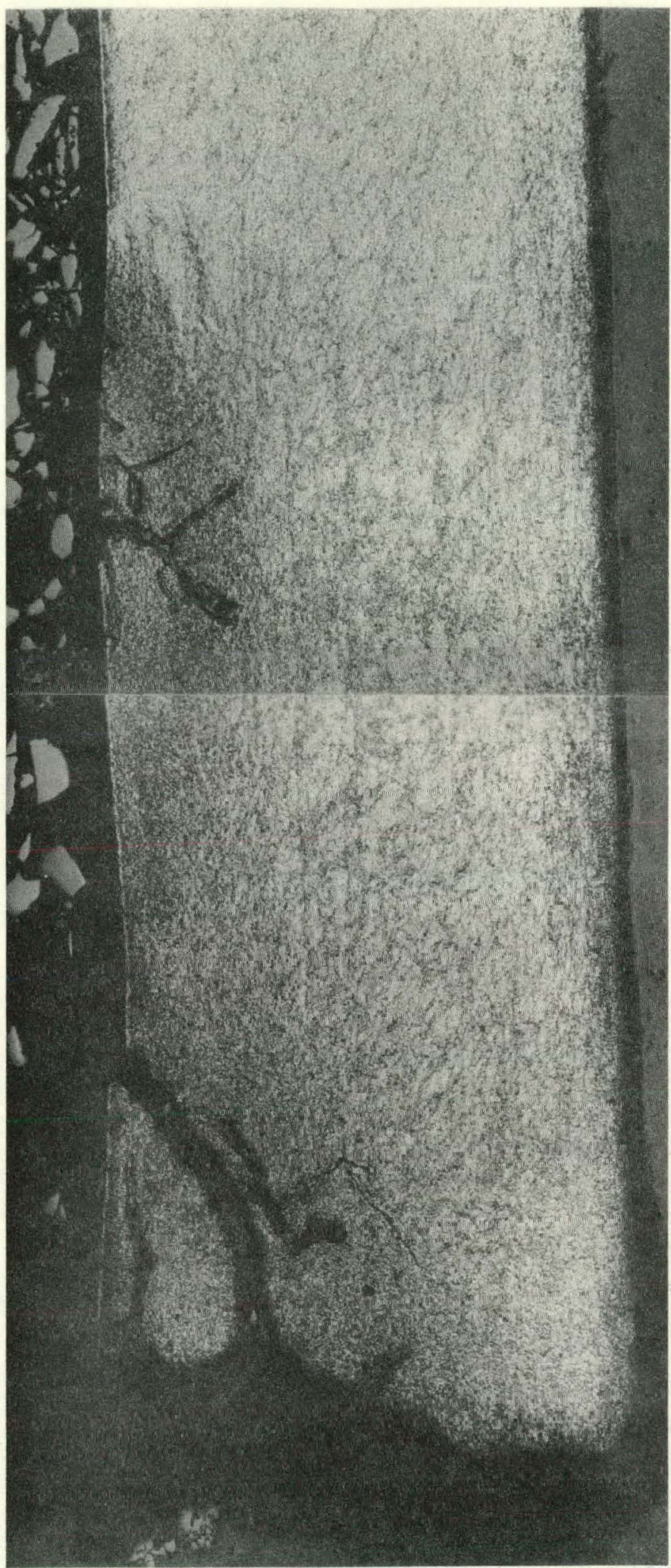

ETCHED
$75 X$

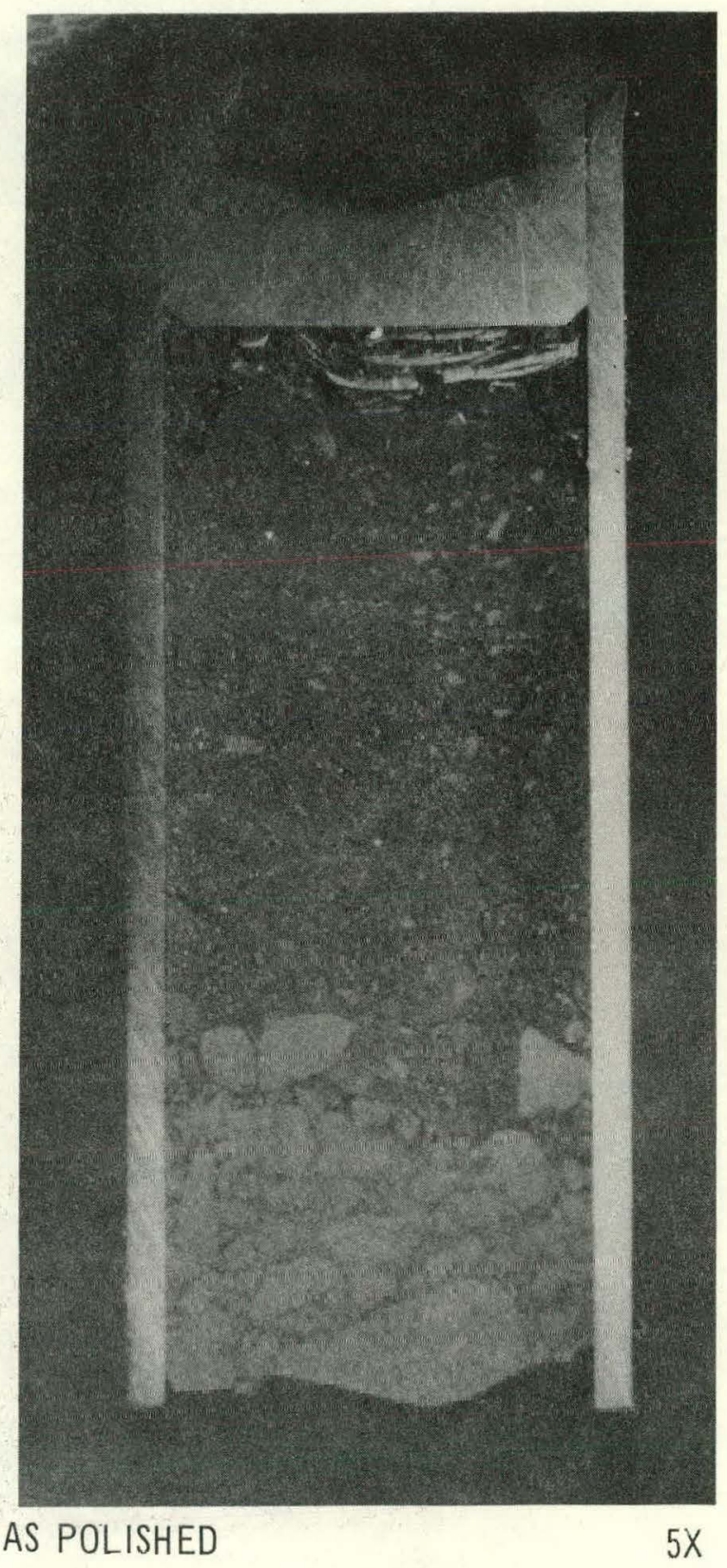

Figure 33. Metallographic Plane of Zircaloy-2 Clad

\author{
Fuel Rod CR-125, Upper End
}

Figure 32. Cracks and Cladding Hydrides of Zircaloy 2 Clad Fuel Rod CR-163 


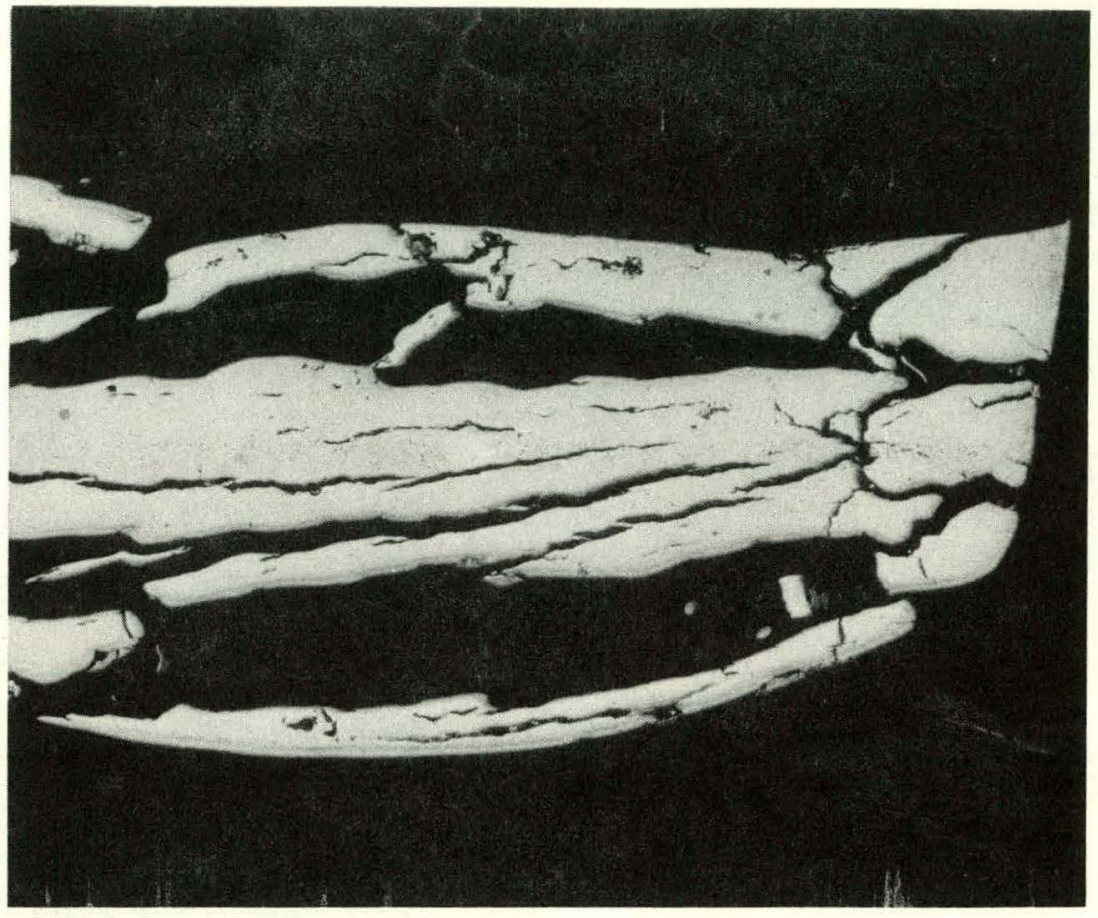

AS POLISHED

$100 X$

Figure 34. Fuel Rod CR-125 Separator Disc (as Polished)

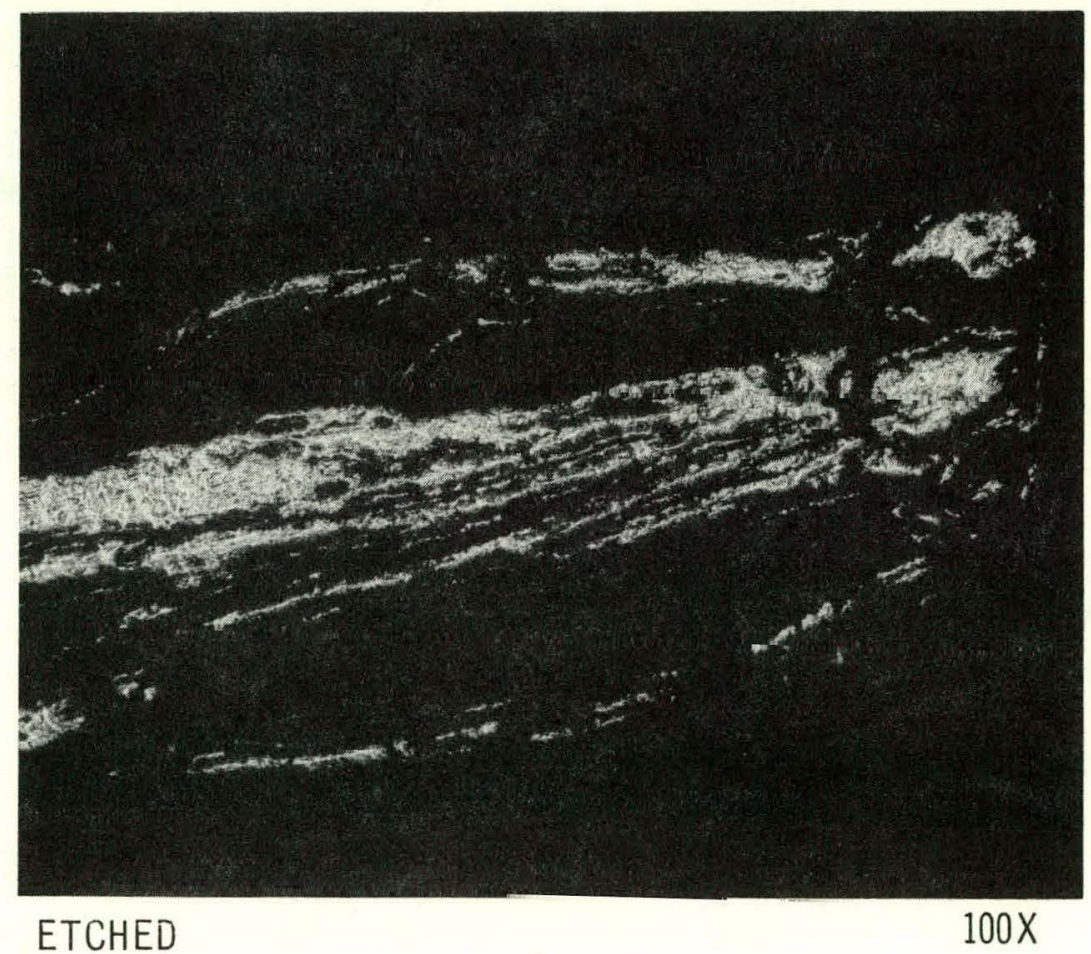

Figure 35. Fuel Rod CR-125 Separator Disc (Etched) 

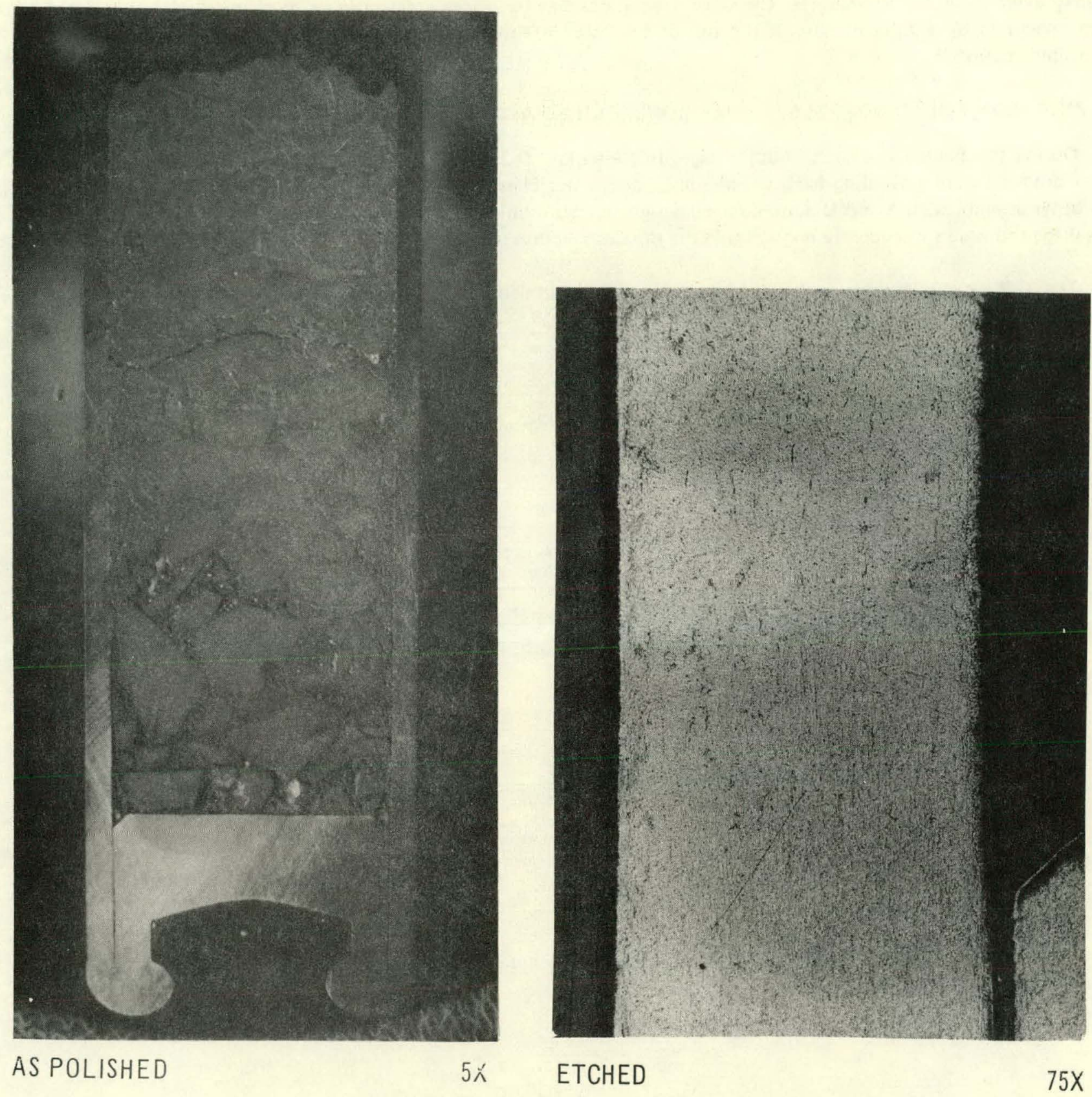

Figure 36. Metallographic Section of Fuel Rod CR-125, Lower End

Figure 37. Lower End Plug and Cladding of Fuel Kod C'R-125 


\subsubsection{Conclusions on Vipak Powder Fuel Rod Failures}

The observations on rods from bundle D-19, Zircaloy-2 clad Vipak powder are consistent with a hydrogenous impurity explanation for the failures. The D-19 rod failures may have been accelerated by mechanical stresses imposed on the cladding by a Zircaloy disc at the top of the fuel. Additional work is required to verify and identify the responsible impurities.

\subsection{ZIRCALOY-2 CLAD UO 2 PELLET FUEL RODS (FAILED ASSEMBLY D-2)}

During the February-March 1968, outage, fuel assembly D-2 was leak-checked. The leak check indicated that fission products were emanating from the assembly, and it was classed as failed. The exposure was 16,470 MWd/TU. Two other assemblies, D-1 and D-3, of identical design and with similar exposure, gave no indication of fission product gas leakage and were subsequently reinserted in the Big Rock Point reactor core.

The D-2 assembly was visually examined, using both underwater TV equipment and a periscope. No visual indication of failure was observed. 


\section{REFERENCES}

1. Fowler, W. D., Mechanical Design and Testing of Developmental Fuel for Consumers Big Rock Reactor, (GEAP-3851).

2. Holladay, R. L., High Power Density Development Project, Eleventh Quarterly Progress Report, October-December 1962, January 1963, (GEAP-4155).

3. Holladay, R. L., High Power Density Development Project, Sixteenth Quarterly Progress Report, January-March 1964, April 1964, (GEAP-4609).

4. Holland, L. K., High Power Density Development Project, Second Quarterly Progress Report, July-September 1960, October 1960, (GEAP-3579).

5. Fowler, W. D., and Lingafelter, J. W., Design and Fabrication of High Power Density Fuel Assemblies. for VBWR Irradiation Testing. November 1960, (GEAP-3609).

6. Lees, E. A., Fuel Element Fabrication by Swaging, (GEAP-3918).

7. DeHollander, W. R., Vibration Compaction of Uranium Dioxide, (GEAP-4032).

8. Ryer, C. M., Powder Fuel Processing by Two-Pass Swaging-Effect of Particle Size Distribution, (GEAP-3891).

9. Holladay, R. L., High Power Density Development Project, Tenth Quarterly Progress Report, July-September 1962, October 1962, (GEAP-4096).

10. Holland, L. K., High Power Density Development Project, Third Quarterly Progress Report, October-December 1960, January 1961, (GEAP-3632).

11. Hazel, V. E., et al., Fuel Irradiations in the ESADE-VBWR Nuclear Superheat Loop, March 1965, (GEAP-4775).

12. Spalaris, C. N., Incoloy-800 for Nuclear Fuel Sheaths (A Monograph), July 1964. (GEAP-4633).

13. Barth, N. H., and Haling, R. K., "Power Flattening Techniques Boost BWR Core Capabilities," Nucleonics 23 No. 5,72 (1965).

14. Holladay, R. L., High Power Density Development Project, Eighteenth Quarterly Progress Report, July-September 1964, October 1964, (GEAP-4741).

15. Kirby, R. F., et al., Fabrication of Fuel Cladding from Incoloy Alloy 800 (An Evaluation of Method), April 1964. (GEAP-4557).

16. Holladay, R. L., High Power Density Development Project, Seventeenth Quarterly Progress Report, April-June 1964, July 1964, (GEAP-4647).

17. Holladay, R. L., High Power Density Development Project, Twentieth Quarterly Progress Report, March-May 1965, June 1965, (GEAP-4894).

18. Howard, C. L., and Duncan, R. N., High Power Density Development Project, Twenty-Third and Twenty-Fourth Quarterly Progress Reports, December 1965-May 1966, June 1966, (GEAP-5126).

19. Howard, C. L., and Duncan, R. N., High Power Density Development Project, Twenty-Fifth and Twenty-Sixth Quarterly Progress Reports, June-November 1966, December 1966, (GEAP-5405).

20. Worthington, J. T., and Hazel, V. E., High Power Density Development Projects, Twenty-Seventh and Twonty Eighth Quorterly Proyitess Repurts, December 1966-May 1967, June 1967, (GEAP-5507).

21. Hazel, V. E., Post-Irradiation Examination of Zircaloy-2 and Incoloy-800 Clad Fuel Rods Irradiated to 7000 MWd/TU in the Consumers Big Rock Point Reactor, April 1968, (GEAP-5626).

22. Arlt, W. H., and Vandenberg, S. R., Fuel Failure Examinations in the High Power Density Program, September 1963, (GEAP-4360).

23. Holladay, R. L., High Power Density Development Project, Nineteenth Quarterly Report, October 1964 to February 1965, March 1965, (GEAP-4816).

24. Lees, E. A., Analysis of Fallure of Type-304 Stainless Steel Clad Swaged Powder Fuel Assembly, October 1963, (GEAP-4400).

25. Low, J. R., Wolff, U. E., and Cowden, W. C., Electron Microscopic Examination of Failed Stainless Steel Fuel Cladding-Dresden Advanced Fuel Assemblies PF-1 and PF-4, Rev. 1, March 1966, (APED-4242).

26. Pashos, T. J., Stainless Steel Failure Investigation Program, First Quarterly Progress Report, February 15, 1965-June 30, 1965, July 1965. (GEAP-4915).

27. Duncan, R. N.. Stainless Steel Failure Investigation Program, Fourth Quarterly Progress Report, January 1, 1966-March 31, 1966, July 1965, (GEAP-5150). . 
28. Duncan, R. N., Stainless Steel Failure Investigation Program, Fifth Quarterly Progress Report, April 1, 1966-June 30, 1966, August 1966, (GEAP-5194).

29. Freshley, M. D., and Flaherty, W. J., Ceramics Research and Development Operation and Quarterly, Report, January-March 1964, (HW-81600), pp.4.5-4.7.

30. Williamson, H. E., et al., AEC Fuel CVcle Program Examination of Zircalov Clad UO $\mathrm{O}_{2}$ Fuel Rods Operated in the VBWR to 10,000 MWd/TU, March 1965, (GEAP-4597).

31. Heavy Water Moderated Power Reactors Progress Report, April 1961, (DP-615). 


\section{APPENDIX. UNDERWATER PROFILOMETER}

The profilometer is a device that can continuously measure diametral variations along the length of an irradiated fuel rod under water.

The profilometer uses a pair of high-sensitivity linear differential transformers as position sensors. These transducers are mounted on a cross-head which can be moved axially over the length of the fuel rod. The fuel rod is positioned at each end in collets and can be rotated. The various combinations of relative transducer to fuel rod movements that can be obtained include longitudinal (axial trace), rotational (circumferential trace), or simultaneous axial and rotational movement (helical trace).

The outputs from the transducers are added together to give a precise measurement of deviations from a reference diameter standard used to zero the transducers.

The summed output signal is recorded on the $X$-axis of the $X-Y$ recorder. The $Y$-axis is voltage driven, using a linear potentiometer mechanically coupled to the axial drive mechanism of the profilometer. A time-basis option for the $\mathrm{Y}$-axis is also provided to enable recording circumferential profiles.

In addition, a dial gage has been included to enable the overall length of irradiated fuel rods to be measured to within 0.003 inch.

The Consumers pool profilometer was built to precisely measure dimensions of the irradiaten deyplnpmental fuol rod3. Selected fuel luds are. Pemoved trom various developmental assemblies, measured, and then reinserted into the assembly for continued irradiation.

Normally, profilometer traces are made of fuel rod diametral changes versus both length and angular position. From a $\Delta D$ versus $L$ plot, obtained by a straight axial traverse (no rotation of fuel rod), such phenomena as local wear or collapse, as well as circumferential ridging of cladding at $\mathrm{UO}_{2}$ pellet interfaces or at other discontinuities, can be measured.

A $\Delta D$ versus $L$ helical trace along the length of a fuel rod (i.e., made with the rod rotating) will indicate. maximum and minimum diameters and reveal longitudinal ridging or ovality of the fuel rod cladding.

A $\Delta D$ versus angular position trace obtained by rotating a fuel rod at a particular position along its length will indicate the number of lobes present if the fuel cladding has collapsed, or can be used to determine the degree of ovality of a fuel rod more precisely. To record $\Delta D$ versus angle traces, the recorder time base is used for the $Y$-axis.

A profilometer replaces mnre tedious, lece prccisc, measuriily mtihods such as micrometers or dial gages. The' profilometer takes continuous measurements over the entire fuel rod rather than at selected points, reducing the possibility of missing significant measurements which might otherwise be overlooked.

The major limitation of using a system that measures $\Delta D$ is the inability to distinguish a single cladding deformation from two diametrically opposed deformations. In addition, the interpretation of profile traces must take into account the geometry of the specimen-to-sensor contact. Two parallel rods (initially $1 / 4$-inch diameter, changed to 1/8-inch diameter) were used as the sensor contacts on the Consumers pool profilometer. The specimen-to-contact geometry sets the minimum size of a depression that can be measured and introduces some error if the radius of the depression or ridge being measured is less than the sensor contact radius. This iristrument should not be considered a surface finish profilometer; however, the maximum diameter of ridges and fuel rod ovality can be measured accurately.

It is possible to obtain profile traces that are reproducible to better than 0.0001 inch. Absolute diameter measurements can be made to better than 0.0002 inch, provided that an accurate standard is used to zero the transducers. However, to achieve this absolute accuracy it is necessary to minimize all factors that cause an apparent diameter change. These include: mechanical play, slippage at friction stops, and elastic deformation in the profilometer 
crosshead; wear of misalignment of the sensor contacts; ${ }^{*}$ and electrical-mechanical mismatch between the transducers when there is misalignment between the fuel rod and transducers (as. is possible with severely bowed fuel rods or eccentricity at the collets because of foreign material or misaligned end plugs or weld beads). In practice, it is necessary to measure a dimensional standard before and after each fuel rod to achieve maximum accuracy. This is not'practical in view of other work requirements at a fuel outage, so usually four to eight fuel rods are measured between standard checks. This gives an absolute accuracy, typically 0.0005 to 0.001 inch.

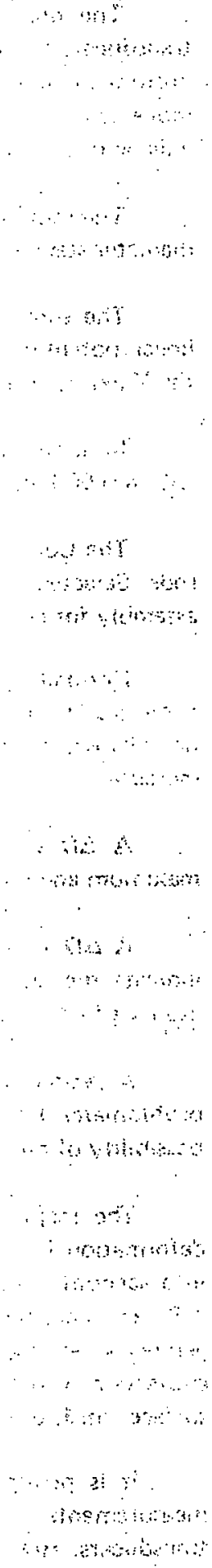

- Wear of each transducer tip (tungsten carbide) is typically 0.0002 to 0.0005 inch per 10 to 20 deposittaden fuel rods meäsured. 


\section{DISTRIBUTION}

U. S. Atomic Energy Commission Division of Reactor Development Washington 25, D. C.

Attn: Chief, Water Project

U. S. Atomic Energy Commission Division of Reactor Development Washington 25, D. C.

Attn: Chief, Water Systems Branch Army Reactors

U. S. Atomic Energy Commission Division of Reactor Development Washington 25, D. C.

Attn: Chief, Reactor Engineering Branch Naval Reactors

U. S. Atomic Energy Commission Division of Reactor Development Washington 25, D. C.

Attn: Chief Thermal Reactors Safety Branch

U. S. Atomic Criteryy Cummissión Division of Reactor Development Washington 25, D. C.

Attn: Chief, Fuels and Materials Branch

U. S. Atomic Energy Commission Chicago Operations Office 9800 South Cass Avenue Argonne, lllinois

Attn: Director, Reactor Engineering Division

U. S. Atomic Energy Commission San Francisco Operations Office 2111 Bancroft Way Berkeley, California

Attn: Director, Contracts Division

U.S. Atomic Energy Commission

RDT

Sr. Site Representative-GE

310 DeGuigne Drive

Sunnyvale, California 94086

Attn: R. H. Ball

Library

Atomic Energy Division

Allis-Chalmers Manufacturing Co.

6935 Arlington Road

Bethesda, Maryland 20014
3 Babcock and Wilcox Company

Atomic Energy Division

Lynchburg, Virginia

Attn: J.F. Mumm

1 Mr. J. M. West

Combustion Engineering Inc.

Nuclear Division

1000 Prospect Hill Road

Windsor, Conn.

1 Westinghouse Electric Corporation

1

Bettis Atomic Power Laboratory

P. O. Box 79

West Mifflin, Pennsylvania

Attn: W. E. Johnson

1 Consumers Power Company

212 W. Michigan Avenue

10

Jackson, Michigan

Attn: R. L. Haueter

Northern States Power Company

1414 Nicollet Avenue

Minneapolis, Minnesota 55401

Attn: Mr. E. C. Ward

Argonne National Laboratory

P. O. Box 2528

2 Idaho Falls, Idaho

Attn: Meyer Novick

Argonne National Laboratory

Library Services Department

Report Section - BIdg. 203-Rm-Ce-125

9700 South Cass Avenue

1 Argonne, Illinois

U. S. Atomic Energy Commission

3

Technical Information Center

P. O. Box 62

Oak Ridge, Tennessee

1 Attn: R. L. Metter

Nuclear Utilities Services, Inc

1730 M Street NW

Washington, D. C.

Attn: J. C. Scarborough

1 R. E. Shimshak

Dairyland Power Cooperative

La Crosse Boiling Water Reactor

P. O. Box 135

Genoa, Wisconsin 